\title{
Molybdenum(VI) Bis-Imido Complexes of Dipyrromethene Ligands
}

Jan Locher, ${ }^{a}$ Fabian A. Watt, ${ }^{a}$ Adam G. Neuba, ${ }^{a}$ Roland Schoch, ${ }^{a}$ Dominik Munz, ${ }^{b, c^{*}}$ and Stephan Hohloch, ${ }^{\text {d* }}$

Supporting Information

\section{Table of contents}

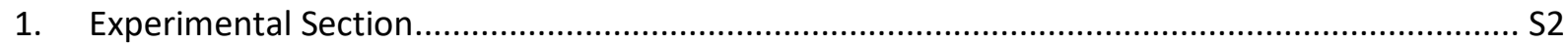

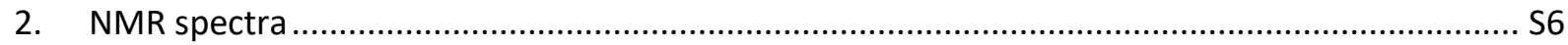

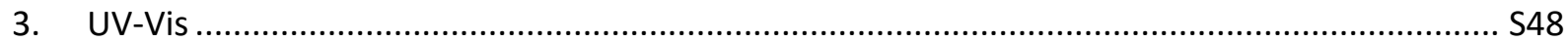

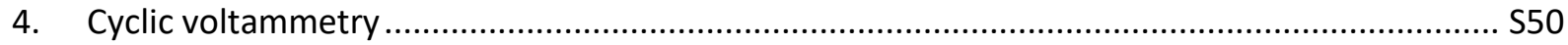

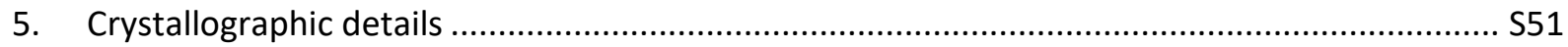

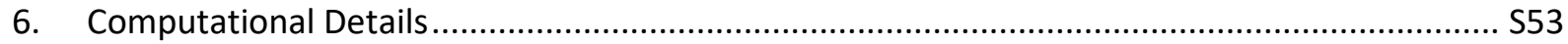

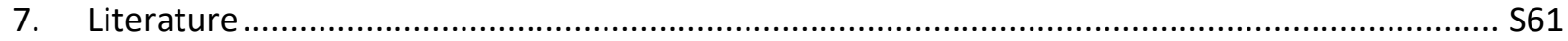

aPaderborn University, Faculty of Science, Department of Chemistry, Warburger Straße 100, 33098 Paderborn, Germany.

bUniversity of the Saarland, Inorganic Chemistry, 66123 Saarbrücken, Germany.

CFriedrich-Alexander-Universität Erlangen-Nürnberg, Egerlandstrasse1, 91058 Erlangen, Germany.

dUniversity of Innsbruck, Faculty of Chemistry and Pharmacy, Institute of General, Inorganic and Theoretical Chemistry, Innrain 80-82, 6020 Innsbruck, Austria

E-Mail: Stephan.Hohloch@uibk.ac.at 


\section{Experimental Section}

\section{General remarks}

If not otherwise mentioned, all transformations were carried out in an argon filled glovebox. Solvents were dried by a MBraun SPS system and stored over activated molecular sieves ( $3 \AA$ ) for at least one day. Deuterated $\mathrm{C}_{6} \mathrm{D}_{6}$ was dried over sodium/benzophenone and $\mathrm{CDCl}_{3}$ and $\mathrm{CD}_{2} \mathrm{Cl}_{2}$ over calcium hydride, followed by vacuum transfer and three freeze-pump-thaw cycles prior to use. Starting materials $\mathrm{HL}^{1},{ }^{1,2} \mathbf{H L}^{2},{ }^{3}$ (DME) $\mathrm{MoO}_{2} \mathrm{Cl}_{2},{ }^{4}(\mathrm{DME}) \mathrm{Mo}\left(\mathrm{N}^{t} \mathrm{Bu}_{2}\right)_{2} \mathrm{Cl}_{2}{ }^{5}$ $\mathrm{KC}_{8}{ }^{6}$ thiantrenium hexafluorophosphate ${ }^{7}$ and $\mathrm{Ag}\left[\mathrm{Al}(\mathrm{pftb})_{4}\right]^{8}$ were synthesized following literature known procedures. KHMDS, AgOTf and $\mathrm{NOPF}_{6}$ were used as received without further purification. $\mathrm{LiCH}_{2} \mathrm{SiMe}_{3}$ was purchased as a $1 \mathrm{M}$ solution in $n$-pentane and the $n$-pentane was evaporated at room temperature to give $\mathrm{LiCH}_{2} \mathrm{SiMe}_{3}$ as a colorless, pyrophoric powder. NMR spectra were collected at $303 \mathrm{~K}$ on a Bruker AV-500 or an Ascent 700 spectrometer using J-Young NMR tubes. ${ }^{1} \mathrm{H},{ }^{13} \mathrm{C},{ }^{15} \mathrm{~N},{ }^{19} \mathrm{~F}$ and ${ }^{29} \mathrm{Si} N \mathrm{NMR}$ chemical shifts $(\delta)$ are reported in ppm. ${ }^{1} \mathrm{H}$ and ${ }^{13} \mathrm{C}$ chemical shifts were calibrated to residual solvent peaks. ${ }^{15} \mathrm{~N}$ chemical shifts were referenced to liquid ammonia as an external standard. ${ }^{15} \mathrm{~N}$ data were recorded by ${ }^{1} \mathrm{H}-{ }^{15} \mathrm{~N}$ HMBC NMR spectra utilizing the polarization transfer from the ${ }^{1} \mathrm{H}$ nuclei. ${ }^{19} \mathrm{~F}$ chemical shifts were referenced to $\mathrm{CFCl}_{3}$ as an external standard. ${ }^{29} \mathrm{Si}$ chemical shifts were referenced to TMS $\left(\mathrm{Si}\left(\mathrm{CH}_{3}\right)_{4}\right)$ as an external standard. Elemental analyses were performed using an Elementar vario microcube instrument. UV-Vis data were collected using a Cary 50 scan instrument. Cyclic voltammetry was performed at room temperature using the PAR101 potentiostat by Metrohm in dichloromethane / $\left.0.1 \mathrm{M}^{2} \mathrm{NBu}_{4}\right] \mathrm{PF}_{6}$ electrolyte (analyte conc. $=0.001 \mathrm{~mol} \cdot \mathrm{L}^{-1}$ ) with the following three electrode arrangement: Pt working electrode $(1$ $\mathrm{mm}$ diameter), $\mathrm{Ag}$ wire as pseudo-reference and $\mathrm{Pt}$ wire counter electrode. Ferrocene was added as internal standard after the measurements and all potentials are referenced relative to the $\mathrm{Fc} /[\mathrm{Fc}]^{+}$redox couple.

\section{Computational details}

The computations were performed with ORCA, revision 4.0.1, ${ }^{9}$ at the PBE0-D3BJ/def2-TZVPP//PBEO-D3BJ/def2-SVP level of theory. ${ }^{10}$ The def2-ECP and the def2-TZVP valence basis sets were used for molybdenum in the geometry optimization. ${ }^{11}$ The optimized structures were verified as true minima by the absence of imaginary eigenvalues in the vibrational frequency analyses. The RIJCOSX approximation with the related auxiliary basis set def2/J was used to speed up the calculations. ${ }^{12}$ Tighter than default convergence criteria (tightopt, tightscf) and finer than default grid values (grid6, nofinalgrid, gridx6) were applied. The computed molecular orbitals were rendered with IBOView. ${ }^{13}$ Calculations with the BP86, M06 or TPSSh functionals led to comparable results (Fig. S48). The TDDFT calculations for $\mathbf{1}^{\text {cat }}$ were performed at the PBEO-D3BJ/def2-TZVPP//PBEO-D3BJ/def2-SVP level of theory (grid6, nofinalgrid, gridx6) with 50 roots using the Tamm-Dancoff approximation (cf. Fig. S52, S53). For the state-averaged CASSCF calculations for $\mathbf{1}^{\text {cat }}$, the "full" geometry of $\mathbf{1}^{\text {cat }}$ was investigated as well as a model with truncated mesityl substitutents (using methyl substituents for the structural parameters as obtained from DFT with the positions of the hydrogen atoms optimized). However, in agreement with the DFT calculations, interaction with the mesityl groups seems important and also large shifts of other transitions (as high as $0.9 \mathrm{eV}$ ) were observed for truncated vs. the non-truncated system. Hence, only the results with the non-truncated system are reported. An active space of 11 electrons in 14 orbitals was chosen. Here, 4 bonding and 4 antibonding orbitals related to the two flanking mesityl substituents of the DPM ligand, 1 bonding and 1 antibonding orbital relating to the $\pi$-system of the DPM ligand, 1 bonding and 1 antibonding imido $\pi$-type orbital and 2 further unoccupied orbitals associated mainly with the metal's d-orbitals / imido ligands (cf. Fig. S54). An enlargement of the active space (further d-orbitals, bridgehead mesityl group) might improve the results, but seems unfeasible in sight of tremendous computational demand. In this context, note the decent fit between the TD-DFT and CASSCF calculated transitions. The energies of the transitions were corrected by second order perturbation theory NEVPT2 (tightscf, nofrozencore, def2-TZVPP basis set, def2/J auxiliary basis set, RIJCOSX, 10 doublet states, 6 quartet states).

\section{X-ray crystallography}

Single crystals for $\mathrm{X}$-ray diffraction experiments were measured at the analytical facility of the Paderborn University using a Bruker Smart AXS or a Bruker D8 Venture instrument. All crystals were kept at 130(2) K throughout data collection. Data collection was performed using either the APEXIII or the Smart software package. Data refinement and reduction were performed with Bruker Saint (V8.34A). All structures were solved with SHELXT ${ }^{14}$ and refined using the OLEX 2 software package. ${ }^{15}$ All non-hydrogen atoms were refined anisotropically, and hydrogen atoms were included at the geometrically calculated positions and refined using a riding model. SQUEEZE application was used for heavily disordered solvents if necessary. ${ }^{16}$ CCDC 1962501 - 1962508 contain the CIF-Files for complexes 1 7 respectively. For further crystallographic details, please check Tables S1 and S2 in the Supporting Information. 
Ligand precursor H(DPMMes) (1 eq., $5.02 \mathrm{mmol}, 2.5 \mathrm{~g}$ ) was suspended in $n$-pentane $(50 \mathrm{~mL})$, giving an orange slurry, to which was added KHMDS (1.05 eq., $5.27 \mathrm{mmol}, 1.05 \mathrm{~g}$ ) in small portions. During the addition of KHMDS the color of the suspension changed from orange to pink with a pink-orange precipitate. The reaction mixture was stirred overnight at room temperature. Then, the slurry was filtered through a medium porosity frit and the pink-orange solid washed with minimal amounts of cold $\left(-40^{\circ} \mathrm{C}\right) n$-pentane, giving the desired potassium salt of the DPM ligand as a fine pink-orange powder in excellent yields of $94 \%$ (4.97 mmol, $2.54 \mathrm{~g}) .{ }^{1} \mathrm{H} \mathrm{NMR}\left(\mathrm{C}_{6} \mathrm{D}_{6}, 303 \mathrm{~K}, 700 \mathrm{MHz}\right): \delta(\mathrm{ppm})$ $=6.98(\mathrm{~d}, J=3.8 \mathrm{~Hz}, 2 \mathrm{H}$, pyrrole- $H), 6.92(\mathrm{~s}, 2 \mathrm{H}$, Mes- $H), 6.82(\mathrm{~s}, 4 \mathrm{H}$, Mes- $H), 6.45(\mathrm{~d}, J=3.8 \mathrm{~Hz}, 2 \mathrm{H}$, pyrrole- $H), 2.51$ (s, 6H, Mes- $\left.\mathrm{CH}_{3}\right), 2.29\left(\mathrm{~s}, 12 \mathrm{H}\right.$, Mes- $\left.\mathrm{CH}_{3}\right), 2.27\left(\mathrm{~s}, 3 \mathrm{H}\right.$, Mes- $\left.\mathrm{CH}_{3}\right), 2.23\left(\mathrm{~s}, 6 \mathrm{H}, \mathrm{Mes}-\mathrm{CH}_{3}\right) ;{ }^{13} \mathrm{C}\left\{{ }^{1} \mathrm{H}\right\} \mathrm{NMR}\left(\mathrm{C}_{6} \mathrm{D}_{6}, 303 \mathrm{~K}\right.$, $125 \mathrm{MHz}$ ): $\delta(\mathrm{ppm})=157.7$ (Aryl-C), 148.1 (Aryl-C), 142.2 (Aryl-C), 140.4 (Aryl-C), 137.7 (Aryl-C), 137.2 (Aryl-C), 137.0 (Aryl-C), 135.9 (Aryl-C), 135.8 (Aryl-C), 130.8 (pyrrole- $\mathrm{CH}$ ), 128.8 (Mes-CH) 127.8 (Mes- $\mathrm{CH}$ ) 117.2 (pyrrole-CH), 21.3 (Mes- $\left.\mathrm{CH}_{3}\right), 21.1$ (Mes- $\left.\mathrm{CH}_{3}\right), 21.0\left(\right.$ Mes- $\left.\mathrm{CH}_{3}\right), 20.7\left(\mathrm{Mes}-\mathrm{CH}_{3}\right) ;{ }^{15} \mathrm{~N}$ NMR $\left(\mathrm{C}_{6} \mathrm{D}_{6}, 303 \mathrm{~K}, 176 \mathrm{MHz}\right): \delta(\mathrm{ppm})=278.4$ (pyrrole- $N$ ); elemental analysis (\%) calcd for $\mathrm{C}_{36} \mathrm{H}_{37} \mathrm{~N}_{2} \mathrm{~K}$ : C $80.55 \mathrm{H} 6.95 \mathrm{~N} \mathrm{5.22,} \mathrm{found} \mathrm{C} 80.32 \mathrm{H} 7.20 \mathrm{~N} 4.89$.

\section{$K\left(D P M^{t B u}\right)\left(K L^{2}\right)$}

Ligand precursor H(DMPtBu) (1 eq., $3 \mathrm{mmol}, 1.12 \mathrm{~g}$ ) was suspended in $n$-pentane $(50 \mathrm{~mL})$ and KHMDS (1.05 eq, $3.15 \mathrm{mmol}, 630 \mathrm{mg}$ ) was added in small portions as a solid over of period of $25 \mathrm{~min}$. The resulting orange suspension was stirred overnight and the orange solid was collected by filtration and washed with $20 \mathrm{~mL}$ of cold $\left(-40^{\circ} \mathrm{C}\right) n$ pentane to yield the desired potassium salt of the DPM ligand as a fine orange powder in a yield of $88 \%(2.64 \mathrm{mmol}$, $1.09 \mathrm{~g}) .{ }^{1} \mathrm{H}$ NMR $\left(\mathrm{Py}_{-} \mathrm{d}_{5}, 303 \mathrm{~K}, 700 \mathrm{MHz}\right): \delta(\mathrm{ppm})=6.90(\mathrm{~s}, 2 \mathrm{H}, \mathrm{Mes}-\mathrm{CH}), 6.81(\mathrm{~s}, 2 \mathrm{H}$, broad, pyrrole- $H), 6.60(\mathrm{~s}, 2 \mathrm{H}$, pyrrole- $H$ ), $2.32\left(\mathrm{~s}, 3 \mathrm{H}\right.$, Mes- $\left.\mathrm{CH}_{3}\right), 2.19\left(\mathrm{~s}, 6 \mathrm{H}, \mathrm{Mes}-\mathrm{CH}_{3}\right), 1.48\left(\mathrm{~s}, 18 \mathrm{H}\right.$, pyrrole- $\left.\mathrm{C}\left(\mathrm{CH}_{3}\right)_{3}\right) ;{ }^{13} \mathrm{C}\left\{{ }^{1} \mathrm{H}\right\}$ NMR $\left(\mathrm{Py}-\mathrm{d}_{5}, 303 \mathrm{~K}\right.$, $125 \mathrm{MHz}): \delta(\mathrm{ppm})=170.1($ Aryl-C), $145.4($ Aryl-C), $141.0($ Aryl-C), 139.7 (Aryl-C), 136.8 (Aryl-C), 130.7 (pyrrole- $\mathrm{CH})$, 127.4 (Mes- $\mathrm{CH}$ ), 112.4 (pyrrole- $\mathrm{CH}$ ), 34.3 (pyrrole- $\left.\mathrm{C}\left(\mathrm{CH}_{3}\right)_{3}\right), 30.9$ (pyrrole- $\left.\mathrm{C}\left(\mathrm{CH}_{3}\right)_{3}\right), 20.9\left(\mathrm{Mes}-\mathrm{CH}_{3}\right), 20.2\left(\mathrm{Mes}-\mathrm{CH}_{3}\right)$. No ${ }^{15} \mathrm{~N}$ NMR data could be obtained due to the low solubility of the potassium salt. Elemental analysis (\%) calcd for $\mathrm{C}_{26} \mathrm{H}_{33} \mathrm{~N}_{2} \mathrm{~K}$ : C 75.68 H 8.06 N 6.79, found C 75.34 H 7.97 N 6.46.

\section{(DPMMes) Mo(NtBu$)_{2} \mathrm{Cl}(1)$}

Solid KL${ }^{1}$ (1 eq., $2 \mathrm{mmol}, 1.07 \mathrm{~g}$ ) and solid (DME)Mo( ${ }^{t} \mathrm{Bu}_{2} \mathrm{Cl}_{2}$ (1 eq., $2 \mathrm{mmol}, 800 \mathrm{mg}$ ) were mixed and cooled to $40{ }^{\circ} \mathrm{C}$. To this mixture $-40^{\circ} \mathrm{C}$ cold diethyl ether $(50 \mathrm{~mL})$ was added and the reaction mixture stirred overnight, while warming to room temperature. The solution turned dark pink concomitant with the formation of an orange-white precipitate. The crude reaction mixture was filtered and the pink solution was evaporated to dryness. Lyophilizing the resulting pink solids from frozen benzene resulted in the formation of the desired molybdenum complex as a fluffy red-pink solid in a yield of $84 \%(1.68 \mathrm{mmol}, 1.295 \mathrm{~g})$. X-ray quality crystals were grown from a concentrated solution of 1 in $n$-pentane over the course of one hour. ${ }^{1} \mathrm{H} N M R\left(\mathrm{C}_{6} \mathrm{D}_{6}, 303 \mathrm{~K}, 700 \mathrm{MHz}\right): \delta(\mathrm{ppm})=6.89(\mathrm{~s}, 1 \mathrm{H}, \mathrm{Mes}-$ $\mathrm{CH}), 6.85(\mathrm{~s}, 1 \mathrm{H}$, Mes-CH), $6.85(\mathrm{~s}, 1 \mathrm{H}$, Mes-CH), $6.82(\mathrm{~s}, 1 \mathrm{H}, \mathrm{Mes}-\mathrm{CH}), 6.81(\mathrm{~s}, 2 \mathrm{H}$, Mes-CH), $6.74(\mathrm{~d}, J=4.1 \mathrm{~Hz}, 1 \mathrm{H}$, pyrrole- $H$ ), $6.71(\mathrm{~d}, J=4.1 \mathrm{~Hz}, 1 \mathrm{H}$, pyrrole- $H), 6.42(\mathrm{~d}, J=4.1 \mathrm{~Hz}, 1 \mathrm{H}$, pyrrole- $H), 6.10(\mathrm{~d}, J=4.1 \mathrm{~Hz}, 1 \mathrm{H}$, pyrrole- $H$ ), $2.77\left(\mathrm{~s}, 3 \mathrm{H}, \mathrm{Mes}-\mathrm{CH}_{3}\right), 2.64\left(\mathrm{~s}, 3 \mathrm{H}, \mathrm{Mes}-\mathrm{CH}_{3}\right), 2.51\left(\mathrm{~s}, 3 \mathrm{H}, \mathrm{Mes}-\mathrm{CH}_{3}\right), 2.36\left(\mathrm{~s}, 3 \mathrm{H}, \mathrm{Mes}-\mathrm{CH}_{3}\right), 2.31\left(\mathrm{~s}, 3 \mathrm{H}, \mathrm{Mes}-\mathrm{CH}_{3}\right)$, $2.223\left(\mathrm{~s}, 3 \mathrm{H}, \mathrm{Mes}-\mathrm{CH}_{3}\right) 2.19\left(\mathrm{~s}, 3 \mathrm{H}, \mathrm{Mes}-\mathrm{CH}_{3}\right), 2.17\left(\mathrm{~s}, 3 \mathrm{H}, \mathrm{Mes}-\mathrm{CH}_{3}\right), 2.10\left(\mathrm{~s}, 3 \mathrm{H}, \mathrm{Mes}-\mathrm{CH}_{3}\right), 1.35(\mathrm{~s}, 9 \mathrm{H}, \mathrm{Mo}=\mathrm{N}-$ $\left.\mathrm{C}\left(\mathrm{CH}_{3}\right)_{3}\right), 0.91\left(\mathrm{~s}, 9 \mathrm{H}, \mathrm{Mo}=\mathrm{N}-\mathrm{C}\left(\mathrm{CH}_{3}\right)_{3}\right) ;{ }^{13} \mathrm{C}\left\{{ }^{1} \mathrm{H}\right\} \operatorname{NMR}\left(\mathrm{C}_{6} \mathrm{D}_{6}, 303 \mathrm{~K}, 125 \mathrm{MHz}\right): \delta(\mathrm{ppm})=168.4$ (Aryl-C), $164.9($ Aryl-C), 145.8 (Aryl-C), 140.8 (Aryl-C), 140.7 (Aryl-C), 138.3 (Aryl-C), 138.0 (Aryl-C), 137.9 (Aryl-C), 137.2 (Aryl-C), 136.9 (ArylC), 135.0 (Aryl-C), 134.7 (Aryl-C), 134.3 (Aryl-C), 132.6 (pyrrole-CH), 131.6 (pyrrole-CH), 129.7 (Mes- $C H$ ), 129.4 (Mes$\mathrm{CH}), 128.8$ (Mes- $\mathrm{CH}), 124.5$ (pyrrole- $\mathrm{CH}), 119.7$ (pyrrole- $\mathrm{CH}), 73.3\left(\mathrm{Mo}=\mathrm{N}-\mathrm{C}\left(\mathrm{CH}_{3}\right)_{3}\right), 71.6\left(\mathrm{Mo}=\mathrm{N}-\mathrm{C}\left(\mathrm{CH}_{3}\right)_{3}\right), 31.0$ $\left(\mathrm{Mo}=\mathrm{N}-\mathrm{C}\left(\mathrm{CH}_{3}\right)_{3}\right), 29.9\left(\mathrm{Mo}=\mathrm{N}-\mathrm{C}\left(\mathrm{CH}_{3}\right)_{3}\right), 23.4\left(\mathrm{Mes}-\mathrm{CH}_{3}\right), 23.1\left(\mathrm{Mes}-\mathrm{CH}_{3}\right), 22.9\left(\mathrm{Mes}-\mathrm{CH}_{3}\right), 21.5\left(\mathrm{Mes}-\mathrm{CH}_{3}\right), 21.5(\mathrm{Mes}-$ $\left.\mathrm{CH}_{3}\right), 21.4$ (Mes- $\left.\mathrm{CH}_{3}\right), 21.3\left(\right.$ Mes- $\left.\mathrm{CH}_{3}\right), 19.9\left(\right.$ Mes- $\left.\mathrm{CH}_{3}\right) ;{ }^{15} \mathrm{~N} \mathrm{NMR}\left(\mathrm{C}_{6} \mathrm{D}_{6}, 303 \mathrm{~K}, 176 \mathrm{MHz}\right): \delta(\mathrm{ppm})=242.0$ (pyrrole$N), 209.8($ pyrrole- $N), 119.9,\left(\mathrm{Mo}=N-\mathrm{C}\left(\mathrm{CH}_{3}\right)_{3}\right), 100.3\left(\mathrm{Mo}=\mathrm{N}-\mathrm{C}\left(\mathrm{CH}_{3}\right)_{3}\right)$; elemental analysis (\%) calcd for $\mathrm{C}_{44} \mathrm{H}_{55} \mathrm{~N}_{4} \mathrm{ClMo}$ : C 68.51 H 7.19 N 7.26, found C 68.72 H 7.25 N 6.98 .

\section{(DPMMes) $\mathrm{Mo}(\mathrm{NMes})_{2} \mathrm{Cl}(2)$}

Solid $\mathrm{KL}^{1}$ (1 eq, $0.3 \mathrm{mmol}, 161 \mathrm{mg}$ ) and solid (DME)Mo(NMes) ${ }_{2} \mathrm{Cl}_{2}$ (1 eq, $0.3 \mathrm{mmol}, 157 \mathrm{mg}$ ) were mixed and cooled to $-40{ }^{\circ} \mathrm{C}$. To this mixture $-40{ }^{\circ} \mathrm{C}$ cold diethyl ether $(1 \mathrm{~mL})$ was added and the reaction mixture stirred overnight, while warming to room temperature. The dark red reaction mixture was filtered to remove solid potassium chloride and was evaporated to dryness. The red solid was then again dissolved in $n$-pentane $(15 \mathrm{~mL})$, filtered, concentrated to $1 \mathrm{~mL}$ and stored at room temperature overnight. This resulted in the deposition of dark red crystals of $\mathrm{X}$-ray quality in a yield of $81 \%(0.243 \mathrm{mmol}, 217 \mathrm{mg}) .{ }^{1} \mathrm{H} \mathrm{NMR}\left(\mathrm{C}_{6} \mathrm{D}_{6}, 303 \mathrm{~K}, 700 \mathrm{MHz}\right): \delta(\mathrm{ppm})=6.83(\mathrm{~m}, 3 \mathrm{H}, \mathrm{Mes}-\mathrm{CH}$ and pyrrole- $H$ ), $6.80(\mathrm{~d}, J=3.8 \mathrm{~Hz}, 1 \mathrm{H}$, pyrrole- $H), 6.74(\mathrm{~s}, 2 \mathrm{H}, \mathrm{Mes}-\mathrm{CH}), 6.49(\mathrm{~s}, 2 \mathrm{H}, \mathrm{Mes}-\mathrm{CH}), 6.48(\mathrm{~s}, 4 \mathrm{H}, \mathrm{Mes}-\mathrm{CH}), 6.44$ $\left(\mathrm{d}, \mathrm{J}=3.8 \mathrm{~Hz}, 1 \mathrm{H}\right.$, pyrrole- $H$ ). $6.11(\mathrm{~d}, J=3.8 \mathrm{~Hz}, 1 \mathrm{H}$, pyrrole- $\mathrm{H}), 2.62\left(\mathrm{~s}, 6 \mathrm{H}, \mathrm{Mes}-\mathrm{CH}_{3}\right), 2.29\left(\mathrm{~s}, 6 \mathrm{H}, \mathrm{Mes}-\mathrm{CH}_{3}\right), 2.22(\mathrm{~s}$, $3 \mathrm{H}$, Mes- $\left.\mathrm{CH}_{3}\right), 2.21\left(\mathrm{~s}, 12 \mathrm{H}\right.$, Mes- $\left.\mathrm{CH}_{3}\right), 2.12\left(\mathrm{~s}, 6 \mathrm{H}, \mathrm{Mes}-\mathrm{CH}_{3}\right), 2.04\left(\mathrm{~s}, 6 \mathrm{H}, \mathrm{Mes}-\mathrm{CH}_{3}\right), 2.03$ (s, 3H, Mes- $\left.\mathrm{CH}_{3}\right), 1.96(\mathrm{~s}$, $3 \mathrm{H}$, Mes- $\left.\mathrm{CH}_{3}\right) ;{ }^{13} \mathrm{C}\left\{{ }^{1} \mathrm{H}\right\} \mathrm{NMR}\left(\mathrm{C}_{6} \mathrm{D}_{6}, 303 \mathrm{~K}, 125 \mathrm{MHz}\right): \delta(\mathrm{ppm})=170.4$ (Aryl-C), 164.5 (Aryl-C), 155.2 (Aryl-C), 148.8 (Aryl-C), 143.6 (Aryl-C), 141.0 (Aryl-C), 137.8 (Aryl-C), 137.6 (Aryl-C), 137.6 (Aryl-C), 139.5 (Aryl-C), 137.3 (Aryl-C), 137.2 (Aryl-C), 136.8 (Aryl-C), 135.8 (Aryl-C), 135.3 (Aryl-C), 135.3 (Aryl-C), 134.0 (Aryl-C), 133.8 (pyrrole-CH), 131.8 (pyrrole- $\mathrm{CH}$ ), 128.6 (Mes-CH), 128.6 (Mes- $\mathrm{CH}$ ), 124.5 (pyrrole- $\mathrm{CH}$ ), 119.3 (pyrrole- $\mathrm{CH}$ ), 22.4 (Mes- $\mathrm{CH}_{3}$ ), 21.4 (Mes- 
$\left.\mathrm{CH}_{3}\right), 21.2\left(\mathrm{Mes}-\mathrm{CH}_{3}\right), 21.1$ (Mes- $\left.\mathrm{CH}_{3}\right), 21.0\left(\mathrm{Mes}-\mathrm{CH}_{3}\right), 20.1\left(\mathrm{Mes}-\mathrm{CH}_{3}\right), 19.6\left(\mathrm{Mes}-\mathrm{CH}_{3}\right)$. Please note that some of the Mes- $\mathrm{CH}$ Please note that some of Mes- $\mathrm{CH}^{13} \mathrm{C}$ resonances are hidden underneath the residual solvent peak of $\mathrm{C}_{6} \mathrm{D}_{6}$. ${ }^{15} \mathrm{~N} \mathrm{NMR}\left(\mathrm{C}_{6} \mathrm{D}_{6}, 303 \mathrm{~K}, 176 \mathrm{MHz}\right): \delta(\mathrm{ppm})=241.0($ pyrrole- $N), 213.7$ (pyrrole- $N$ ); elemental analysis (\%) calcd for $\mathrm{C}_{34} \mathrm{H}_{51} \mathrm{~N}_{4} \mathrm{ClMo:} \cdot \mathrm{C}_{5} \mathrm{H}_{12} \mathrm{C} 73.23$ H 7.40 N 5.79, found C 73.55 H 7.48 N 5.68.

\section{$\left(\mathrm{DPM}^{\mathrm{tBu}}\right) \mathrm{Mo}\left(\mathrm{N}^{t} \mathrm{Bu}\right)_{2} \mathrm{Cl}(3)$}

Solid KL ${ }^{2}$ (1 eq., $0.5 \mathrm{mmol}, 206 \mathrm{mg}$ ) and solid (DME) Mo( $\mathrm{N}^{t} \mathrm{Bu}_{2} \mathrm{Cl}_{2}$ (1 eq., $0.5 \mathrm{mmol}, 200 \mathrm{mg}$ ) were mixed and cooled to $-40{ }^{\circ} \mathrm{C}$. To this mixture $-40^{\circ} \mathrm{C}$ cold diethyl ether $(10 \mathrm{~mL})$ was added and the reaction mixture stirred overnight, while warming up to room temperature. The diethyl ether was evaporated from the deep orange colored reaction mixture, the reddish residue dissolved in $n$-pentane and filtered through a medium porosity frit. The solution was concentrated to $2 \mathrm{~mL}$ and left standing overnight, which resulted in the formation of orange-red needles of X-ray quality in a yield of $75 \%(0.375 \mathrm{mmol}, 243 \mathrm{mg}) .{ }^{1} \mathrm{H} \mathrm{NMR}\left(\mathrm{C}_{6} \mathrm{D}_{6}, 303 \mathrm{~K}, 700 \mathrm{MHz}\right): \delta(\mathrm{ppm})=6.83(\mathrm{~s}, 1 \mathrm{H}, \mathrm{Mes}-\mathrm{CH}), 6.71$ $(\mathrm{s}, 1 \mathrm{H}$, Mes-CH), $6.66(\mathrm{~d}, J=4.3 \mathrm{~Hz}, 1 \mathrm{H}$, pyrrole- $H), 6.64(\mathrm{~d}, J=4.3 \mathrm{~Hz}, 1 \mathrm{H}$, pyrrole- $H), 6.50(\mathrm{~d}, J=4.3 \mathrm{~Hz}, 1 \mathrm{H}$, pyrrole$H$ ), $6.24\left(\mathrm{~d}, J=4.3 \mathrm{~Hz}, 1 \mathrm{H}\right.$, pyrrole-H), $2.68\left(\mathrm{~s}, 3 \mathrm{H}, \mathrm{Mes}-\mathrm{CH}_{3}\right), 2.17\left(\mathrm{~s}, 3 \mathrm{H}, \mathrm{Mes}-\mathrm{CH}_{3}\right), 1.82\left(\mathrm{~s}, 3 \mathrm{H}, \mathrm{Mes}-\mathrm{CH}_{3}\right), 1.80(\mathrm{~s}, 9 \mathrm{H}$, pyrrole- $\left.\mathrm{C}\left(\mathrm{CH}_{3}\right)_{3}\right), 1.60\left(\mathrm{~s}, 9 \mathrm{H}, \mathrm{Mo}=\mathrm{N}-\mathrm{C}\left(\mathrm{CH}_{3}\right)_{3}\right), 1.48\left(\mathrm{~s}, 9 \mathrm{H}\right.$, pyrrole- $\left.\mathrm{C}\left(\mathrm{CH}_{3}\right)_{3}\right), 1.26\left(\mathrm{~s}, 9 \mathrm{H}, \mathrm{Mo}=\mathrm{N}-\mathrm{C}(\mathrm{CH})_{3}\right) ;{ }^{13} \mathrm{C}\left\{{ }^{1} \mathrm{H}\right\} \mathrm{NMR}$ $\left(\mathrm{C}_{6} \mathrm{D}_{6}, 303 \mathrm{~K}, 125 \mathrm{MHz}\right): \delta(\mathrm{ppm})=184.4$ (Aryl-C), 174.4 (Aryl-C), 143.3 (Aryl-C), 141.7 (Aryl-C), 138.2 (Aryl-C), 137.5 (Aryl-C), 137.4 (Aryl-C), 137.4 (Aryl-C), 134.5 (Aryl-C), 134.4 (pyrrole-CH), 131.5 (pyrrole-CH), 120.9 (pyrrole-CH), 113.7 (pyrrole- $\mathrm{CH}), 74.1\left(\mathrm{Mo}=\mathrm{N}-\mathrm{C}\left(\mathrm{CH}_{3}\right)_{3}\right), 72.8\left(\mathrm{Mo}=\mathrm{N}-\mathrm{C}\left(\mathrm{CH}_{3}\right)_{3}\right), 35.9\left(\right.$ pyrrole- $\left.\mathrm{C}\left(\mathrm{CH}_{3}\right)_{3}\right), 34.4$ (pyrrole- $\left.\mathrm{C}\left(\mathrm{CH}_{3}\right)_{3}\right), 30.8$ (pyrrole- $\left.\mathrm{C}\left(\mathrm{CH}_{3}\right)_{3}\right), 30.8$ (pyrrole- $\left.\mathrm{C}\left(\mathrm{CH}_{3}\right)_{3}\right), 30.7\left(\mathrm{Mo}=\mathrm{N}-\mathrm{C}\left(\mathrm{CH}_{3}\right)_{3}\right), 30.2\left(\mathrm{Mo}=\mathrm{N}-\mathrm{C}\left(\mathrm{CH}_{3}\right)_{3}\right), 22.1\left(\mathrm{Mes}-\mathrm{CH}_{3}\right), 21.1(\mathrm{Mes}-$ $\left.\mathrm{CH}_{3}\right), 20.0$ (Mes- $\left.\mathrm{CH}_{3}\right)$. Please note that some of the Mes- $\mathrm{CH}{ }^{13} \mathrm{C}$ resonances are hidden underneath the residual solvent peak of $\mathrm{C}_{6} \mathrm{D}_{6} .{ }^{15} \mathrm{~N}$ NMR $\left(\mathrm{C}_{6} \mathrm{D}_{6}, 303 \mathrm{~K}, 176 \mathrm{MHz}\right): \delta(\mathrm{ppm})=241.7$ (pyrrole- $\left.N\right)$, 204.8 (pyrrole- $\left.N\right), 114.8(\mathrm{Mo}=\mathrm{N}-$ $\left.\left.\left.\mathrm{CH}_{3}\right)_{3}\right), 103.9\left(\mathrm{Mo}=\mathrm{N}-\mathrm{CH}_{3}\right)_{3}\right)$; elemental analysis (\%) calcd for $\mathrm{C}_{34} \mathrm{H}_{51} \mathrm{~N}_{4} \mathrm{ClMo}$ : $\mathrm{C} 63.10 \mathrm{H}$ 7.94 N 8.66, found C 63.45 H 7.82 N 8.48 .

\section{(DPM $\left.{ }^{\mathrm{BB}}\right) \mathrm{Mo}(\mathrm{NMes})_{2} \mathrm{Cl}(4)$}

Solid KL2 (1 eq., $0.5 \mathrm{mmol}, 206 \mathrm{mg}$ ) and solid (DME)Mo(NMes) ${ }_{2} \mathrm{Cl}_{2}$ (1 eq., $0.5 \mathrm{mmol}, 260 \mathrm{mg}$ ) were mixed and cooled to $-40{ }^{\circ} \mathrm{C}$. To this mixture $-40^{\circ} \mathrm{C}$ cold diethyl ether $(10 \mathrm{~mL})$ was added and the reaction mixture stirred overnight, while warming up to room temperature and turning deep purple in color. The diethyl ether was evaporated, the reddish residue dissolved in $n$-pentane and filtered through a medium porosity frit. The solution was concentrated to $2 \mathrm{~mL}$ and left standing overnight, which resulted in the formation of purple blocks of X-ray quality in a yield of $65 \%(0.325 \mathrm{mmol}, 251 \mathrm{mg}) .{ }^{1} \mathrm{H} \mathrm{NMR}\left(\mathrm{C}_{6} \mathrm{D}_{6}, 303 \mathrm{~K}, 700 \mathrm{MHz}\right): \delta(\mathrm{ppm})=6.82(\mathrm{~d}, J=4.3 \mathrm{~Hz}, 1 \mathrm{H}$, pyrrole- $H), 6.71(\mathrm{~s}, 1 \mathrm{H}$, Mes-CH), $6.70(\mathrm{~s}, 1 \mathrm{H}$, Mes- $\mathrm{CH}$ ), $6.64(\mathrm{~s}, 2 \mathrm{H}$, Mes- $\mathrm{CH}$ ), 6.59 (d, $J=4.3 \mathrm{~Hz}, 1 \mathrm{H}$, pyrrole- $H$ ), 6.57 (s, 2H, Mes-CH), 6.49 (d, $J=4.3 \mathrm{~Hz}, 1 \mathrm{H}$, pyrrole- $H$ ), 6.32 (d, J=4.3 Hz, 1H, pyrrole- $H$ ), 2.71 (s. 6H, Mes- $\mathrm{CH}_{3}$ ), 2.27 (s. 6H, Mes- $\left.\mathrm{CH}_{3}\right), 2.14$ (s. 3H, Mes- $\mathrm{CH}_{3}$ ), 2.12 (s. 3H, Mes- $\mathrm{CH}_{3}$ ), 2.06 (s. 3H, Mes- $\mathrm{CH}_{3}$ ), 2.03 (s. 3H, Mes- $\mathrm{CH}_{3}$ ), 1.87 (s. 3H, Mes- $\mathrm{CH}_{3}$ ), 1.77 (pyrrole- $\left.\mathrm{C}\left(\mathrm{CH}_{3}\right)_{3}\right), 1.42$ (pyrrole- $\left.\mathrm{C}\left(\mathrm{CH}_{3}\right)_{3}\right) ;{ }^{13} \mathrm{C}\left\{{ }^{1} \mathrm{H}\right\} \mathrm{NMR}\left(\mathrm{C}_{6} \mathrm{D}_{6}, 303 \mathrm{~K}, 125 \mathrm{MHz}\right): \delta(\mathrm{ppm})=184.1$ (Aryl-C), 173.3 (ArylC), 155.1 (Aryl-C), 155.0 (Aryl-C), 144.4 (Aryl-C), 143.1 (Aryl-C), 142.2 (Aryl-C), 140.0 (Aryl-C), 137.9 (Aryl-C), 137.5 (Aryl-C), 137.1 (Aryl-C), 135.9 (Aryl-C), 135.2 (Aryl-C), 134.9 (Aryl-C), 134.8 (pyrrole-CH), 131.0 (pyrrole-CH), 129.6 (Mes- $\mathrm{CH}$ ), 128.6 (Mes- $\mathrm{CH}$ ), 120.1 (pyrrole- $\mathrm{CH}), 115.2$ (pyrrole- $\mathrm{CH}), 36.1$ (pyrrole- $\left.\mathrm{C}\left(\mathrm{CH}_{3}\right)_{3}\right), 34.7$ (pyrrole- $\left.\mathrm{C}\left(\mathrm{CH}_{3}\right)_{3}\right)$, 31.4 (pyrrole- $\left.\mathrm{C}\left(\mathrm{CH}_{3}\right)_{3}\right), 31.2$ (pyrrole- $\left.\mathrm{C}\left(\mathrm{CH}_{3}\right)_{3}\right), 21.1\left(\mathrm{Mes}-\mathrm{CH}_{3}\right), 21.0\left(\mathrm{Mes}-\mathrm{CH}_{3}\right), 20.9\left(\mathrm{Mes}-\mathrm{CH}_{3}\right), 20.4\left(\mathrm{Mes}-\mathrm{CH}_{3}\right), 20.0$ $\left(\right.$ Mes- $\left.\mathrm{CH}_{3}\right), 19.9\left(\mathrm{Mes}-\mathrm{CH}_{3}\right) ;{ }^{15} \mathrm{~N} \mathrm{NMR}\left(\mathrm{C}_{6} \mathrm{D}_{6}, 303 \mathrm{~K}, 176 \mathrm{MHz}\right): \delta(\mathrm{ppm})=241.7$ (pyrrole- $\left.N\right), 210.5$ (pyrrole- $N$ ); elemental analysis (\%) calcd for $\mathrm{C}_{44} \mathrm{H}_{55} \mathrm{~N}_{4} \mathrm{ClMo}$ : $\mathrm{C} 68.51 \mathrm{H} 7.19 \mathrm{~N} 7.26$, found C $68.32 \mathrm{H} 7.24 \mathrm{~N} 7.34$.

\section{$\left(\mathrm{DPM}^{\mathrm{Mes}}\right) \mathrm{Mo}\left(\mathrm{N}^{t} \mathrm{Bu}\right)_{2}$ (5)}

Complex 1 ( 1 eq, $0.2 \mathrm{mmol}, 154 \mathrm{mg}$ ) was dissolved in THF ( $5 \mathrm{~mL}$ ) and stirred for $5 \mathrm{~min}$ at room temperature. To this solution was added solid $\mathrm{KC}_{8}(1.5$ eq., $0.3 \mathrm{mmol}, 41 \mathrm{mg})$ and the reaction mixture was stirred for $5 \mathrm{~h}$ at room temperature during which the pink-red color of 1 changed to dark orange-pink of $\mathbf{5}$. The solvent was evaporated under high vacuum and the residue redissolved in benzene and filtered to remove graphite and unreacted potassium graphite. The benzene filtrate was frozen and sublimed under vacuum to give pure $\mathbf{5}$ as an orange-pink powder in a yield of $74 \%(0.148 \mathrm{mmol}, 109 \mathrm{mg})$. X-ray quality crystals were obtained by slow evaporation of a diethyl ether solution of 5 at room temperature over $2 \mathrm{~d}$. The NMR spectra of $\mathbf{5}$ only contained only very broad features which prevented any useful assignments and integrations. Evans method $\left(\mathrm{C}_{6} \mathrm{D}_{6}, 303 \mathrm{~K}\right): \mu_{\mathrm{eff}}=1.56 \mu_{\mathrm{B}}$; elemental analysis (\%) calcd for $\mathrm{C}_{44} \mathrm{H}_{55} \mathrm{~N}_{4} \mathrm{Mo}$ : C 71.81 H $7.53 \mathrm{~N} 7.61$, found C 71.97 H 7.75 N 7.27.

\section{$\left(\mathrm{DPM}^{\mathrm{Mes}}\right) \mathrm{Mo}\left(\mathrm{N}^{t} \mathrm{Bu}\right)_{2}$ (OTf) (6)}

Complex 1 (1 eq., $0.1 \mathrm{mmol}, 77 \mathrm{mg}$ ) was dissolved in THF $(5 \mathrm{~mL}$ ) and stirred for $5 \mathrm{~min}$ at room temperature. To this solution was added solid silver triflate (1.05 eq., $0.105 \mathrm{mmol}, 27 \mathrm{mg}$ ), causing a color change to a slightly lighter pink tone. The reaction mixture was stirred overnight at room temperature and the solvent were then evaporated under high vacuum. The pink-red residue was redissolved in $n$-pentane $(10 \mathrm{~mL})$ and filtered through glass fibre filter. The $n$-pentane solution was concentrated to $2 \mathrm{~mL}$ and stored at room temperature overnight, resulting in the deposition of large X-ray quality blocks of desired complex 6 in a yield of $69 \%(0.069 \mathrm{mmol}, 61 \mathrm{mg}) .{ }^{1} \mathrm{H} \mathrm{NMR}\left(\mathrm{C}_{6} \mathrm{D}_{6}, 303 \mathrm{~K}\right.$, $700 \mathrm{MHz}): \delta(\mathrm{ppm})=7.00(\mathrm{~s}, 1 \mathrm{H}$, Mes- $\mathrm{CH}), 6.86(\mathrm{~s}, 1 \mathrm{H}$, Mes- $\mathrm{CH}), 6.83(\mathrm{~s}, 1 \mathrm{H}$, Mes-CH), $6.79(\mathrm{~s}, 1 \mathrm{H}, \mathrm{Mes}-\mathrm{CH}), 6.74(\mathrm{~s}$, 
$1 \mathrm{H}$, Mes- $\mathrm{CH}$ ), $6.74(\mathrm{~s}, 1 \mathrm{H}$, Mes-CH), $6.63(\mathrm{~d}, J=4.1 \mathrm{~Hz}, 1 \mathrm{H}$, pyrrole- $H), 6.61(\mathrm{~d}, J=4.1 \mathrm{~Hz}, 1 \mathrm{H}$, pyrrole- $H), 6.38(\mathrm{~d}, \mathrm{~J}=$ $4.1 \mathrm{~Hz}, 1 \mathrm{H}$, pyrrole- $H$ ), $5.94\left(\mathrm{~d}, J=4.1 \mathrm{~Hz}, 1 \mathrm{H}\right.$, pyrrole- $H$ ), $2.66\left(\mathrm{~s}, 3 \mathrm{H}, \mathrm{Mes}-\mathrm{CH}_{3}\right), 2.58\left(\mathrm{~s}, 3 \mathrm{H}, \mathrm{Mes}-\mathrm{CH}_{3}\right), 2.52(\mathrm{~s}, 3 \mathrm{H}$, Mes- $\left.\mathrm{CH}_{3}\right), 2.39\left(\mathrm{~s}, 3 \mathrm{H}, \mathrm{Mes}-\mathrm{CH}_{3}\right), 2.23\left(\mathrm{~s}, 3 \mathrm{H}, \mathrm{Mes}-\mathrm{CH}_{3}\right), 2.16\left(\mathrm{~s}, 3 \mathrm{H}, \mathrm{Mes}-\mathrm{CH}_{3}\right), 2.12\left(\mathrm{~s}, 3 \mathrm{H}, \mathrm{Mes}-\mathrm{CH}_{3}\right), 2.03(\mathrm{~s}, 3 \mathrm{H}$, Mes- $\left.\mathrm{CH}_{3}\right), 1.99\left(\mathrm{~s}, 3 \mathrm{H}\right.$, Mes- $\left.\mathrm{CH}_{3}\right), 1.43\left(\mathrm{~s}, 9 \mathrm{H}, \mathrm{Mo}=\mathrm{N}-\mathrm{C}\left(\mathrm{CH}_{3}\right)_{3}\right), 0.92\left(\mathrm{~s}, 9 \mathrm{H}, \mathrm{Mo}=\mathrm{N}-\mathrm{C}\left(\mathrm{CH}_{3}\right)_{3}\right) ;{ }^{13} \mathrm{C}\left\{{ }^{1} \mathrm{H}\right\} \mathrm{NMR}\left(\mathrm{C}_{6} \mathrm{D}_{6}, 303 \mathrm{~K}\right.$, $125 \mathrm{MHz}): \delta(\mathrm{ppm})=19.3\left(\mathrm{Mes}-\mathrm{CH}_{3}\right), 20.5\left(\mathrm{Mes}-\mathrm{CH}_{3}\right), 20.9$, $\left(\mathrm{Mes}-\mathrm{CH}_{3}\right), 21.0\left(\mathrm{Mes}-\mathrm{CH}_{3}\right), 21.2\left(\mathrm{Mes}-\mathrm{CH}_{3}\right), 22.2(\mathrm{Mes}-$ $\left.\mathrm{CH}_{3}\right), 22.3\left(\mathrm{Mes}-\mathrm{CH}_{3}\right), 22.9\left(\mathrm{Mes}-\mathrm{CH}_{3}\right), 23.6\left(\mathrm{Mes}-\mathrm{CH}_{3}\right), 29.8\left(\mathrm{Mo}=\mathrm{N}-\mathrm{C}\left(\mathrm{CH}_{3}\right)_{3}\right) .31 .7\left(\mathrm{Mo}=\mathrm{N}-\mathrm{C}\left(\mathrm{CH}_{3}\right)_{3}\right), 74.9(\mathrm{Mo}=\mathrm{N}-$ $\left.\mathrm{C}\left(\mathrm{CH}_{3}\right)_{3}\right), 76.3\left(\mathrm{Mo}=\mathrm{N}-\mathrm{C}\left(\mathrm{CH}_{3}\right)_{3}\right), 120.2$ (pyrrole- $\left.\mathrm{CH}\right), 123.5$ (pyrrole- $\left.\mathrm{CH}\right), 128.7$ (Mes- $\left.\mathrm{CH}\right), 128.8$ (Mes- $\left.\mathrm{CH}\right), 128.9$ (Mes$\mathrm{CH}$ ), 130.0 (Mes- $\mathrm{CH}$ ), 132.0 (pyrrole- $\mathrm{CH}$ ), 132.2 (pyrrole- $\mathrm{CH}$ ), 133.1 (Aryl-C), 133.9 (Aryl-C), 134.0 (Aryl-C), 136.4 (ArylC), 136.8 (Aryl-C), 137.0 (Aryl-C), 138.0 (Aryl-C), 135.5 (Aryl-C), 138.7 (Aryl-C), 139.3 (Aryl-C), 140.4 (Aryl-C), 142.6 (Aryl-C), 145.8 (Aryl-C), 165.6 (Aryl-C), $166.8\left(\right.$ Aryl-C) ${ }^{15} \mathrm{~N} \mathrm{NMR}\left(\mathrm{C}_{6} \mathrm{D}_{6}, 303 \mathrm{~K}, 176 \mathrm{MHz}\right): \delta(\mathrm{ppm})=242.4$ (pyrrole- $\left.N\right)$, 208.4 (pyrrole- $N)$, $124.4\left(\mathrm{Mo}=\mathrm{N}-\mathrm{C}\left(\mathrm{CH}_{3}\right)_{3}\right), 122.3\left(\mathrm{Mo}=\mathrm{N}-\mathrm{C}\left(\mathrm{CH}_{3}\right)_{3}\right) ;{ }^{19} \mathrm{~F} \mathrm{NMR}\left(\mathrm{C}_{6} \mathrm{D}_{6}, 303 \mathrm{~K}, 659 \mathrm{MHz}\right): \delta(\mathrm{ppm})=-72.36$ (OTf); elemental analysis (\%) calcd for $\mathrm{C}_{45} \mathrm{H}_{55} \mathrm{~N}_{4} \mathrm{O}_{3} \mathrm{~F}_{3} \mathrm{SMo}$ : $\mathrm{C} 61.07 \mathrm{H} 6.26 \mathrm{~N} \mathrm{6.33}$, found $\mathrm{C} 60.68 \mathrm{H} 5.88 \mathrm{~N} 6.21$.

\section{$\left(\mathrm{Me}_{3} \mathrm{SiCH}_{2}-\mathrm{DPM}^{\mathrm{Mes}}\right) \mathrm{Mo}\left(\mathrm{N}^{\mathrm{t}} \mathrm{Bu}\right)_{2}(7)$}

Complex 1 (1 eq., $0.2 \mathrm{mmol}, 154 \mathrm{mg}$ ) was dissolved in diethyl ether $(5 \mathrm{~mL}$ ) and stirred for 5 min at room temperature. To this solution was added $\mathrm{LiCH}_{2} \mathrm{SiMe}_{3}(1.3$ eq., $0.26 \mathrm{mmol}, 25 \mathrm{mg}$ ) dissolved in diethyl ether $(2 \mathrm{~mL})$, causing a fast color change from red-pink to green. The reaction mixture was stirred overnight at room temperature and the solvent was evaporated. The dark green residue was dissolved in $n$-pentane, filtered and concentrated to $1 \mathrm{~mL}$. Dark green X-ray quality crystals deposited over the course of one night in a yield of $55 \%(0.11 \mathrm{mmol}, 92 \mathrm{mg})$. ${ }^{1} \mathrm{H}$ NMR $\left(\mathrm{C}_{6} \mathrm{D}_{6}, 303 \mathrm{~K}, 700 \mathrm{MHz}\right): \delta(\mathrm{ppm})=6.98(\mathrm{~s}, 1 \mathrm{H}$, Mes- $\mathrm{CH}), 6.97(\mathrm{~s}, 1 \mathrm{H}$, Mes-CH), 6.87, (s, 1H, Mes-CH), $6.84(\mathrm{~s}$, $2 \mathrm{H}$, Mes- $\mathrm{CH}), 6.82(\mathrm{~s}, 1 \mathrm{H}$, Mes- $\mathrm{CH}), 6.56(\mathrm{~d}, J=3.3 \mathrm{~Hz}, 1 \mathrm{H}$, pyrrole- $H), 6.42(\mathrm{~d}, J=3.3 \mathrm{~Hz}, 1 \mathrm{H}$, pyrrole- $H), 5.31(\mathrm{~d}, J=$ $3.3 \mathrm{~Hz}, 1 \mathrm{H}$, pyrrole- $H$ ), $3.68\left(\mathrm{dt}, J=12.8 \mathrm{~Hz}, J=2.4 \mathrm{~Hz}, 1 \mathrm{H}\right.$, pyrrole- $\left.\mathrm{H}\left(\mathrm{CH}_{2} \mathrm{TMS}\right)\right), 2.55\left(\mathrm{~s}, 3 \mathrm{H}, \mathrm{Mes}-\mathrm{CH}_{3}\right), 2.45(\mathrm{~s}, 3 \mathrm{H}$, Mes- $\left.\mathrm{CH}_{3}\right), 2.36\left(\mathrm{~s}, 3 \mathrm{H}, \mathrm{Mes}-\mathrm{CH}_{3}\right), 2.33\left(\mathrm{~s}, 3 \mathrm{H}, \mathrm{Mes}-\mathrm{CH}_{3}\right), 2.29\left(\mathrm{~s}, 3 \mathrm{H}, \mathrm{Mes}-\mathrm{CH}_{3}\right), 2.26\left(\mathrm{~s}, 3 \mathrm{H}, \mathrm{Mes}-\mathrm{CH}_{3}\right), 2.22(\mathrm{~s}, 3 \mathrm{H}$, Mes- $\left.\mathrm{CH}_{3}\right), 2.20\left(\mathrm{~s}, 3 \mathrm{H}, \mathrm{Mes}-\mathrm{CH}_{3}\right), 2.18\left(\mathrm{~s}, 3 \mathrm{H}, \mathrm{Mes}-\mathrm{CH}_{3}\right), 1.33\left(\mathrm{~s}, 9 \mathrm{H}, \mathrm{Mo}=\mathrm{N}-\mathrm{C}\left(\mathrm{CH}_{3}\right)_{3}\right), 0.87(\mathrm{t}, J=12.8 \mathrm{~Hz}, 2 \mathrm{H}$, $\left.\mathrm{CH}_{2} \mathrm{Si}\left(\mathrm{CH}_{3}\right)_{3}\right), 0.78\left(\mathrm{~s}, 9 \mathrm{H}, \mathrm{Mo}=\mathrm{N}-\mathrm{C}\left(\mathrm{CH}_{3}\right)_{3}\right),-0.14\left(\mathrm{~s}, 9 \mathrm{H}, \mathrm{CH}_{2} \mathrm{Si}\left(\mathrm{CH}_{3}\right)_{3}\right) ;{ }^{13} \mathrm{C}\{1 \mathrm{H}\} \mathrm{NMR}\left(\mathrm{C}_{6} \mathrm{D}_{6}, 303 \mathrm{~K}, 125 \mathrm{MHz}\right): \delta(\mathrm{ppm})=$ 153.0 (Aryl-C), 149.0 (Aryl-C), 142.0 (Aryl-C), 140.6 (Aryl-C), 138.7 (Aryl-C), 138.7 (Aryl-C), 137.7 (Aryl-C), 137.5 (ArylC), 136.8 (Aryl-C), 136.8 (Aryl-C), 136.8 (Aryl-C), 136.2 (Aryl-C), 136.1 (Aryl-C), 132.0 (Aryl-C), 129.1 (Mes-CH), 128.7 (Mes- $\mathrm{CH}$ ), 128.7 (Mes- $\mathrm{CH}$ ), 128.5 (Mes- $\mathrm{CH}$ ), 121.7 (Aryl- $\mathrm{C}), 117.5$ (pyrrole- $\mathrm{CH}$ ), 112.9 (pyrrole- $\mathrm{CH}$ ), 112.2 (pyrrole$\mathrm{CH}), 70.8\left(\mathrm{Mo}=\mathrm{N}-\mathrm{C}\left(\mathrm{CH}_{3}\right)_{3}\right), 69.6\left(\mathrm{Mo}=\mathrm{N}-\mathrm{C}\left(\mathrm{CH}_{3}\right)_{3}\right), 47.1\left(\mathrm{CH}_{2} \mathrm{Si}\left(\mathrm{CH}_{3}\right)_{3}-\right.$ pyrrole- $\left.\mathrm{CH}\right), 33.1\left(\mathrm{Mo}=\mathrm{N}-\mathrm{C}\left(\mathrm{CH}_{3}\right)_{3}\right), 30.7(\mathrm{Mo}=\mathrm{N}-$ $\left.\mathrm{C}\left(\mathrm{CH}_{3}\right)_{3}\right), 21.7\left(\mathrm{Mes}-\mathrm{CH}_{3}\right), 21.2\left(\mathrm{Mes}-\mathrm{CH}_{3}\right), 21.2\left(\mathrm{CH}_{2} \mathrm{Si}\left(\mathrm{CH}_{3}\right)_{3}\right), 21.2\left(\mathrm{Mes}-\mathrm{CH}_{3}\right), 21.2\left(\mathrm{Mes}-\mathrm{CH}_{3}\right), 20.6\left(\mathrm{Mes}-\mathrm{CH}_{3}\right), 20.2$ (Mes- $\left.\mathrm{CH}_{3}\right), 20.2\left(\mathrm{Mes}-\mathrm{CH}_{3}\right), 20.2\left(\mathrm{Mes}-\mathrm{CH}_{3}\right), 19.6\left(\mathrm{Mes}-\mathrm{CH}_{3}\right),-1.2\left(\mathrm{CH}_{2} \mathrm{Si}\left(\mathrm{CH}_{3}\right)_{3}\right) ;{ }^{15} \mathrm{~N} \mathrm{NMR}\left(\mathrm{C}_{6} \mathrm{D}_{6}, 303 \mathrm{~K}, 176 \mathrm{MHz}\right): \delta$ $($ ppm $)=31.8\left(\mathrm{Mo}=\mathrm{N}-\mathrm{C}\left(\mathrm{CH}_{3}\right)_{3}\right), 100.2\left(\mathrm{Mo}=\mathrm{N}-\mathrm{C}\left(\mathrm{CH}_{3}\right)_{3}\right), 228.3($ pyrrole- $N), 250.3\left(\mathrm{CH}_{2} \mathrm{Si}\left(\mathrm{CH}_{3}\right)_{3}-\right.$ pyrrole- $\left.N\right) ;{ }^{29} \mathrm{Si}\left\{{ }^{1} \mathrm{H}\right\} \mathrm{NMR}$ $\left(\mathrm{C}_{6} \mathrm{D}_{6}, 303 \mathrm{~K}, 176 \mathrm{MHz}\right): \delta(\mathrm{ppm})=0.82\left(\mathrm{CH}_{2} \mathrm{Si}\left(\mathrm{CH}_{3}\right)_{3}\right)$; elemental analysis (\%) calcd for $\mathrm{C}_{49} \mathrm{H}_{67} \mathrm{~N}_{4} \mathrm{SiMo:} \mathrm{C} 70.39 \mathrm{H} 8.08$ N 6.70, found C 70.54 H 7.94 N 6.31 . 


\section{NMR spectra}

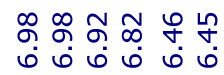

ทู่ กูก กูก

ini

पî

पर
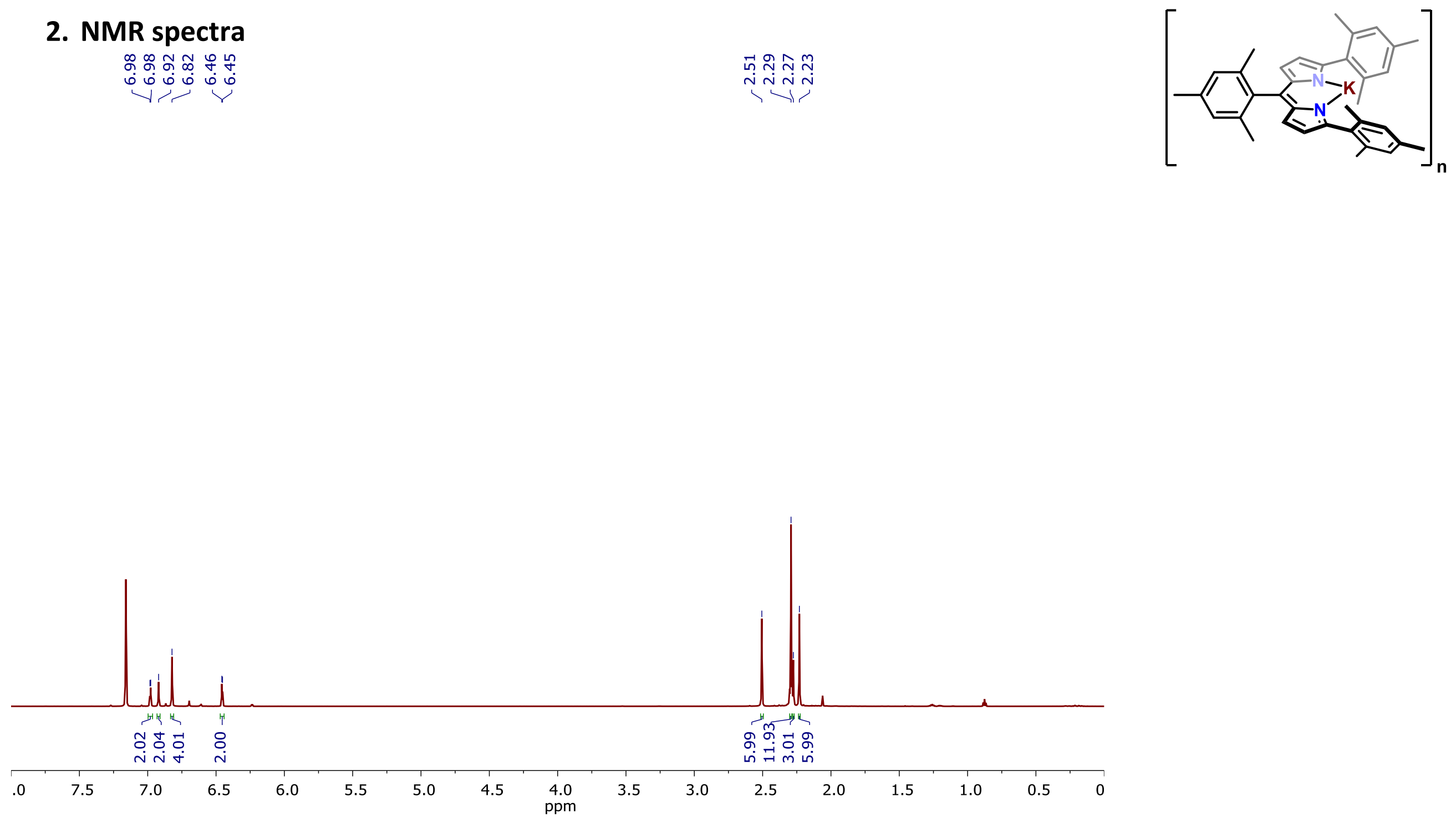

Figure S1: $\quad{ }^{1} \mathrm{H}$ NMR spectrum of $\mathrm{KL}^{1}$ in $\mathrm{C}_{6} \mathrm{D}_{6}$ at $303 \mathrm{~K}$. 

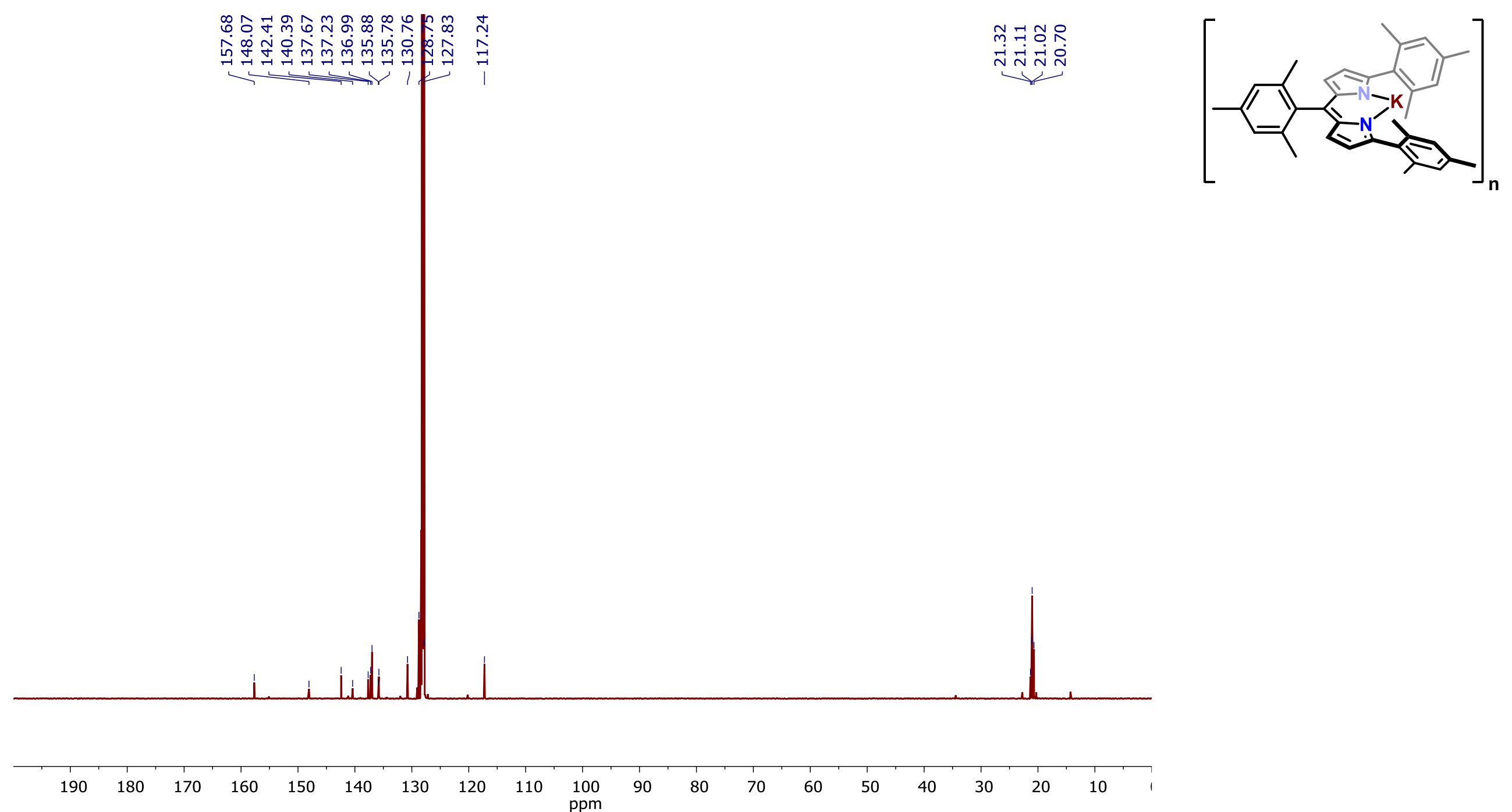

Figure S2: $\quad{ }^{13} \mathrm{C}\left\{{ }^{1} \mathrm{H}\right\}$ NMR spectrum of $\mathrm{KL}^{\mathbf{1}}$ in $\mathrm{C}_{6} \mathrm{D}_{6}$ at $303 \mathrm{~K}$. 


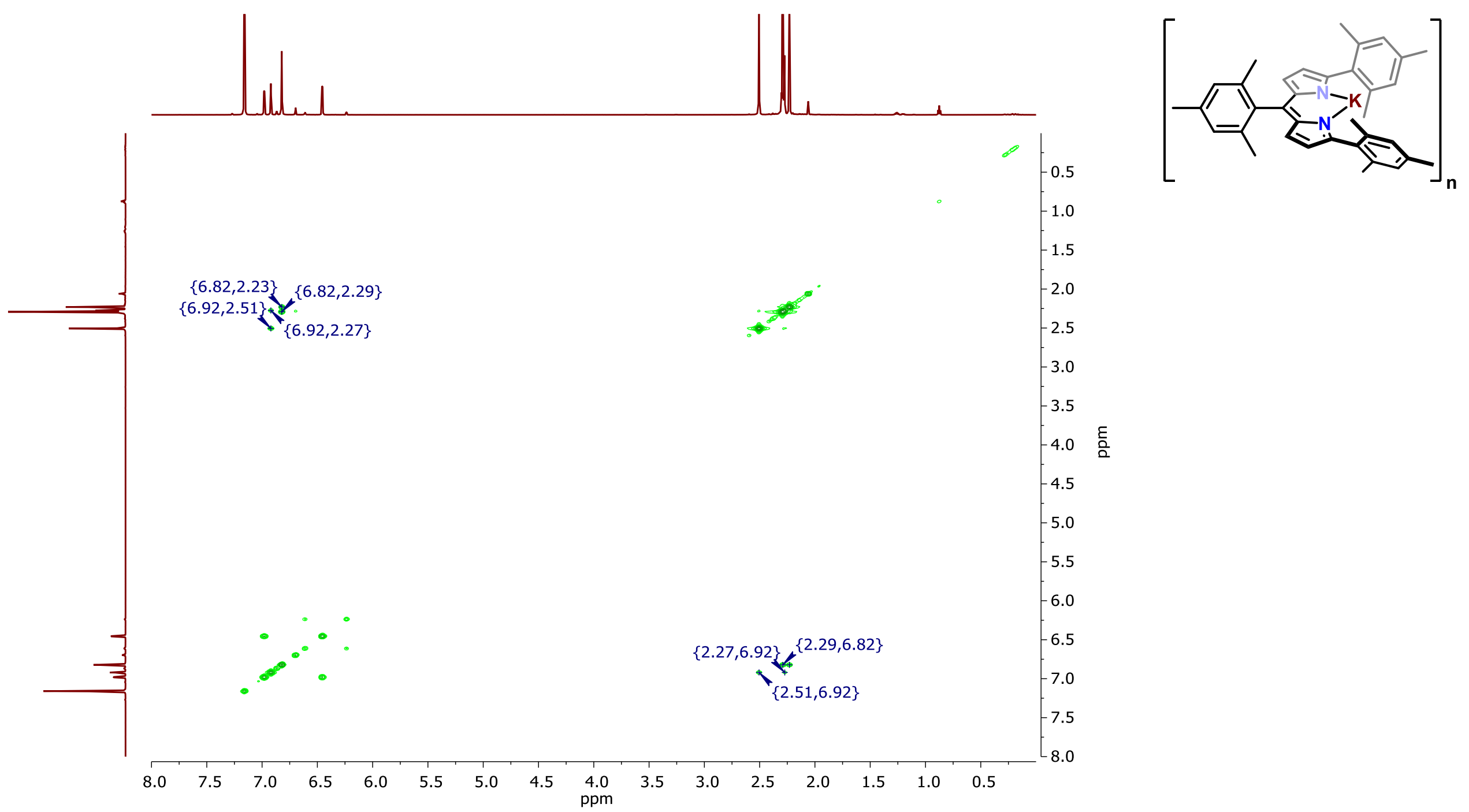

Figure S3: $\quad{ }^{1} \mathrm{H}-{ }^{1} \mathrm{H}$ COSY NMR spectrum of $\mathrm{KL}^{1}$ in $\mathrm{C}_{6} \mathrm{D}_{6}$ at $303 \mathrm{~K}$. 


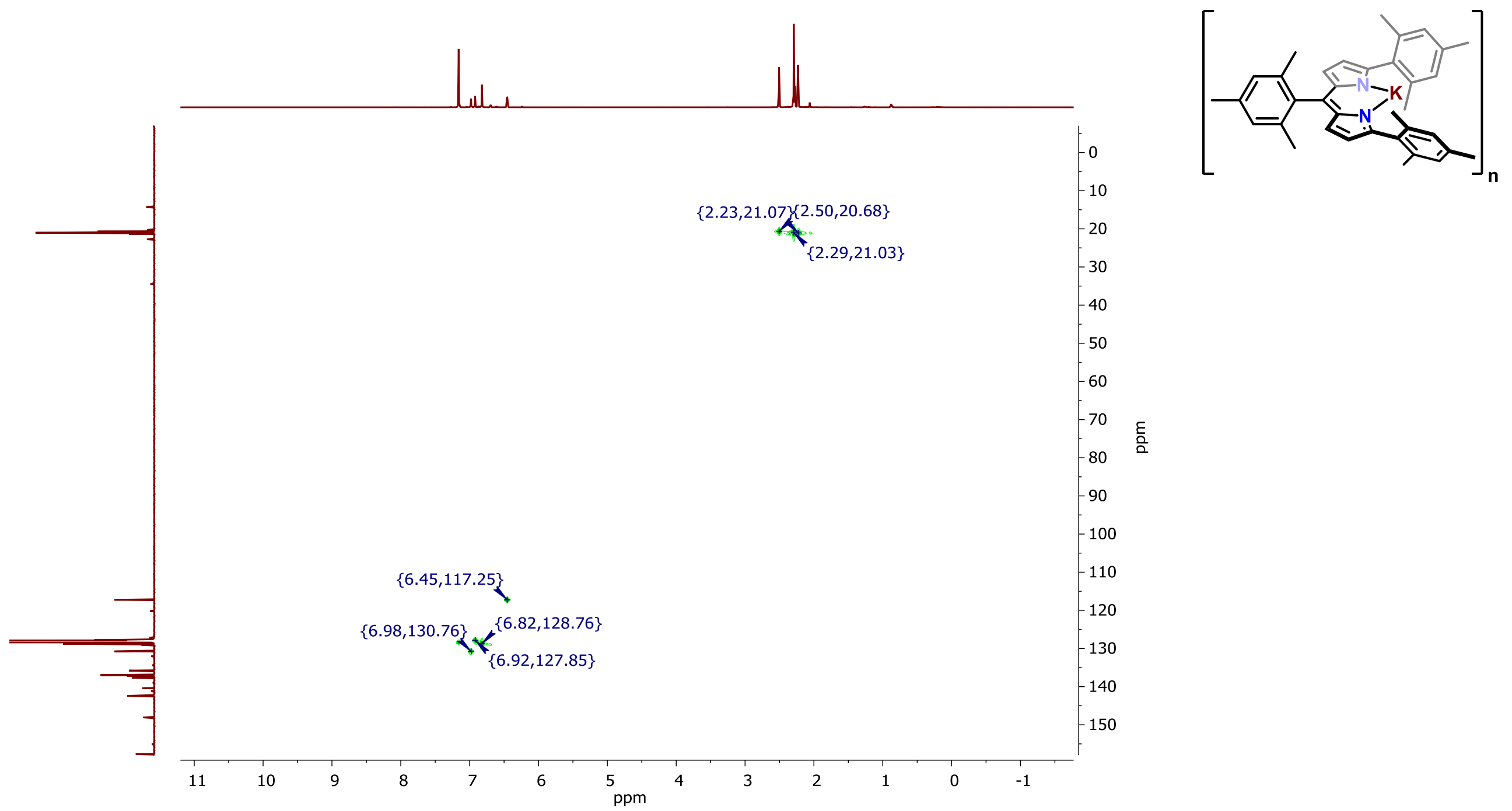

Figure S4: $\quad{ }^{1} \mathrm{H}-{ }^{13} \mathrm{C}$ HSQC NMR spectrum of $\mathrm{KL}^{1}$ in $\mathrm{C}_{6} \mathrm{D}_{6}$ at $303 \mathrm{~K}$. 


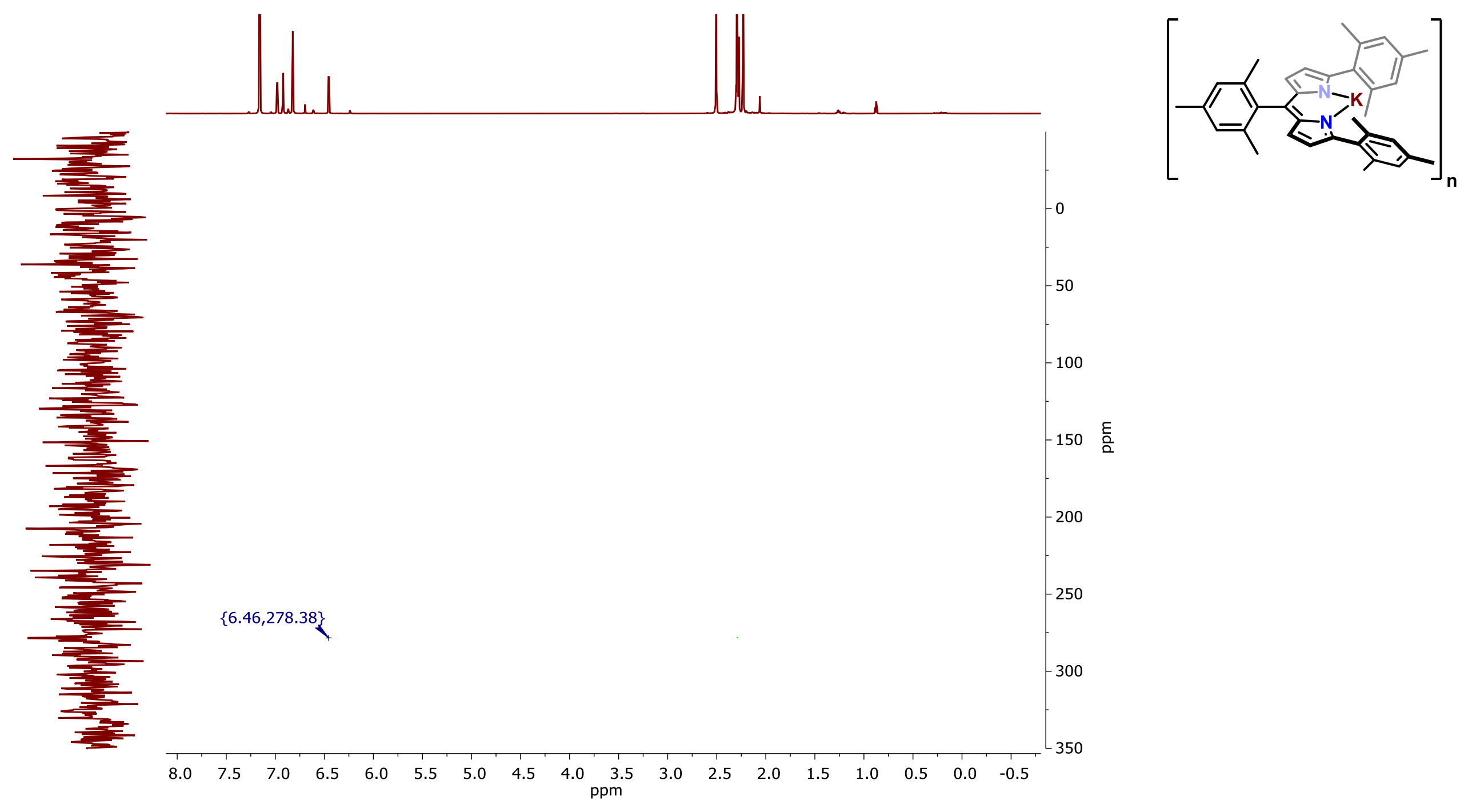

Figure S5: $\quad{ }^{1} \mathrm{H}-{ }^{15} \mathrm{~N}$ HMBC NMR spectrum of $\mathrm{KL}^{1}$ in $\mathrm{C}_{6} \mathrm{D}_{6}$ at $303 \mathrm{~K}$. 


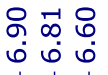

$$
\begin{aligned}
& \begin{array}{ll}
0 & 0 \\
1 & 0
\end{array}
\end{aligned}
$$

$\stackrel{\sim}{\sim} \stackrel{\text { iे }}{\sim}$

$\stackrel{\infty}{\stackrel{\infty}{+}}$

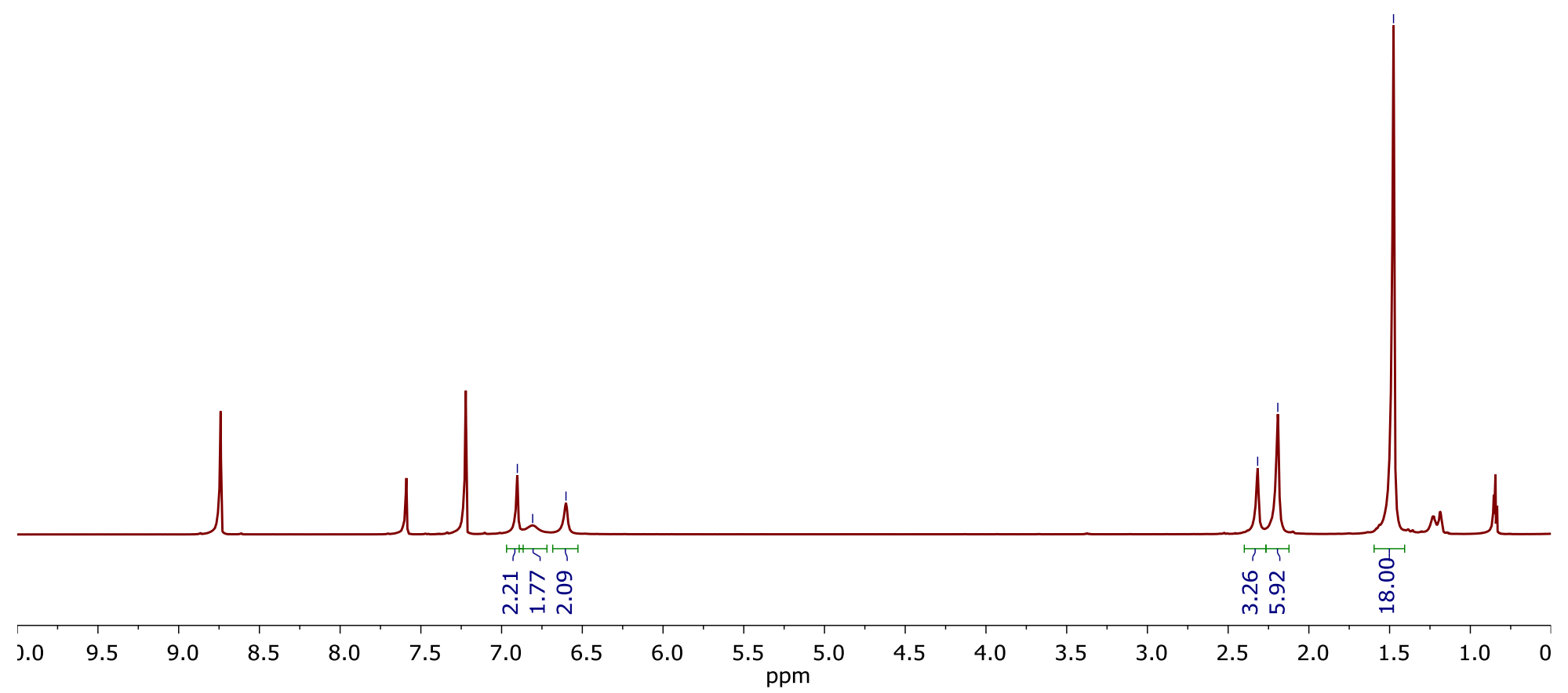

Figure S6: $\quad{ }^{1} \mathrm{H}$ NMR spectrum of $\mathrm{KL}^{2}$ in py- $\mathrm{d}_{5}$ at $303 \mathrm{~K}$.

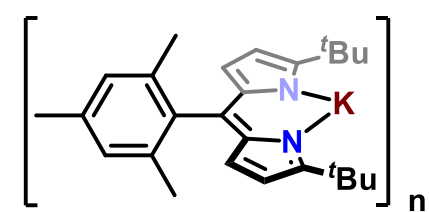



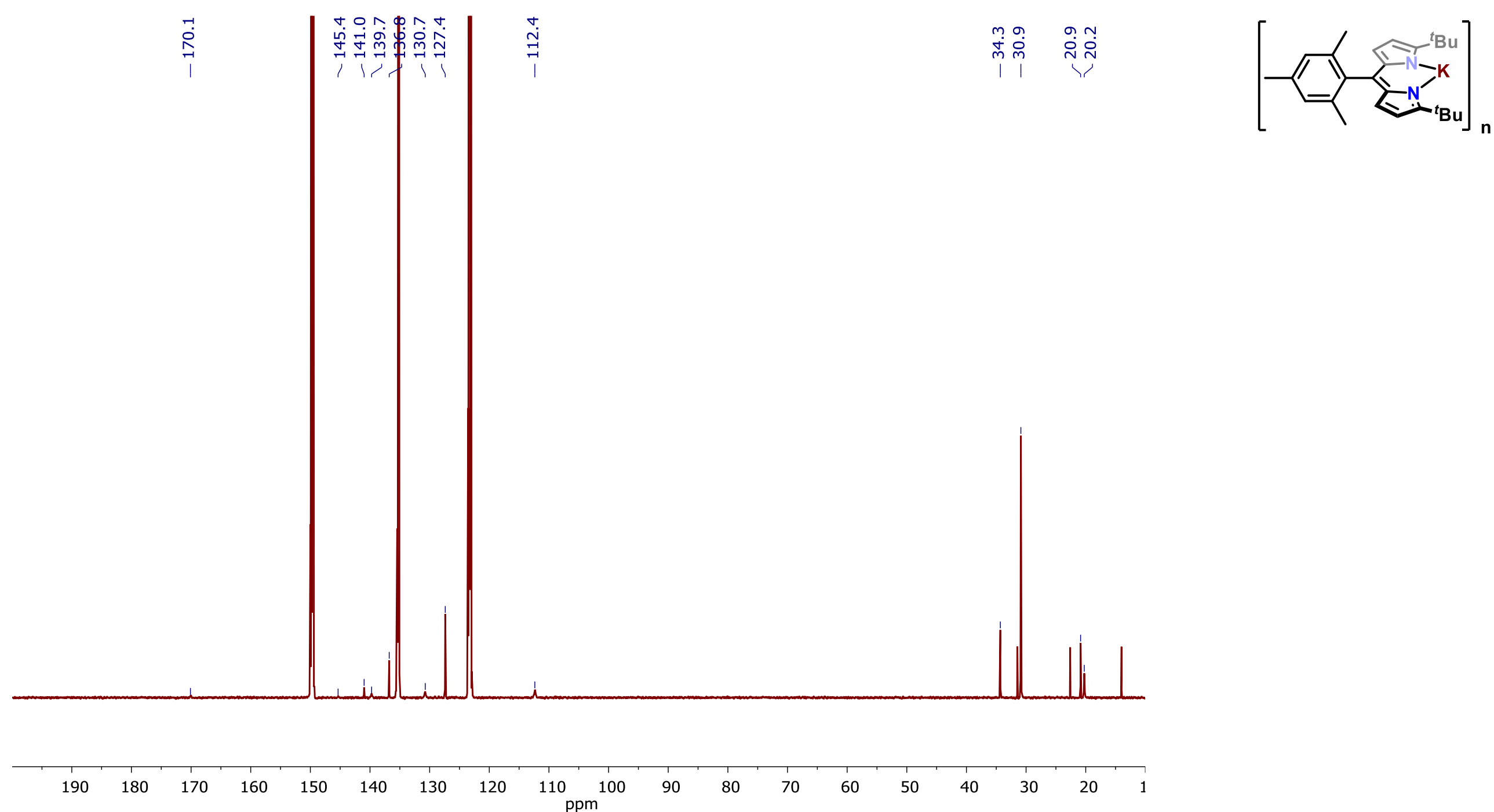

Figure S7: $\quad{ }^{13} \mathrm{C}\left\{{ }^{1} \mathrm{H}\right\}$ NMR spectrum of $\mathrm{KL}^{2}$ in py-d at $303 \mathrm{~K}$. 

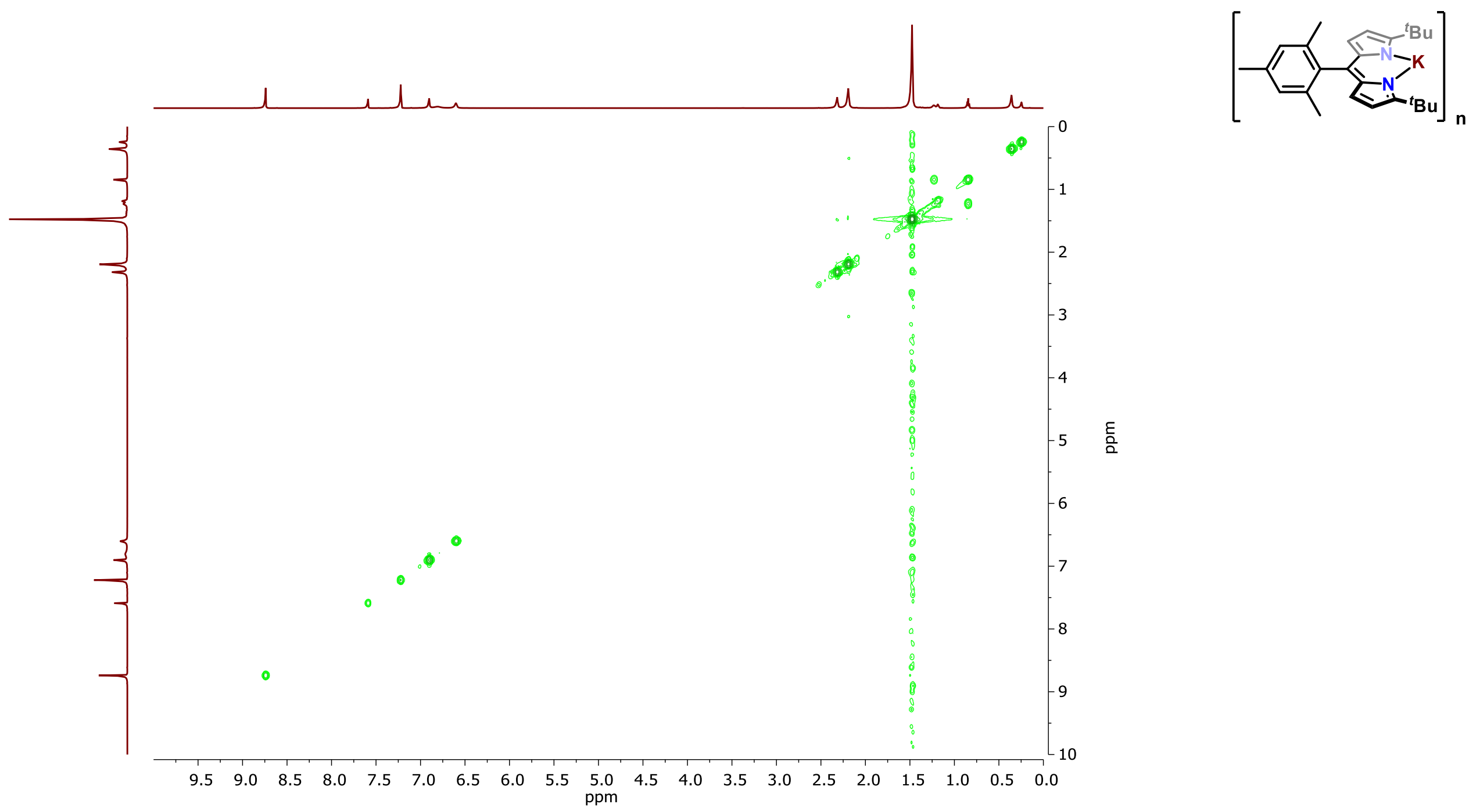

Figure S8: $\quad{ }^{1} \mathrm{H}-{ }^{1} \mathrm{H}$ COSY NMR spectrum of $\mathrm{KL}^{2}$ in py- $\mathrm{d}_{5}$ at $303 \mathrm{~K}$. 

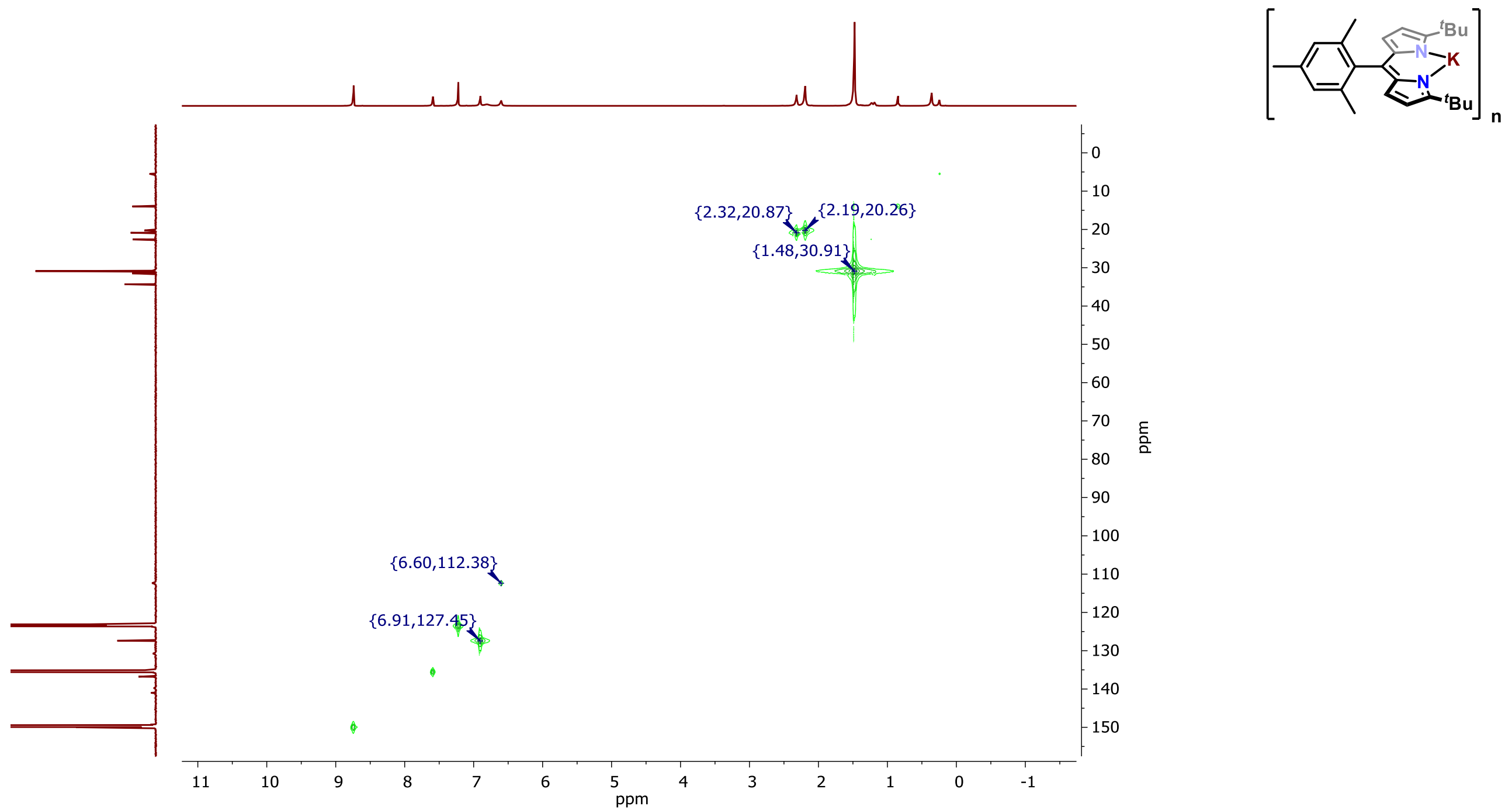

Figure S9: $\quad{ }^{1} \mathrm{H}-{ }^{13} \mathrm{C} \mathrm{HSQC} \mathrm{NMR} \mathrm{spectrum} \mathrm{of} \mathrm{KL}^{2}$ in py-d $\mathrm{d}_{5}$ at $303 \mathrm{~K}$. 

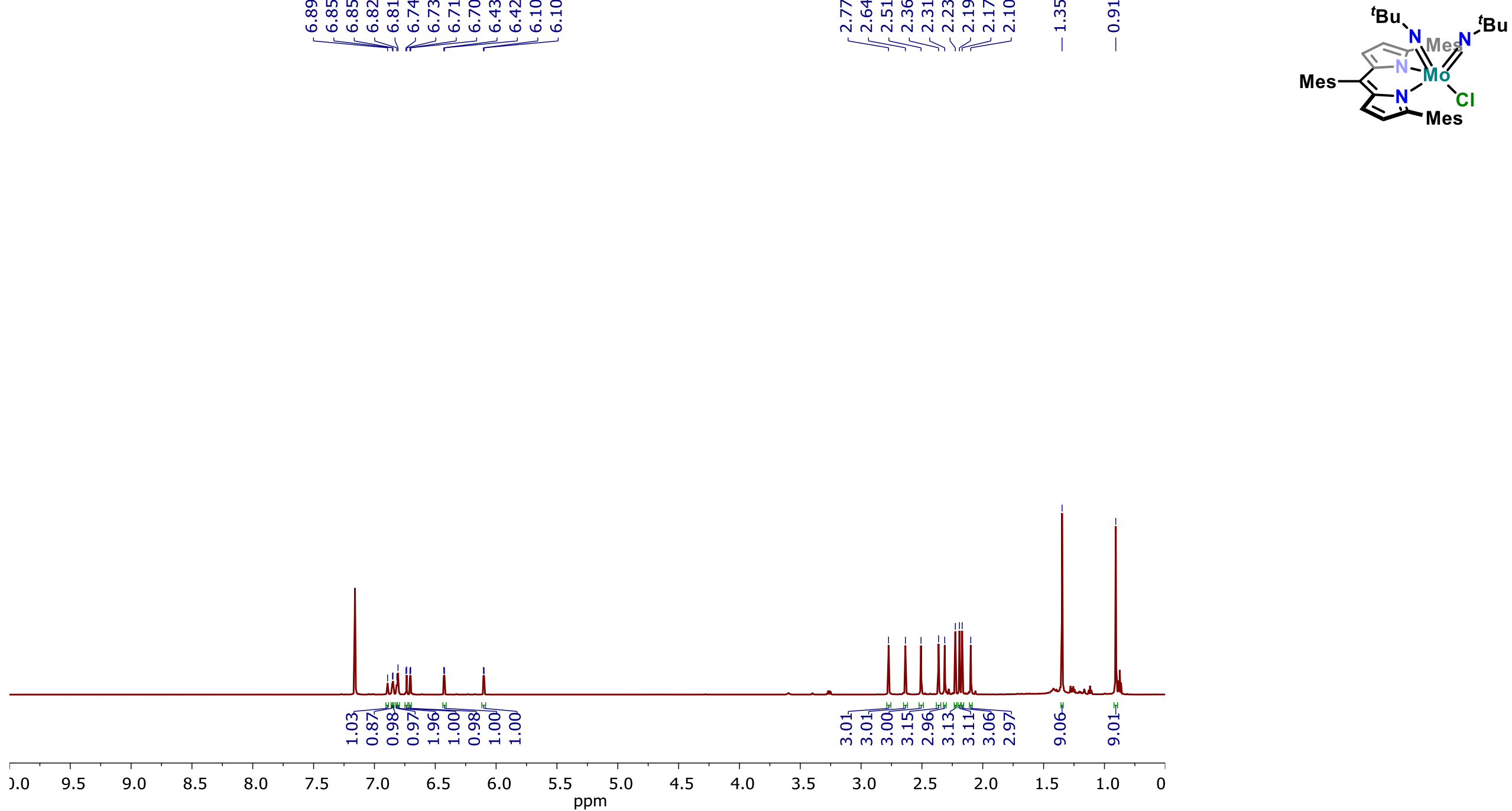

Figure S10: $\quad{ }^{1} \mathrm{H}$ NMR spectrum of 1 in $\mathrm{C}_{6} \mathrm{D}_{6}$ at $303 \mathrm{~K}$ 

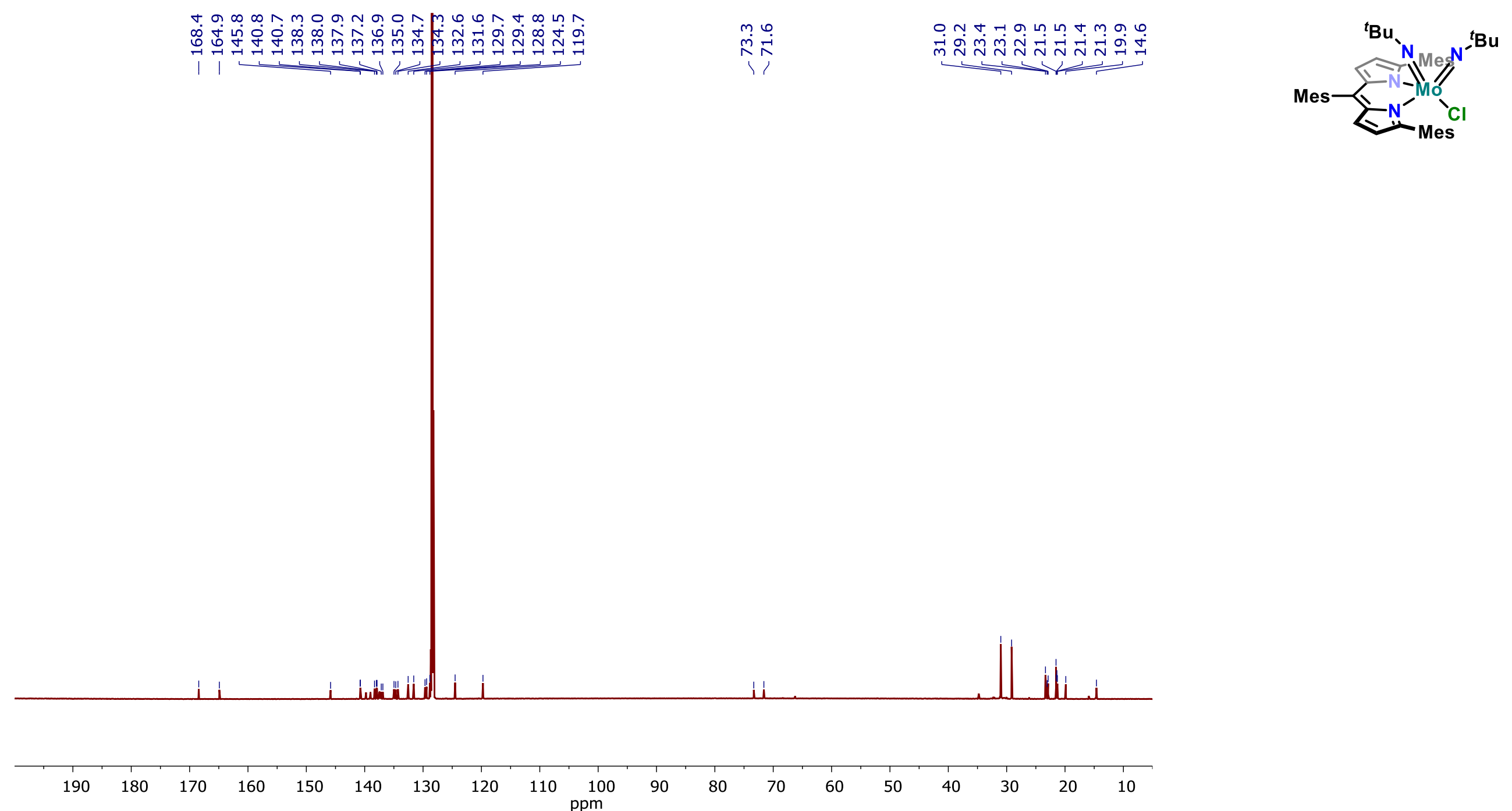

Figure S11: $\quad{ }^{13} \mathrm{C}\left\{{ }^{1} \mathrm{H}\right\}$ NMR spectrum of 1 in $\mathrm{C}_{6} \mathrm{D}_{6}$ at $303 \mathrm{~K}$. 

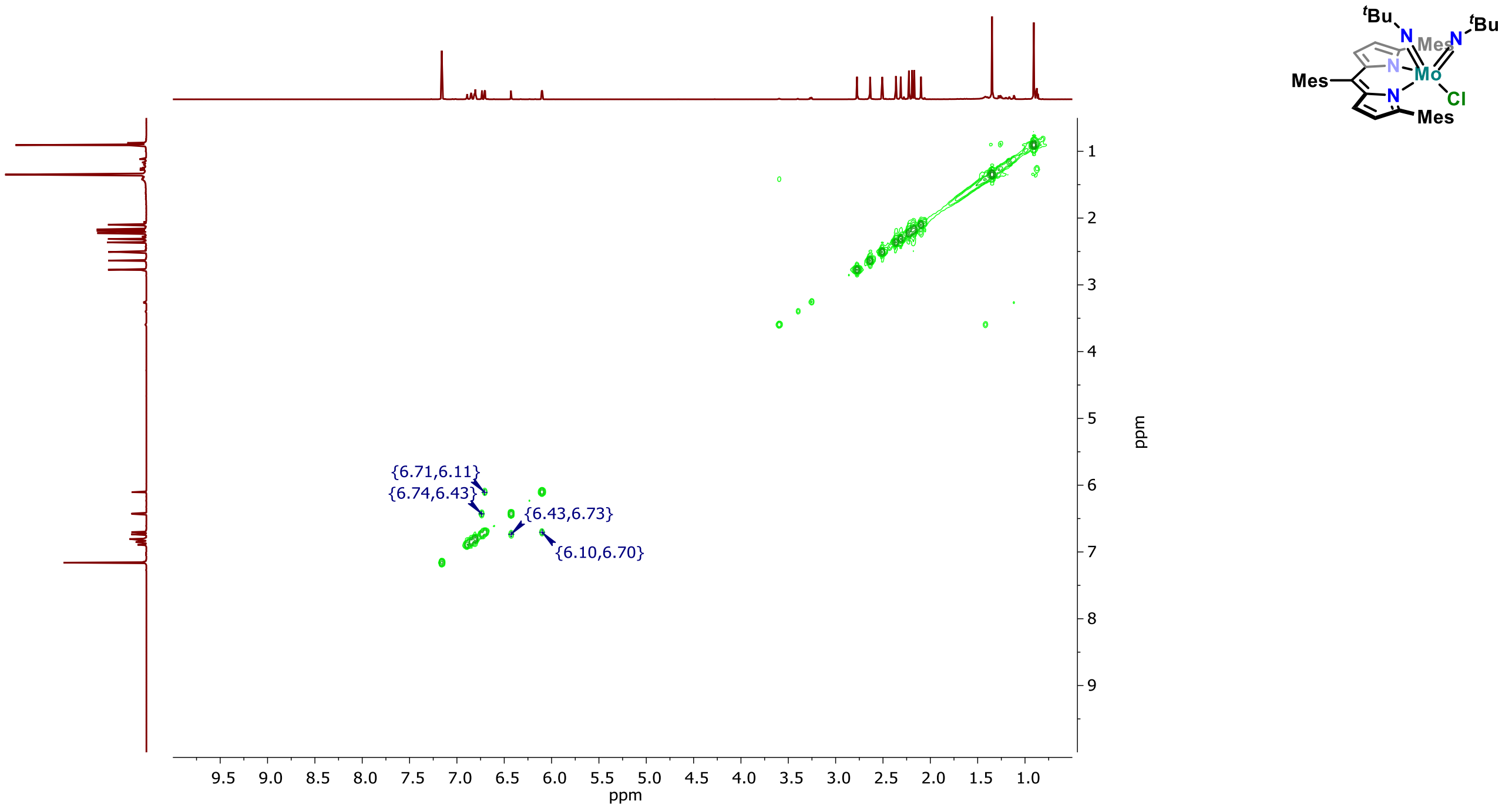

Figure S12: $\quad{ }^{1} \mathrm{H}-{ }^{1} \mathrm{H}$ COSY NMR spectrum of 1 in $\mathrm{C}_{6} \mathrm{D}_{6}$ at $303 \mathrm{~K}$. 

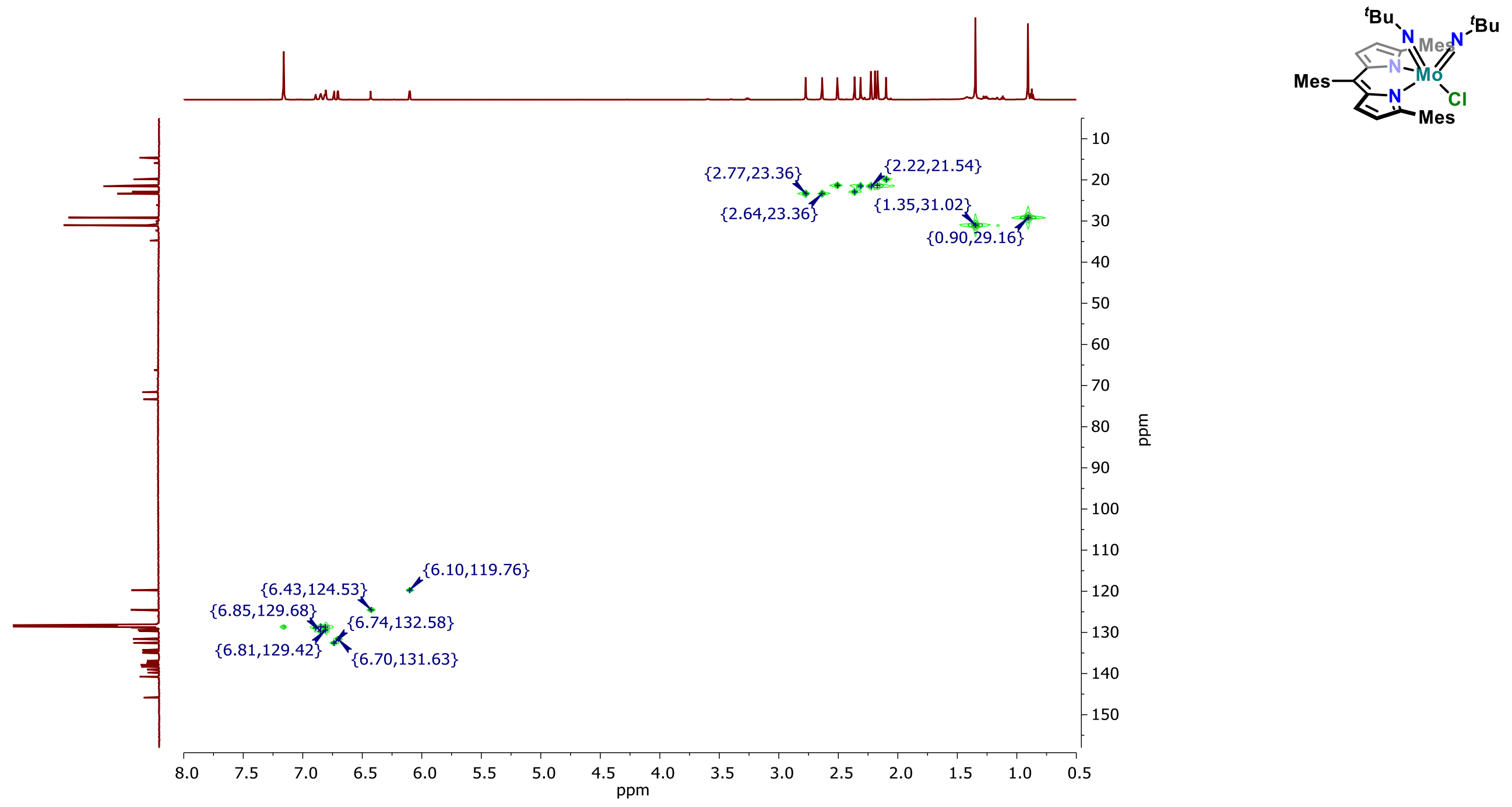

Figure S13: $\quad{ }^{1} \mathrm{H}-{ }^{13} \mathrm{C}$ HSQC NMR spectrum of 1 in $\mathrm{C}_{6} \mathrm{D}_{6}$ at $303 \mathrm{~K}$. 

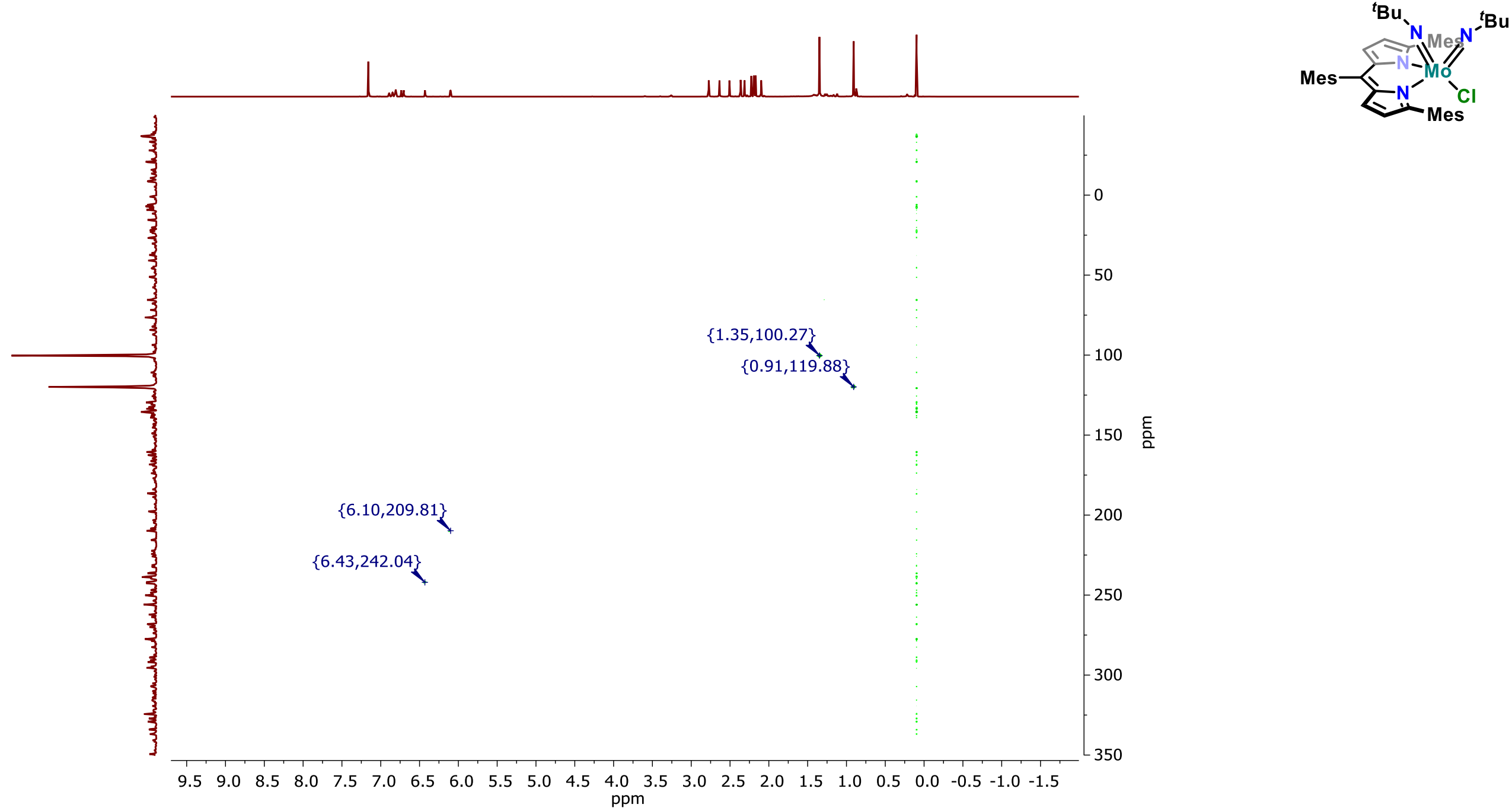

Figure S14: $\quad{ }^{1} \mathrm{H}-{ }^{15} \mathrm{~N}$ HMBC NMR spectrum of 1 in $\mathrm{C}_{6} \mathrm{D}_{6}$ at $303 \mathrm{~K}$. 

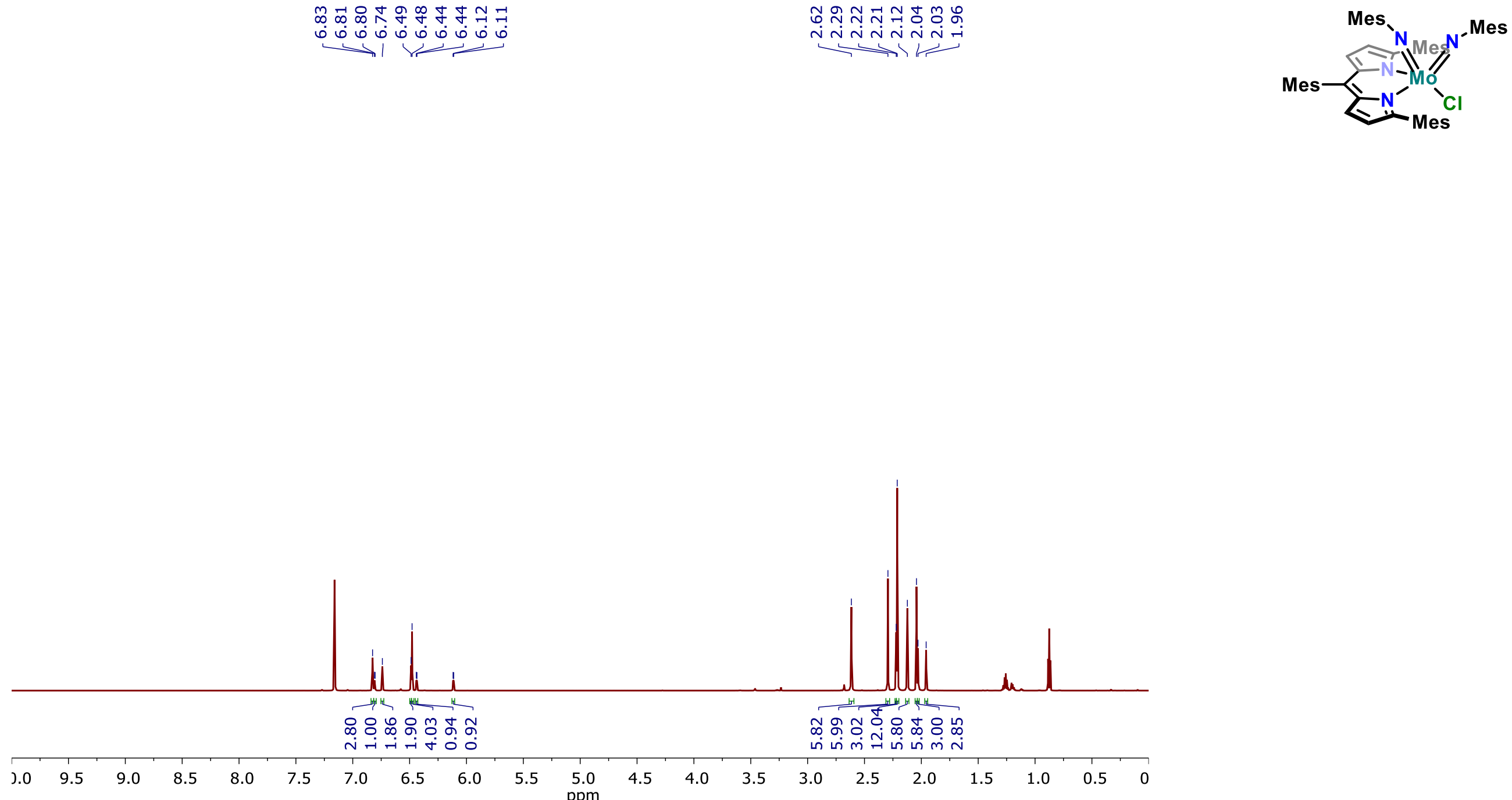

Figure S15: $\quad{ }^{1} \mathrm{H}$ NMR spectrum of 2 in $\mathrm{C}_{6} \mathrm{D}_{6}$ at $303 \mathrm{~K}$. 

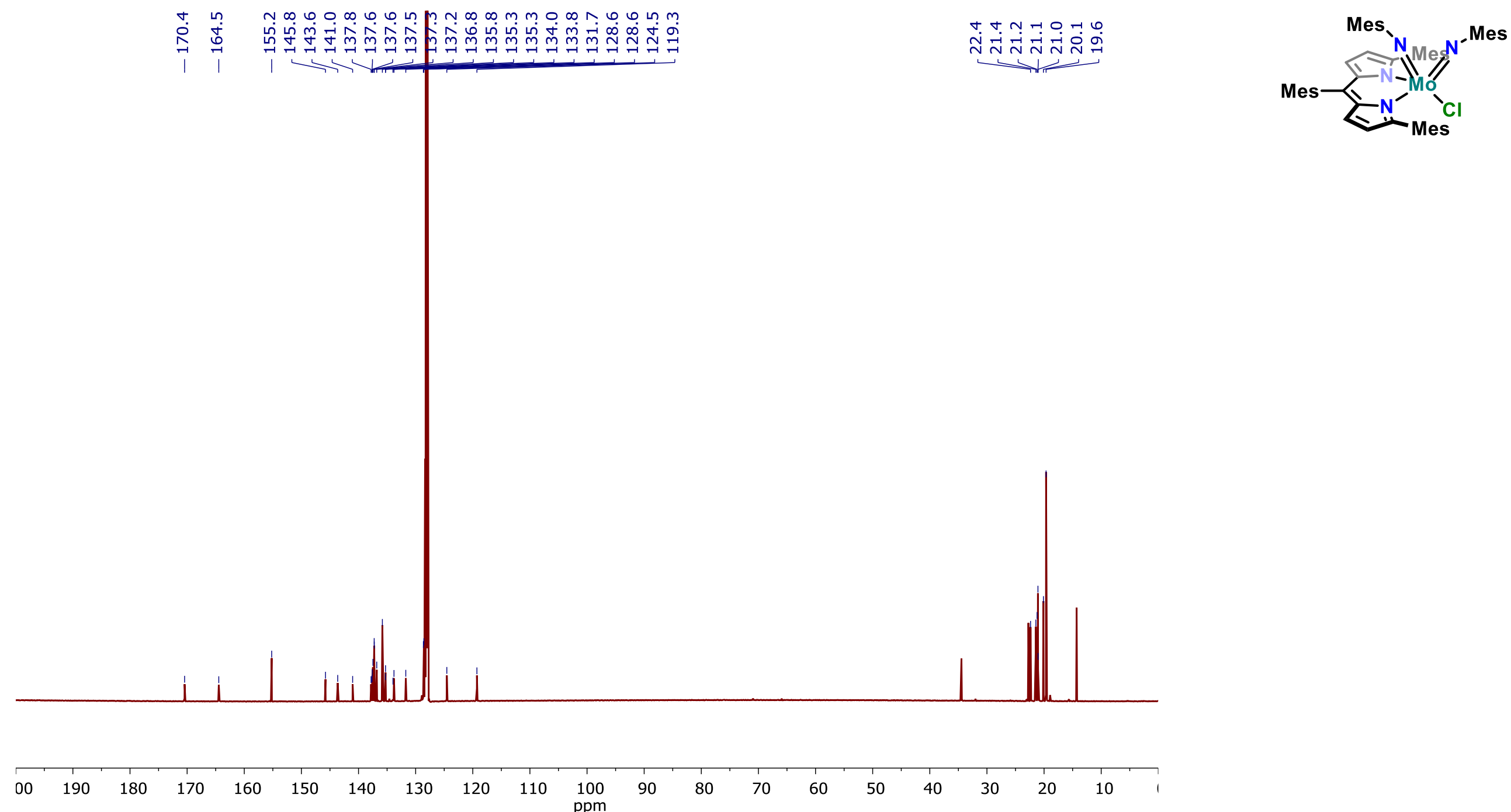

Figure S16: $\quad{ }^{13} \mathrm{C}\left\{{ }^{1} \mathrm{H}\right\}$ NMR spectrum of 2 in $\mathrm{C}_{6} \mathrm{D}_{6}$ at $303 \mathrm{~K}$. 

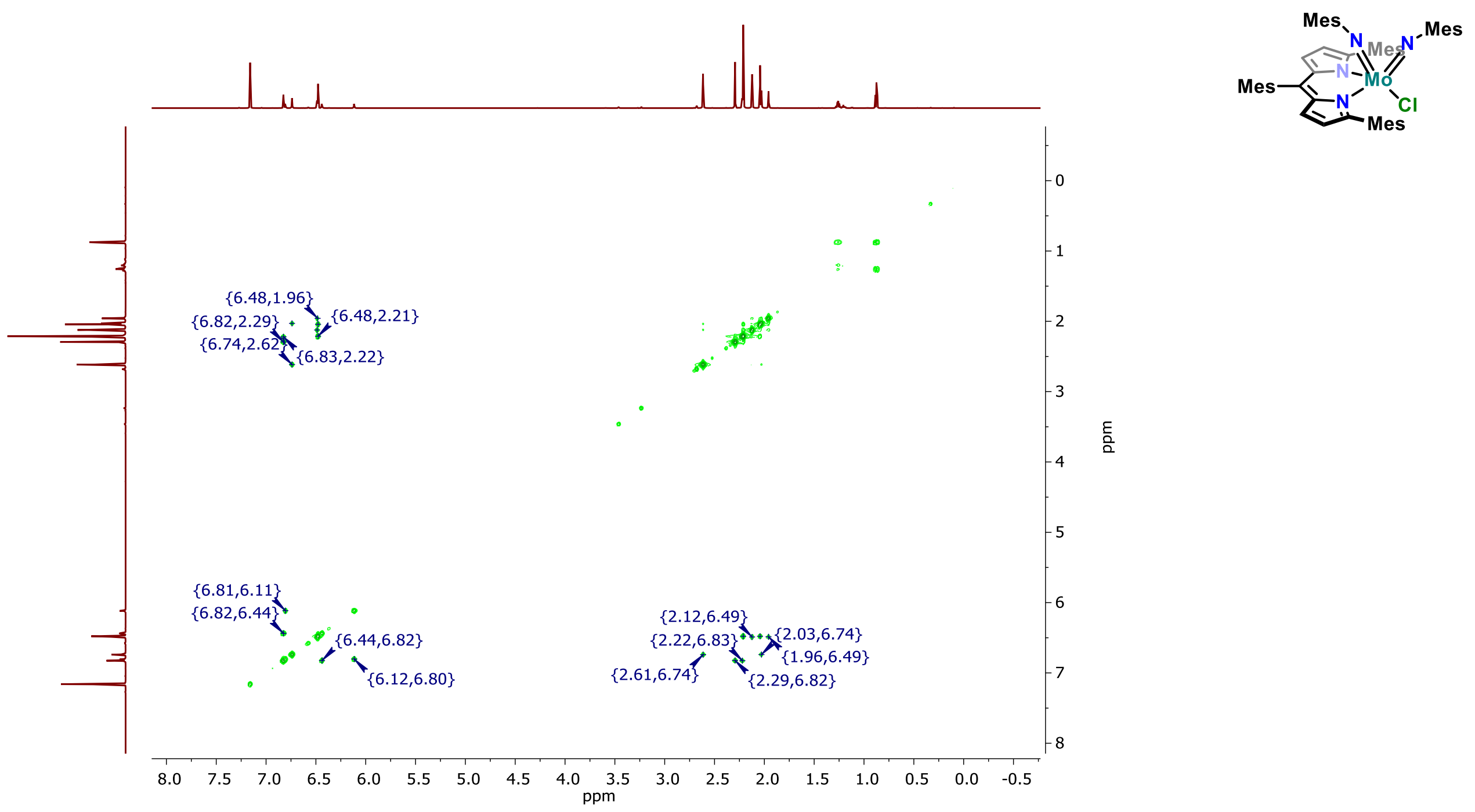

Figure S17: $\quad{ }^{1} \mathrm{H}-{ }^{1} \mathrm{H}$ COSY NMR spectrum of 2 in $\mathrm{C}_{6} \mathrm{D}_{6}$ at $303 \mathrm{~K}$. 

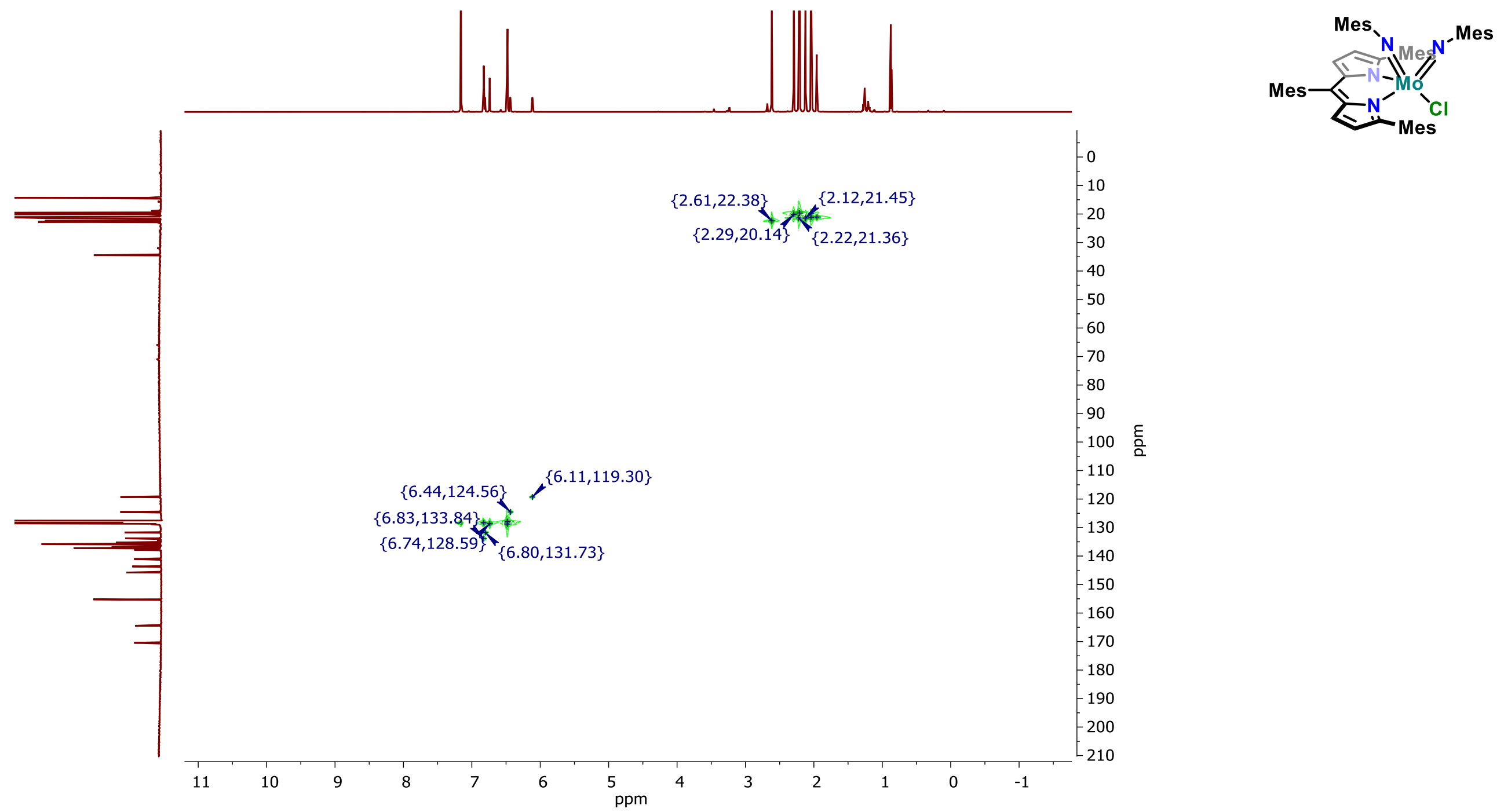

Figure S18: $\quad{ }^{1} \mathrm{H}-{ }^{13} \mathrm{C}$ HSQC NMR spectrum of 2 in $\mathrm{C}_{6} \mathrm{D}_{6}$ at $303 \mathrm{~K}$. 


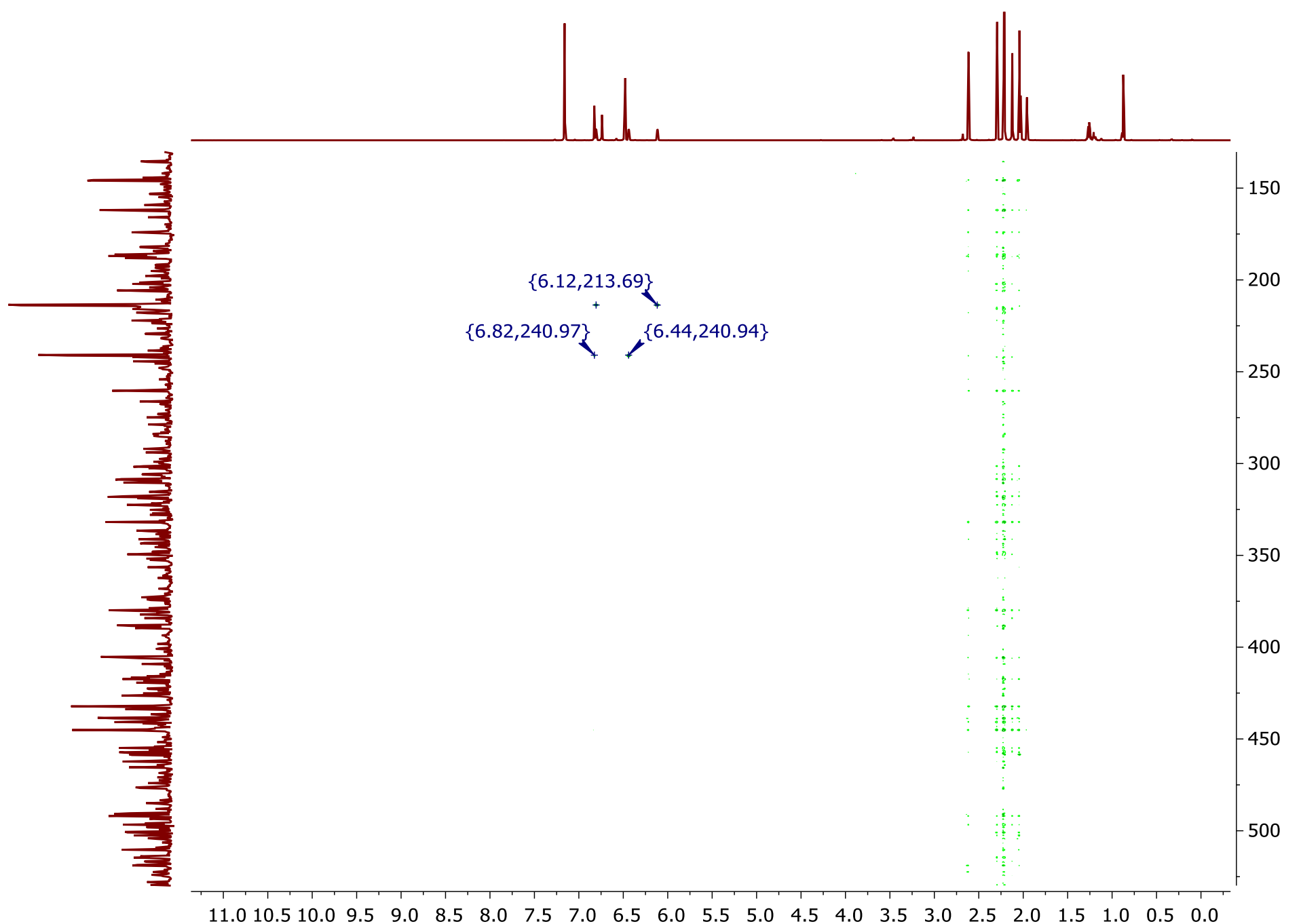

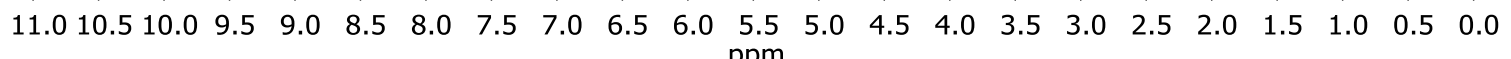

Figure S19: $\quad{ }^{1} \mathrm{H}-{ }^{15} \mathrm{~N}$ HMBC NMR spectrum of 2 in $\mathrm{C}_{6} \mathrm{D}_{6}$ at $303 \mathrm{~K}$. 


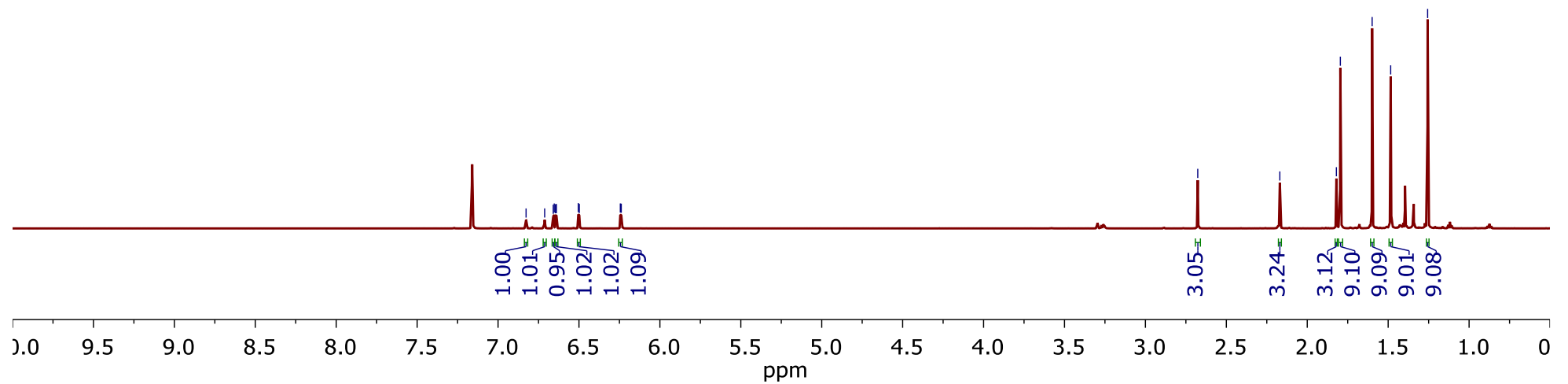

Figure S20: $\quad{ }^{1} \mathrm{H}$ NMR spectrum of 3 in $\mathrm{C}_{6} \mathrm{D}_{6}$ at $303 \mathrm{~K}$. 


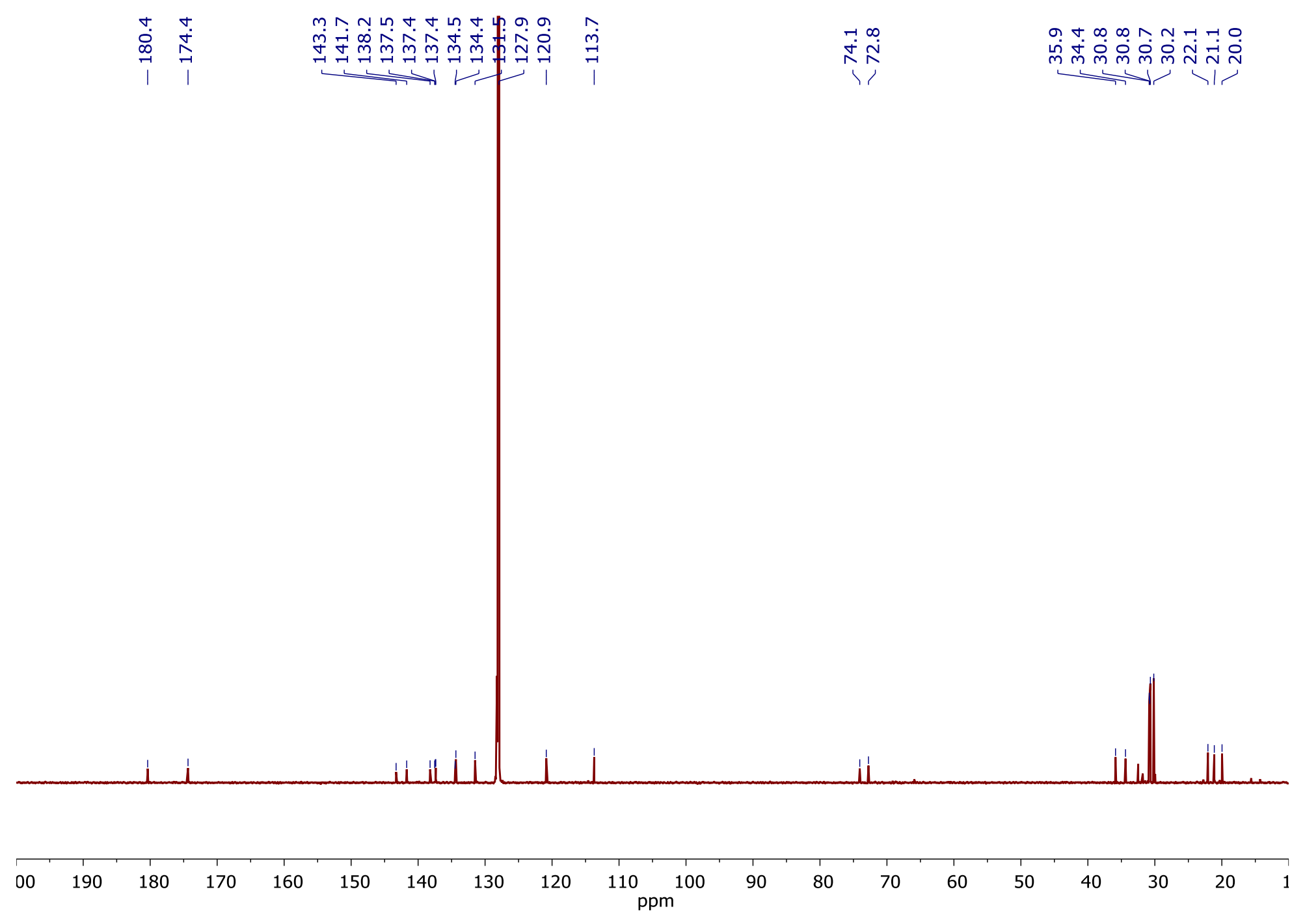

Figure S21: $\quad{ }^{13} \mathrm{C}\left\{{ }^{1} \mathrm{H}\right\}$ NMR spectrum of $\mathbf{3}$ in $\mathrm{C}_{6} \mathrm{D}_{6}$ at $303 \mathrm{~K}$. 

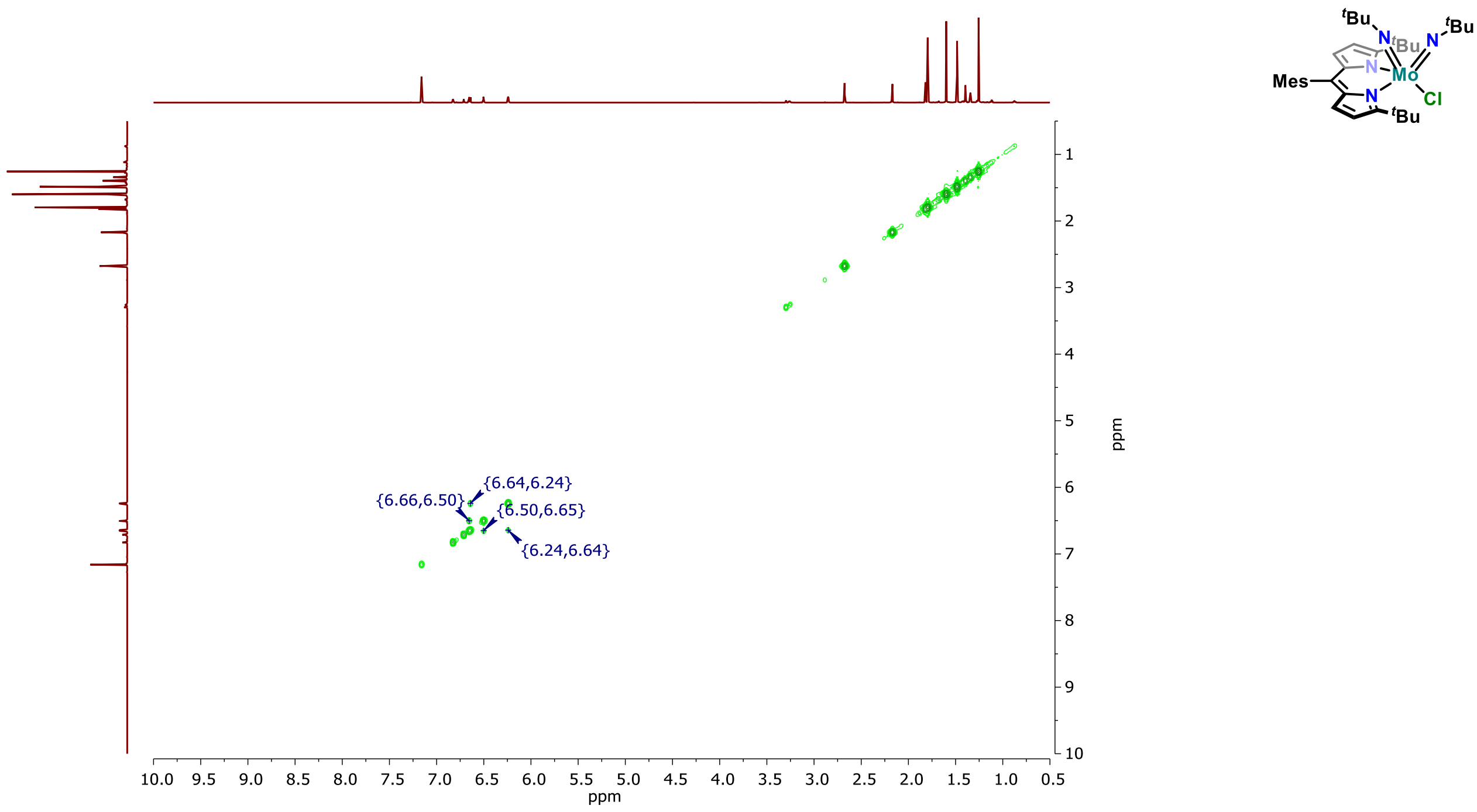

Figure S22: $\quad{ }^{1} \mathrm{H}-{ }^{1} \mathrm{H}$ COSY NMR spectrum of $\mathbf{3}$ in $\mathrm{C}_{6} \mathrm{D}_{6}$ at $303 \mathrm{~K}$. 

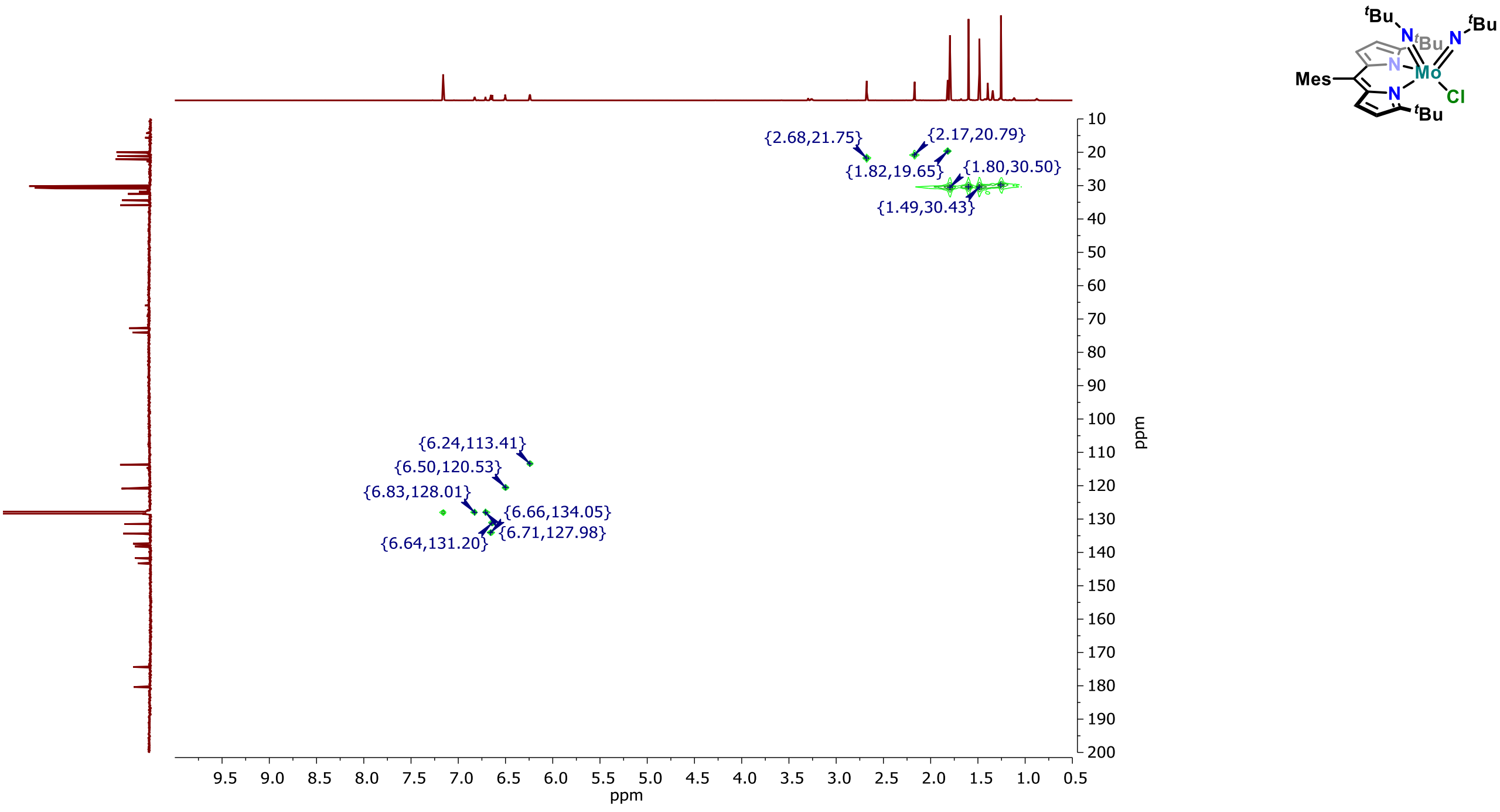

Figure S23: $\quad{ }^{1} \mathrm{H}-{ }^{13} \mathrm{C}$ HSQC NMR spectrum of 3 in $\mathrm{C}_{6} \mathrm{D}_{6}$ at $303 \mathrm{~K}$. 


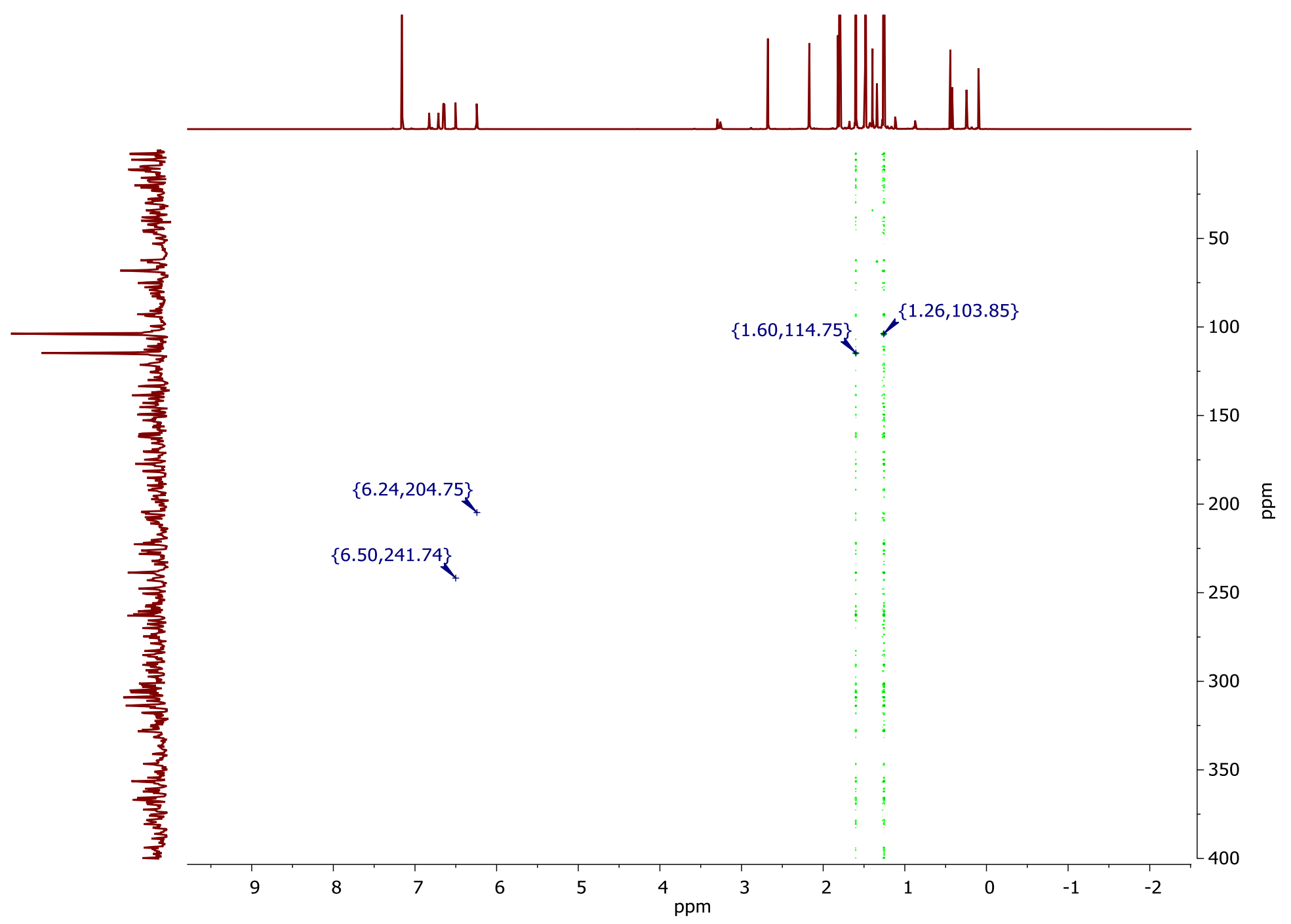

Figure S24: $\quad{ }^{1} \mathrm{H}-{ }^{15} \mathrm{~N}$ HMBC NMR spectrum of 3 in $\mathrm{C}_{6} \mathrm{D}_{6}$ at $303 \mathrm{~K}$. 

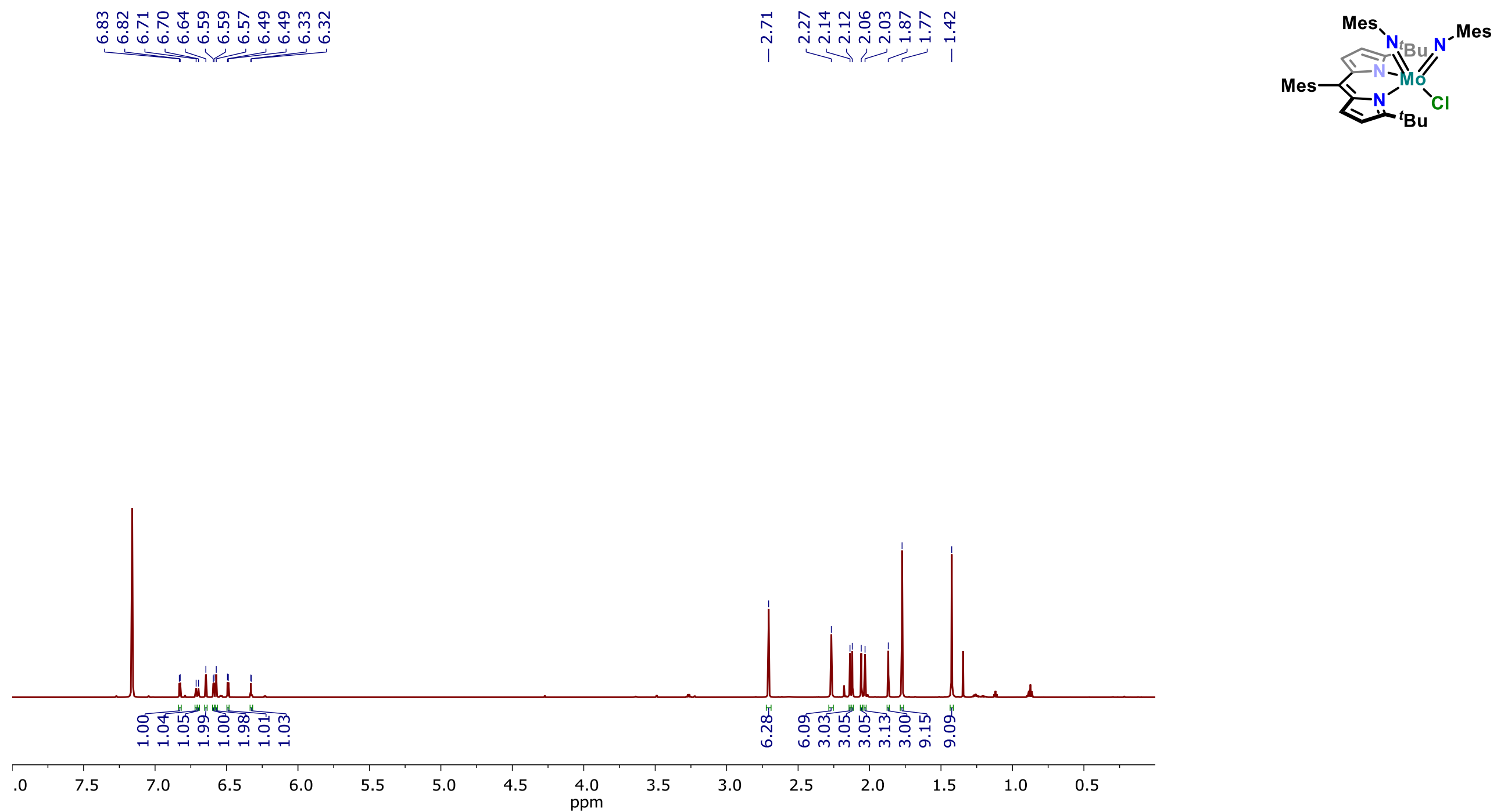

Figure S25: $\quad{ }^{1} \mathrm{H}$ NMR spectrum of 4 in $\mathrm{C}_{6} \mathrm{D}_{6}$ at $303 \mathrm{~K}$. 


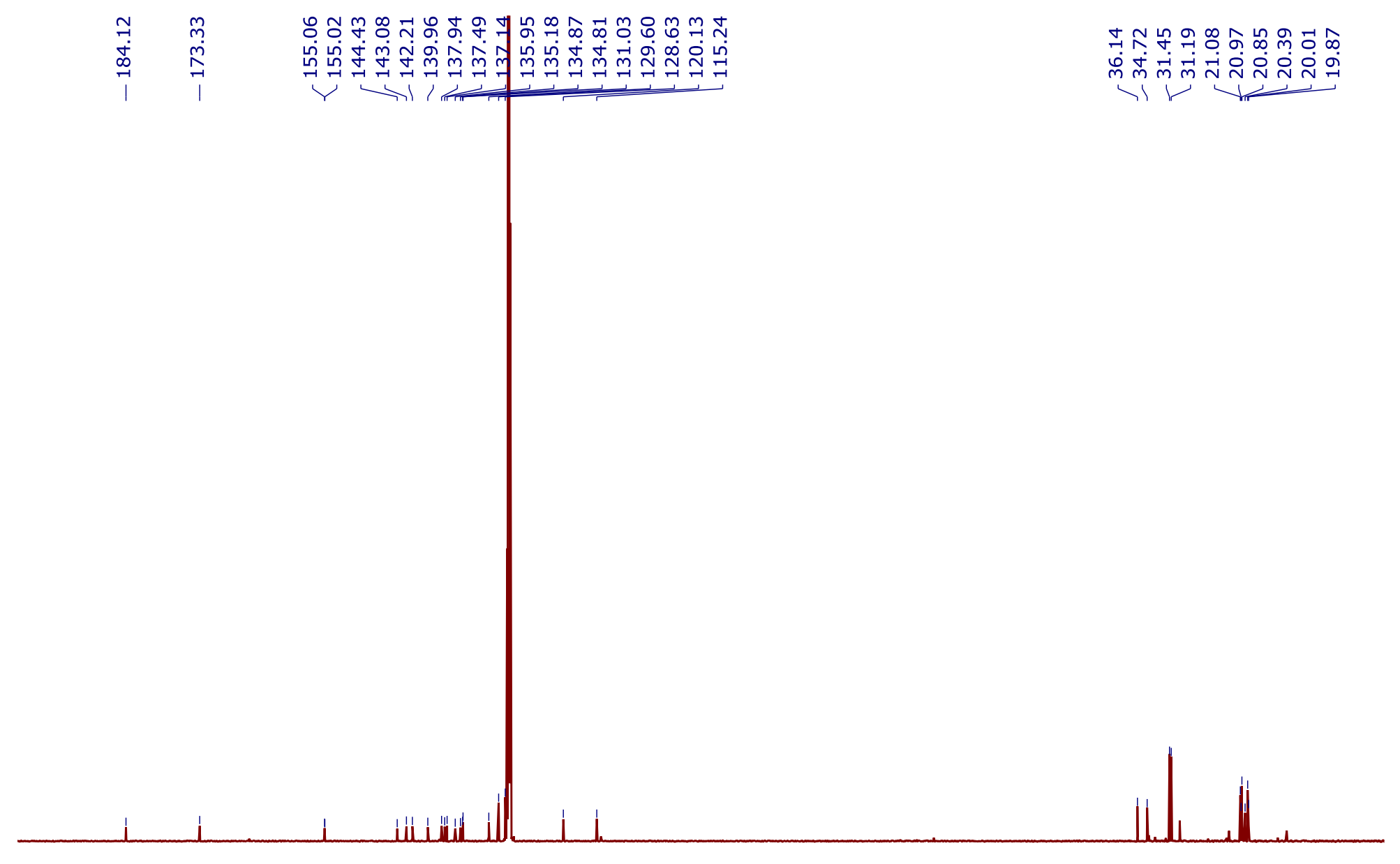

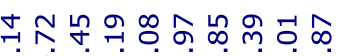



似

Figure S26: $\quad{ }^{13} \mathrm{C}\left\{{ }^{1} \mathrm{H}\right\}$ NMR spectrum of 4 in $\mathrm{C}_{6} \mathrm{D}_{6}$ at $303 \mathrm{~K}$. 

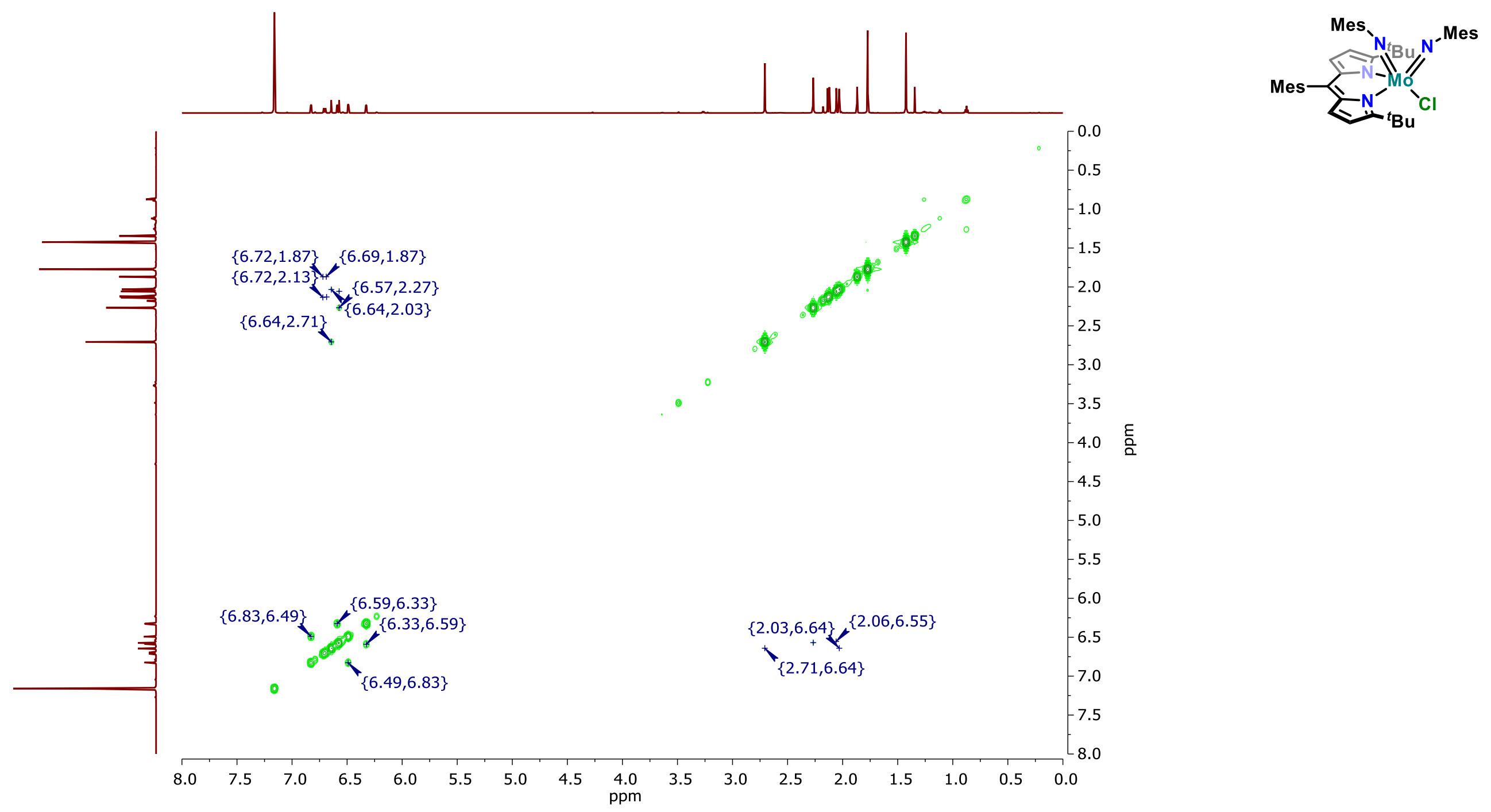

Figure S27: $\quad{ }^{1} \mathrm{H}-{ }^{1} \mathrm{H}$ COSY NMR spectrum of 4 in $\mathrm{C}_{6} \mathrm{D}_{6}$ at $303 \mathrm{~K}$. 

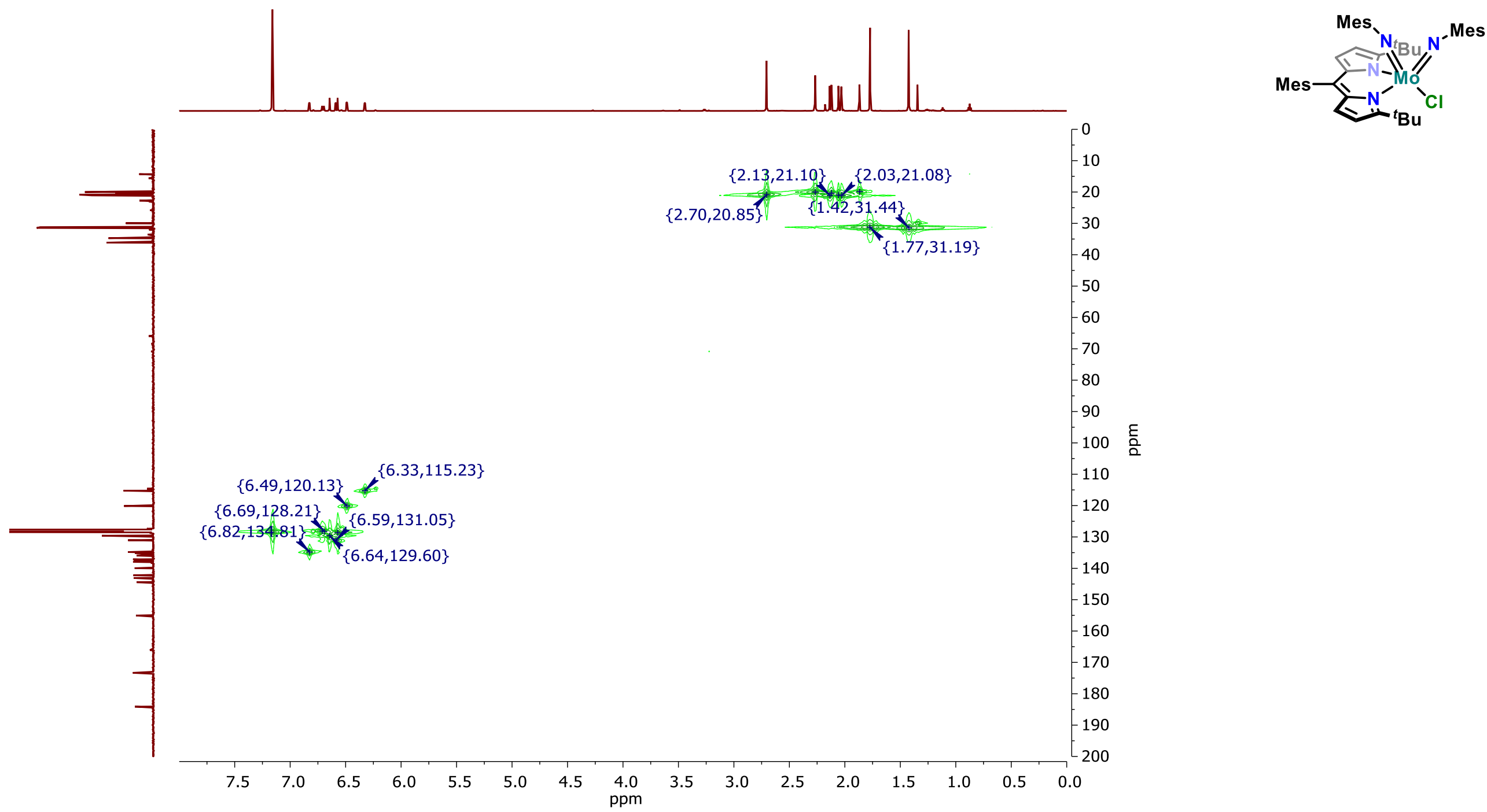

Figure S28: $\quad{ }^{1} \mathrm{H}-{ }^{13} \mathrm{C}$ HSOC NMR spectrum of 4 in $\mathrm{C}_{6} \mathrm{D}_{6}$ at $303 \mathrm{~K}$. 


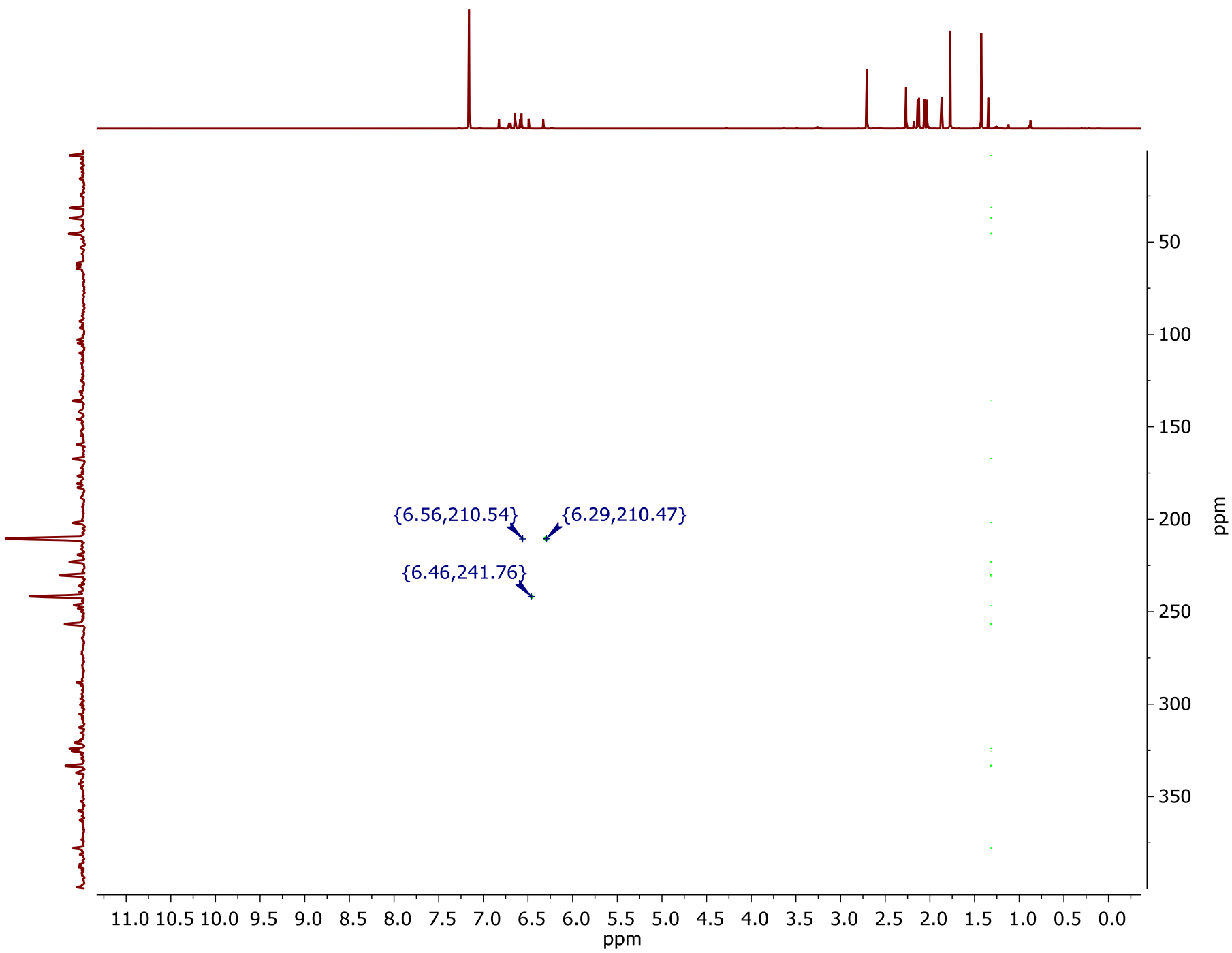

Figure S29: $\quad{ }^{1} \mathrm{H}-{ }^{15} \mathrm{~N}$ HMBC NMR spectrum of 4 in $\mathrm{C}_{6} \mathrm{D}_{6}$ at $303 \mathrm{~K}$. 

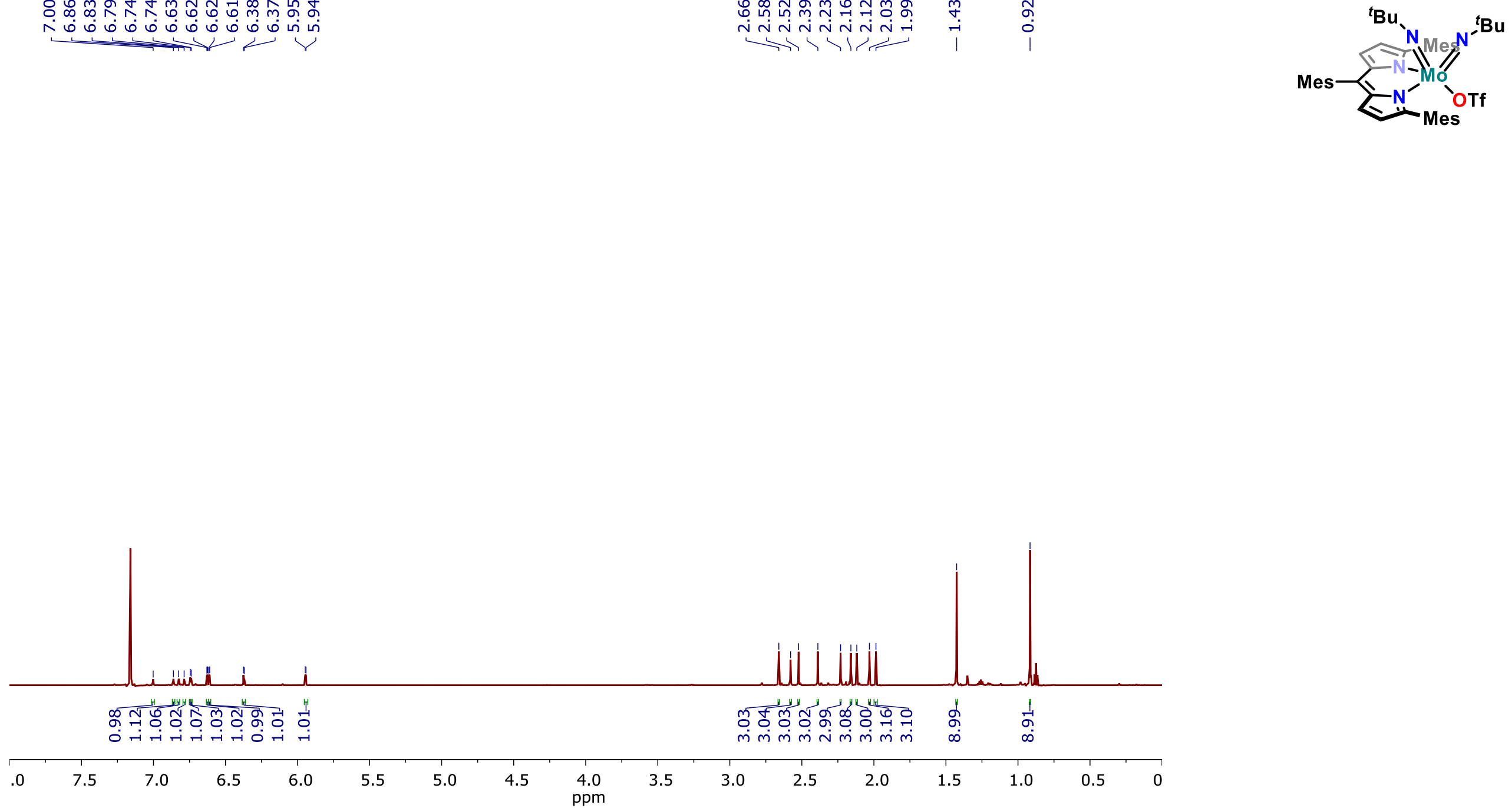

Figure S30: $\quad{ }^{1} \mathrm{H}$ NMR spectrum of 6 in $\mathrm{C}_{6} \mathrm{D}_{6}$ at $303 \mathrm{~K}$. 

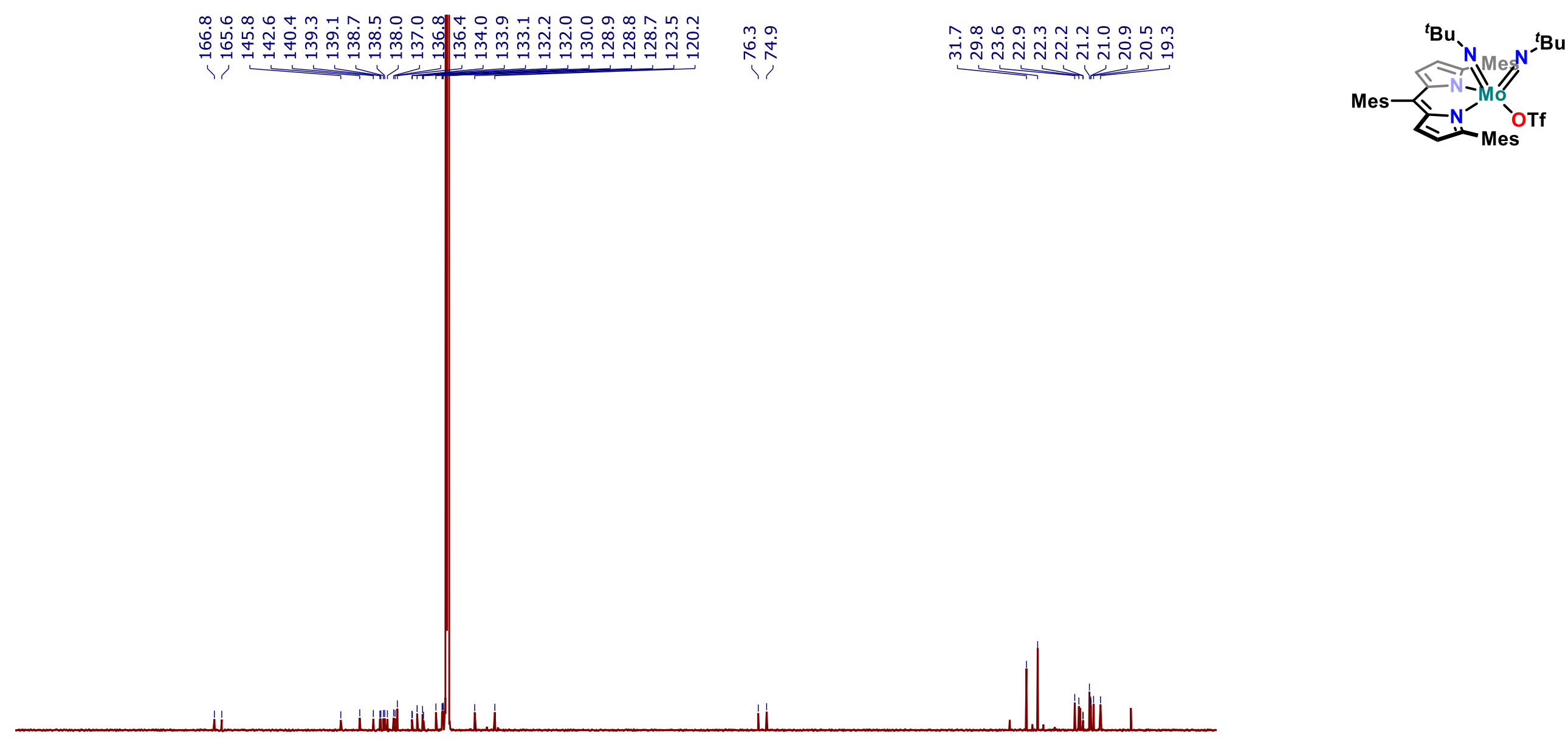

$190 \quad 180 \quad 170 \quad 160$

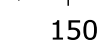

$140 \quad 130$

120

Figure S31: $\quad{ }^{13} \mathrm{C}\left\{{ }^{1} \mathrm{H}\right\}$ NMR spectrum of 6 in $\mathrm{C}_{6} \mathrm{D}_{6}$ at $303 \mathrm{~K}$. 

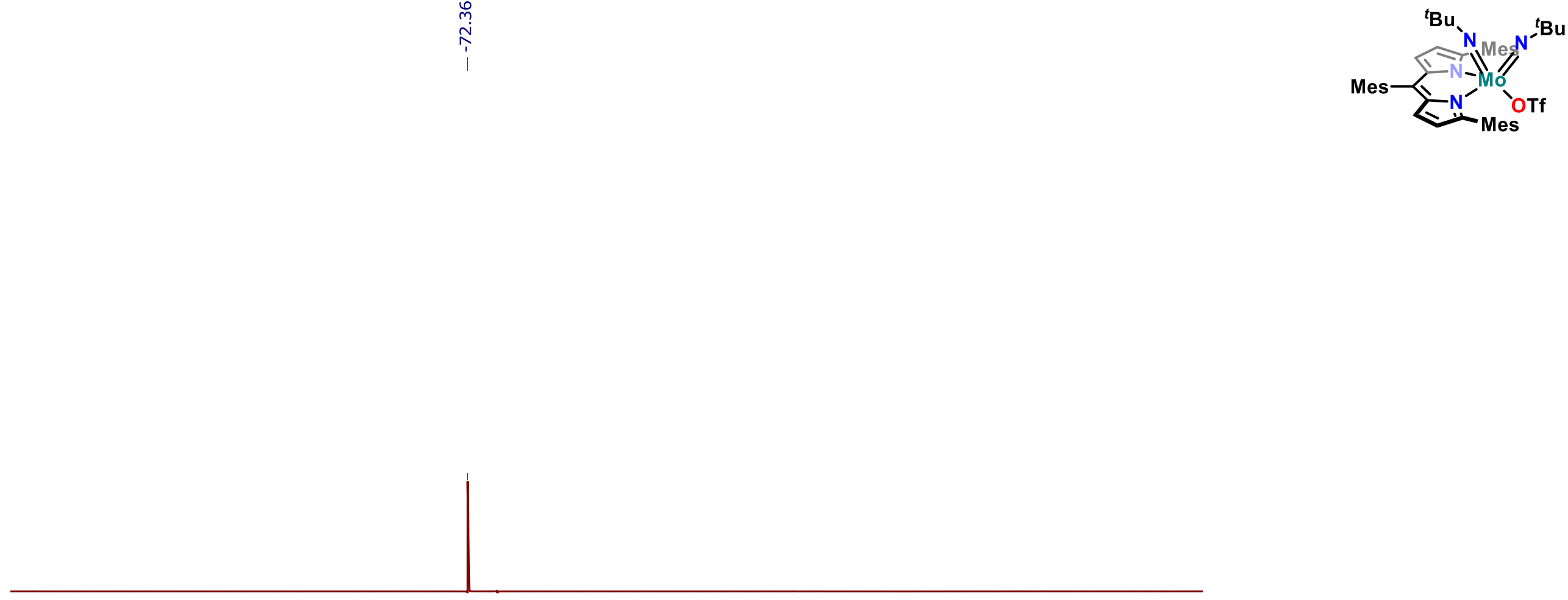

$\begin{array}{lllllllllllllllllllllllllllll}10 & 0 & -10 & -20 & -30 & -40 & -50 & -60 & -70 & -80 & -90 & -100 & -110 & -120 & -130 & -140 & -150 & -160 & -170 & -180 & -190 & -200 & -210\end{array}$

Figure S32: $\quad{ }^{19} \mathrm{~F}$ NMR spectrum of 6 in $\mathrm{C}_{6} \mathrm{D}_{6}$ at $303 \mathrm{~K}$. 

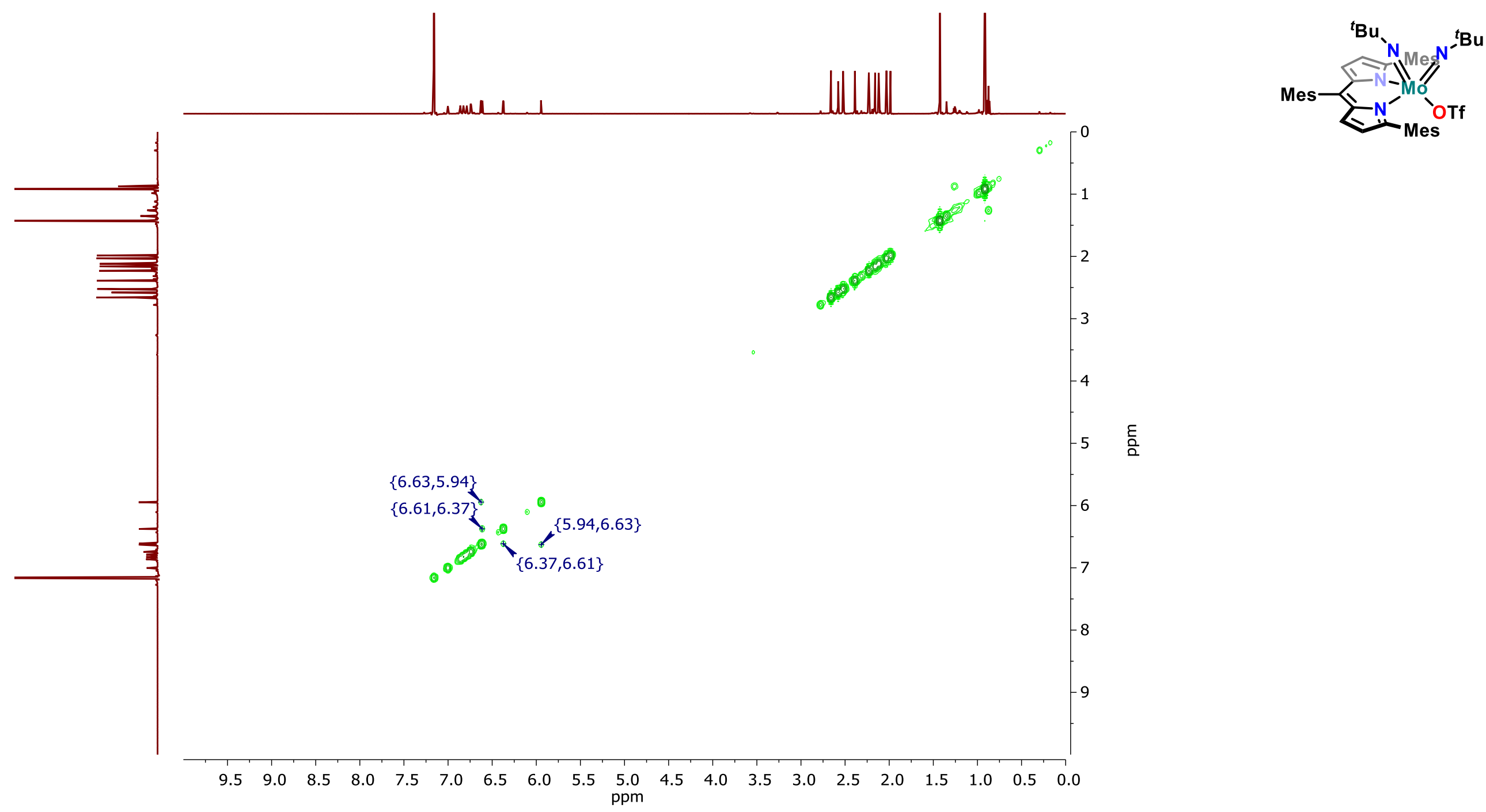

Figure S33: $\quad{ }^{1} \mathrm{H}-{ }^{1} \mathrm{H}$ COSY NMR spectrum of 6 in $\mathrm{C}_{6} \mathrm{D}_{6}$ at $303 \mathrm{~K}$. 

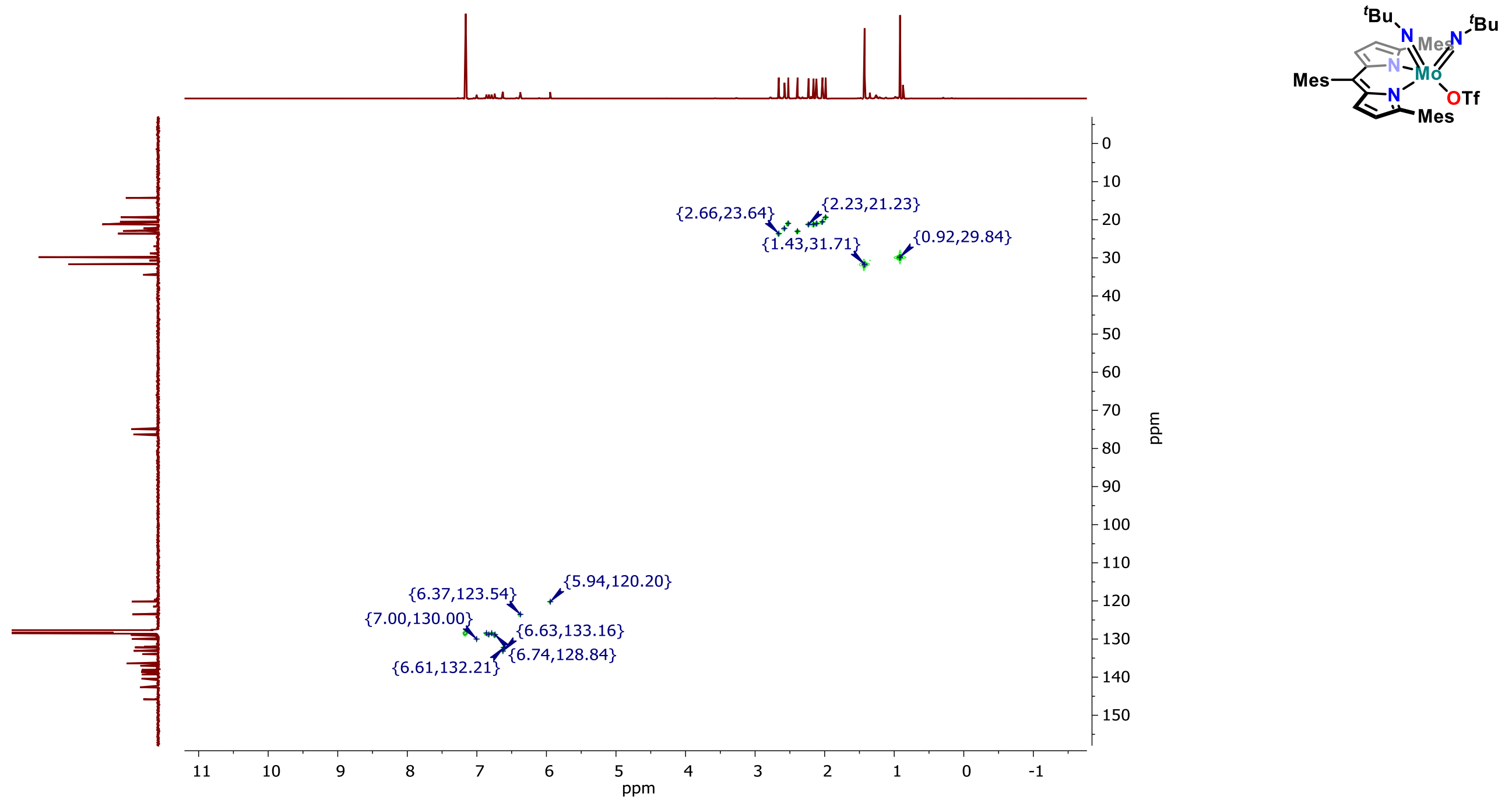

Figure S34: $\quad{ }^{1} \mathrm{H}-{ }^{13} \mathrm{C}$ HSQC NMR spectrum of 6 in $\mathrm{C}_{6} \mathrm{D}_{6}$ at $303 \mathrm{~K}$. 

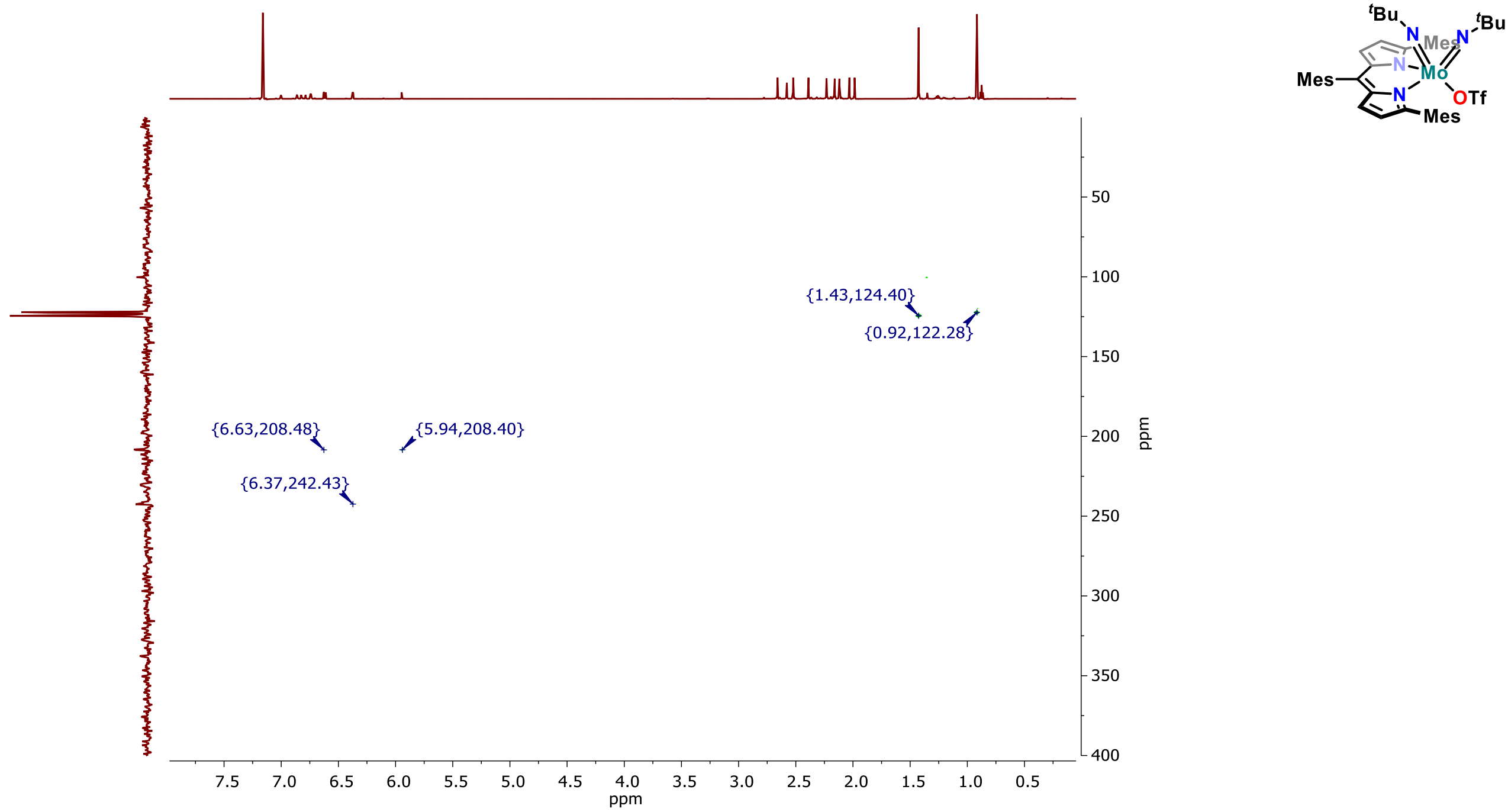

Figure S35: $\quad{ }^{1} \mathrm{H}-{ }^{15} \mathrm{~N}$ HMBC NMR spectrum of 6 in $\mathrm{C}_{6} \mathrm{D}_{6}$ at $303 \mathrm{~K}$. 


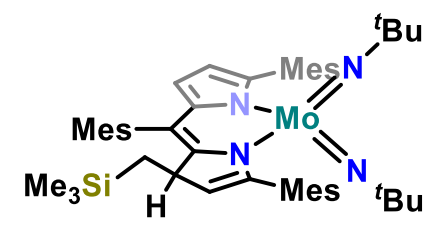

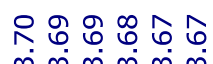

mimm

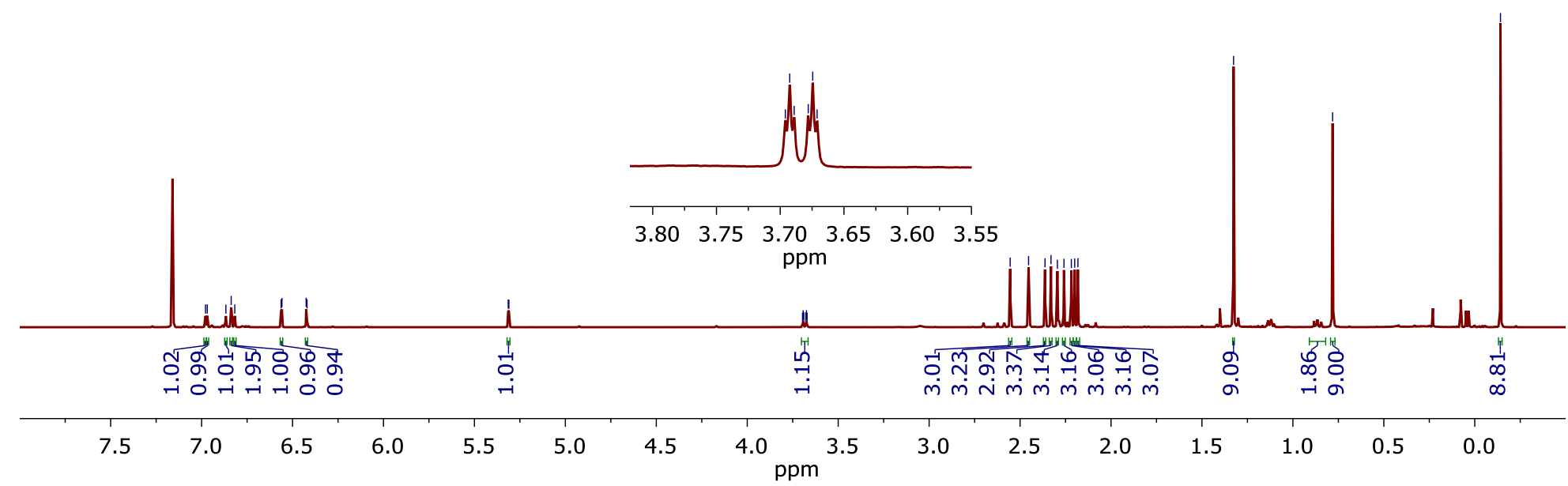

Figure S36: $\quad{ }^{1} \mathrm{H}$ NMR spectrum of 7 in $\mathrm{C}_{6} \mathrm{D}_{6}$ at $303 \mathrm{~K}$, with a detail of the doublet of triplets observed at $\delta 3.69 \mathrm{ppm}$. 

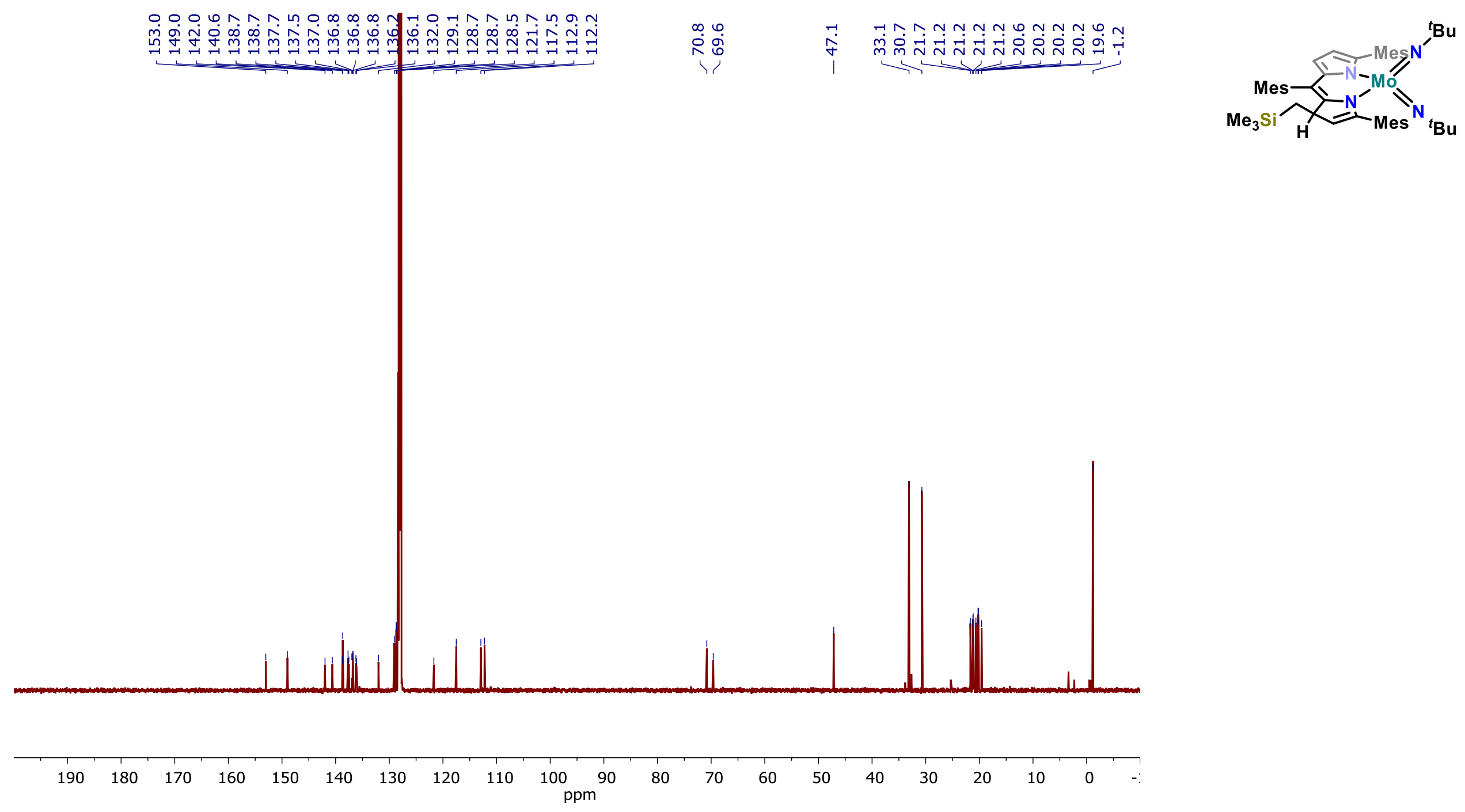

Figure S37: $\quad{ }^{13} \mathrm{C}\left\{{ }^{1} \mathrm{H}\right\}$ NMR spectrum of 7 in $\mathrm{C}_{6} \mathrm{D}_{6}$ at $303 \mathrm{~K}$. 

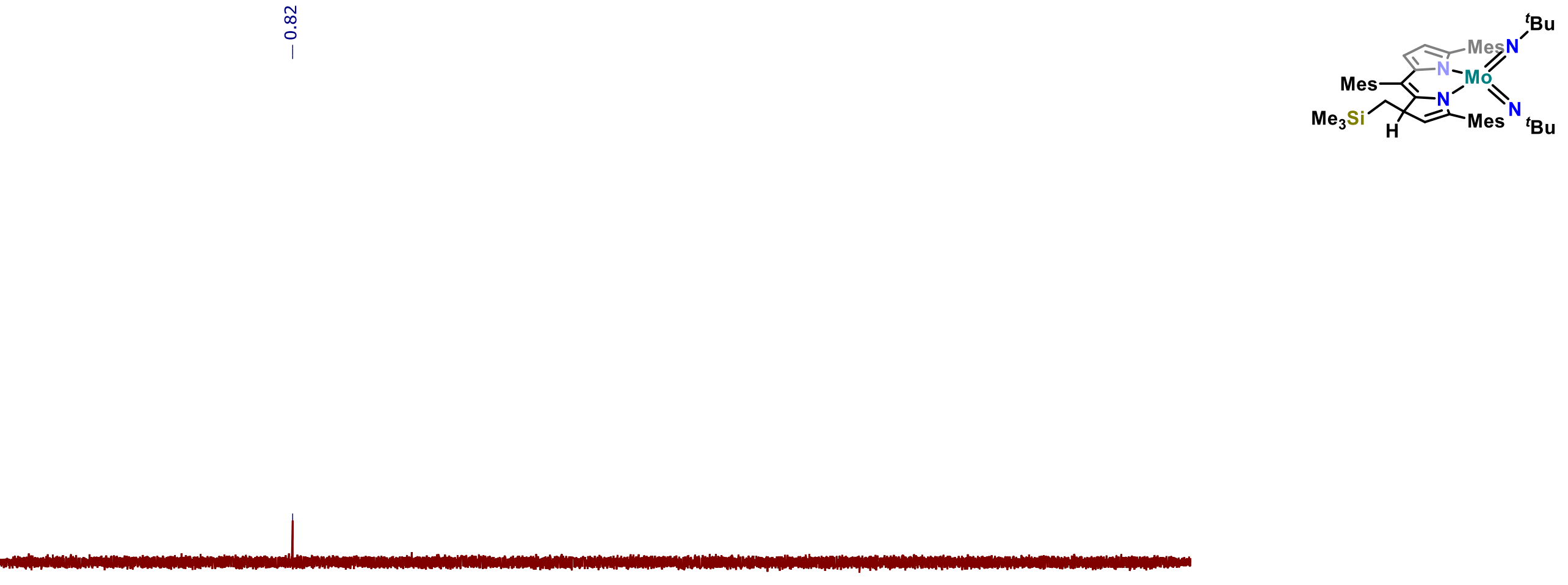

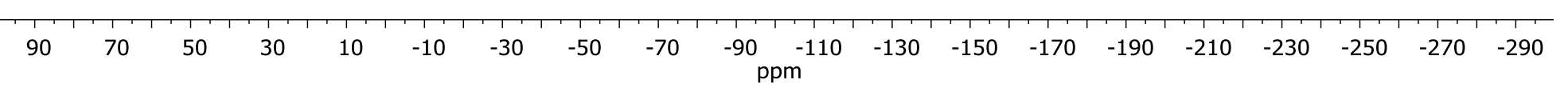

Figure S38: $\quad{ }^{29} \mathrm{Si}\left\{{ }^{1} \mathrm{H}\right\}$ NMR spectrum of 7 in $\mathrm{C}_{6} \mathrm{D}_{6}$ at $303 \mathrm{~K}$. 

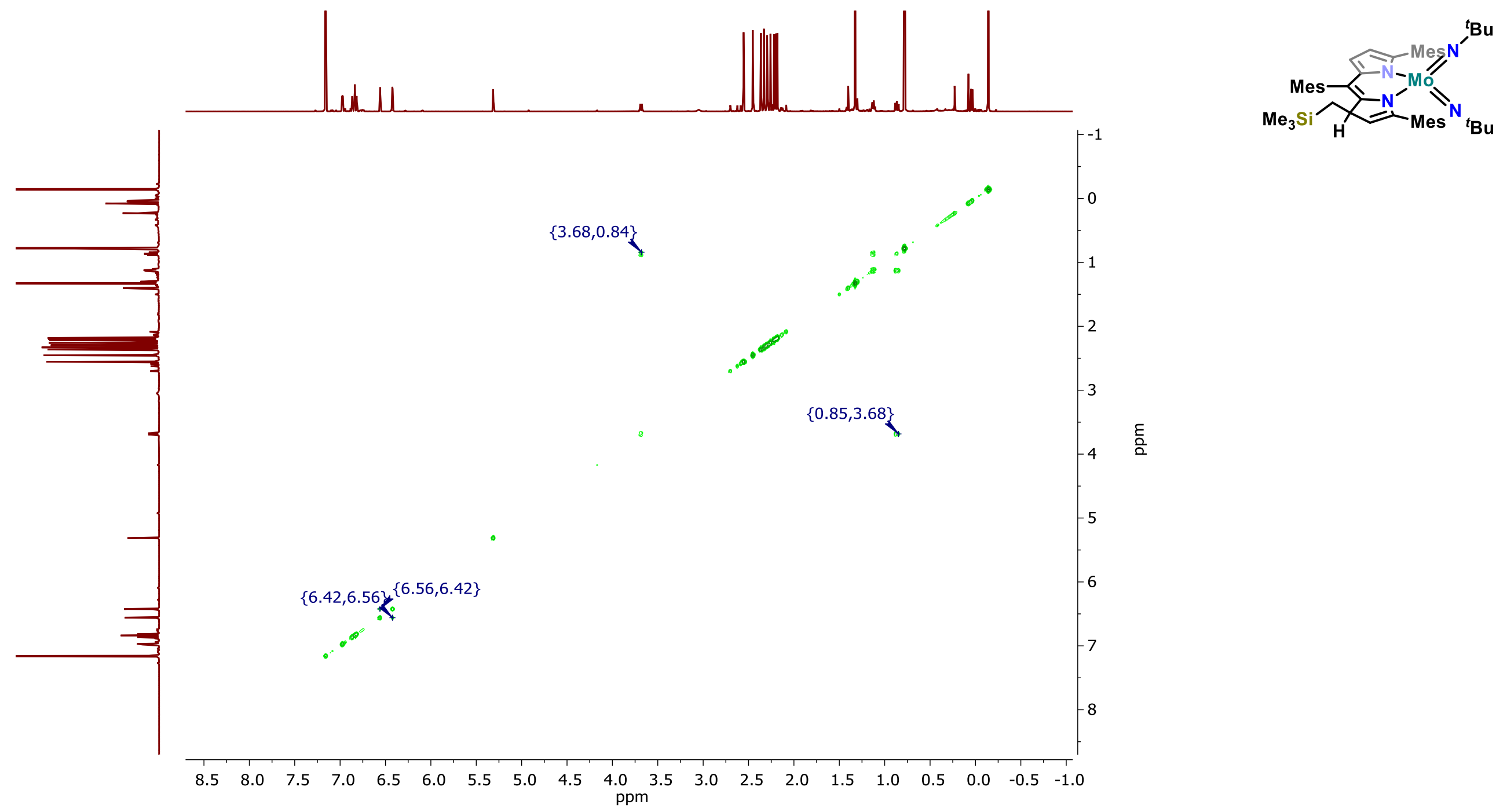

Figure S39: $\quad{ }^{1} \mathrm{H}-{ }^{1} \mathrm{H}$ COSY NMR spectrum of 7 in $\mathrm{C}_{6} \mathrm{D}_{6}$ at $303 \mathrm{~K}$. 

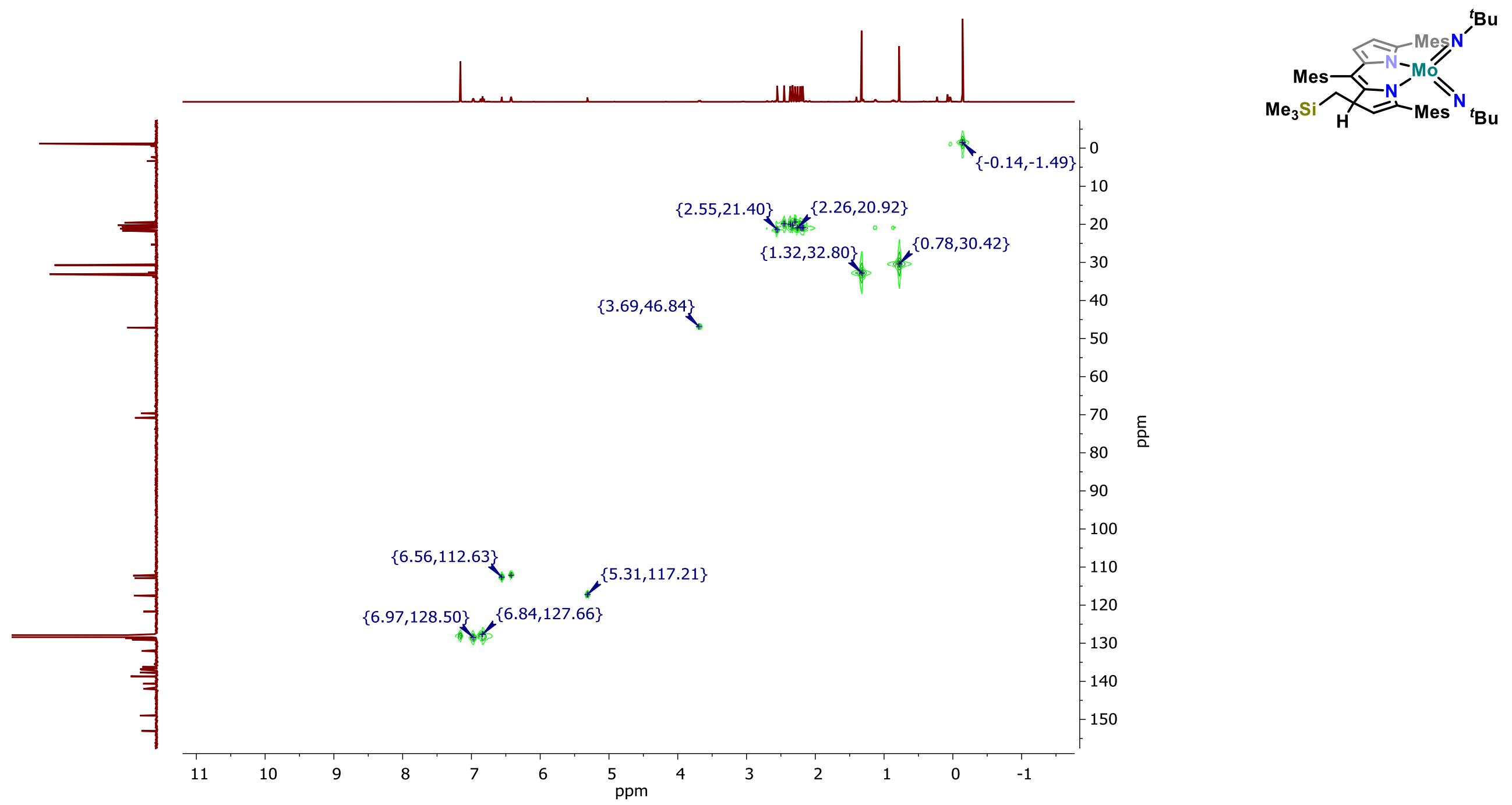

Figure S40: $\quad{ }^{1} \mathrm{H}-{ }^{13} \mathrm{C} \mathrm{HSQC} \mathrm{NMR} \mathrm{spectrum} \mathrm{of} 7$ in $\mathrm{C}_{6} \mathrm{D}_{6}$ at $303 \mathrm{~K}$. 

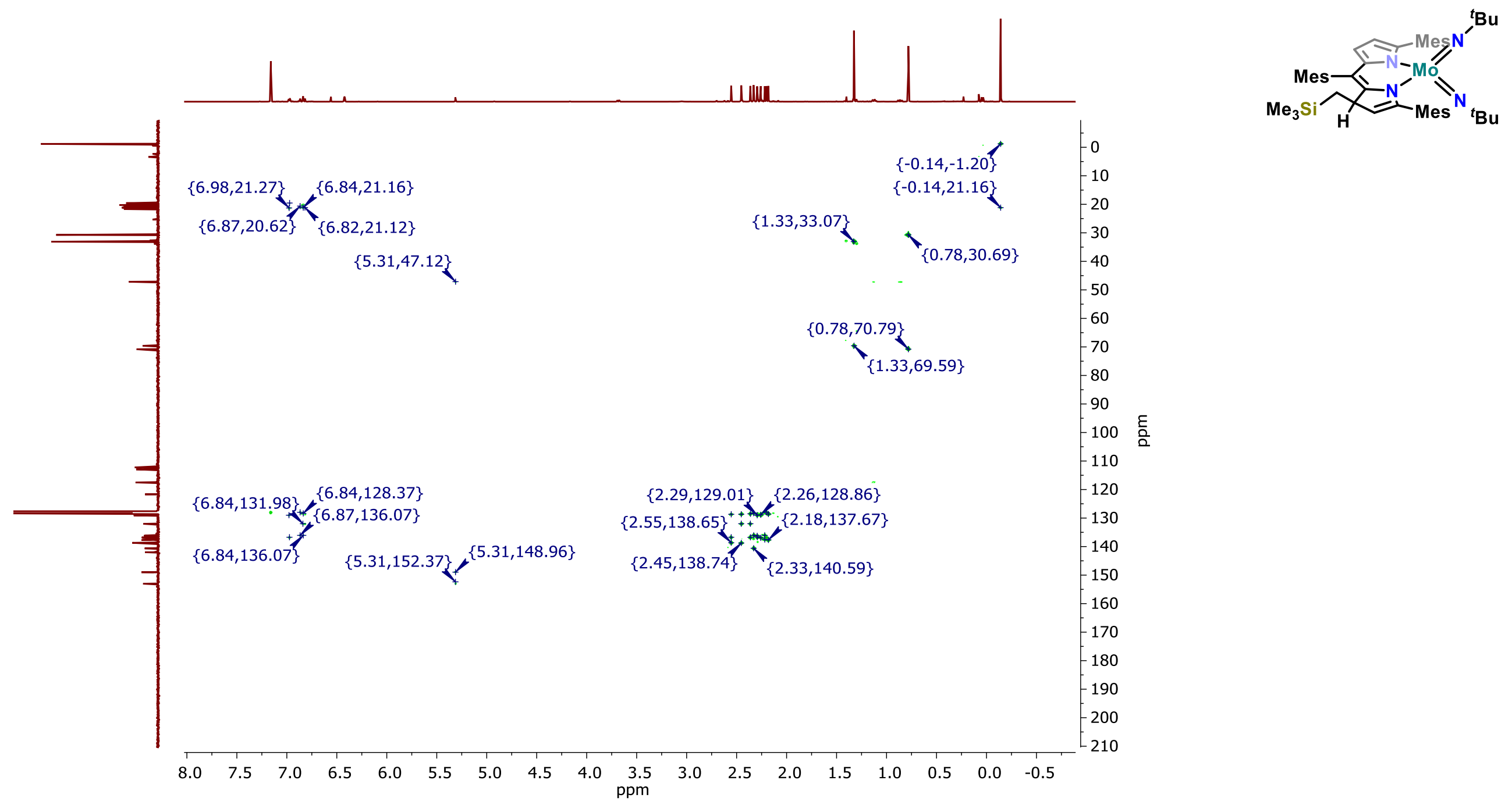

Figure S41: $\quad{ }^{1} \mathrm{H}-{ }^{13} \mathrm{C}$ HMBC NMR spectrum of 7 in $\mathrm{C}_{6} \mathrm{D}_{6}$ at $303 \mathrm{~K}$. 

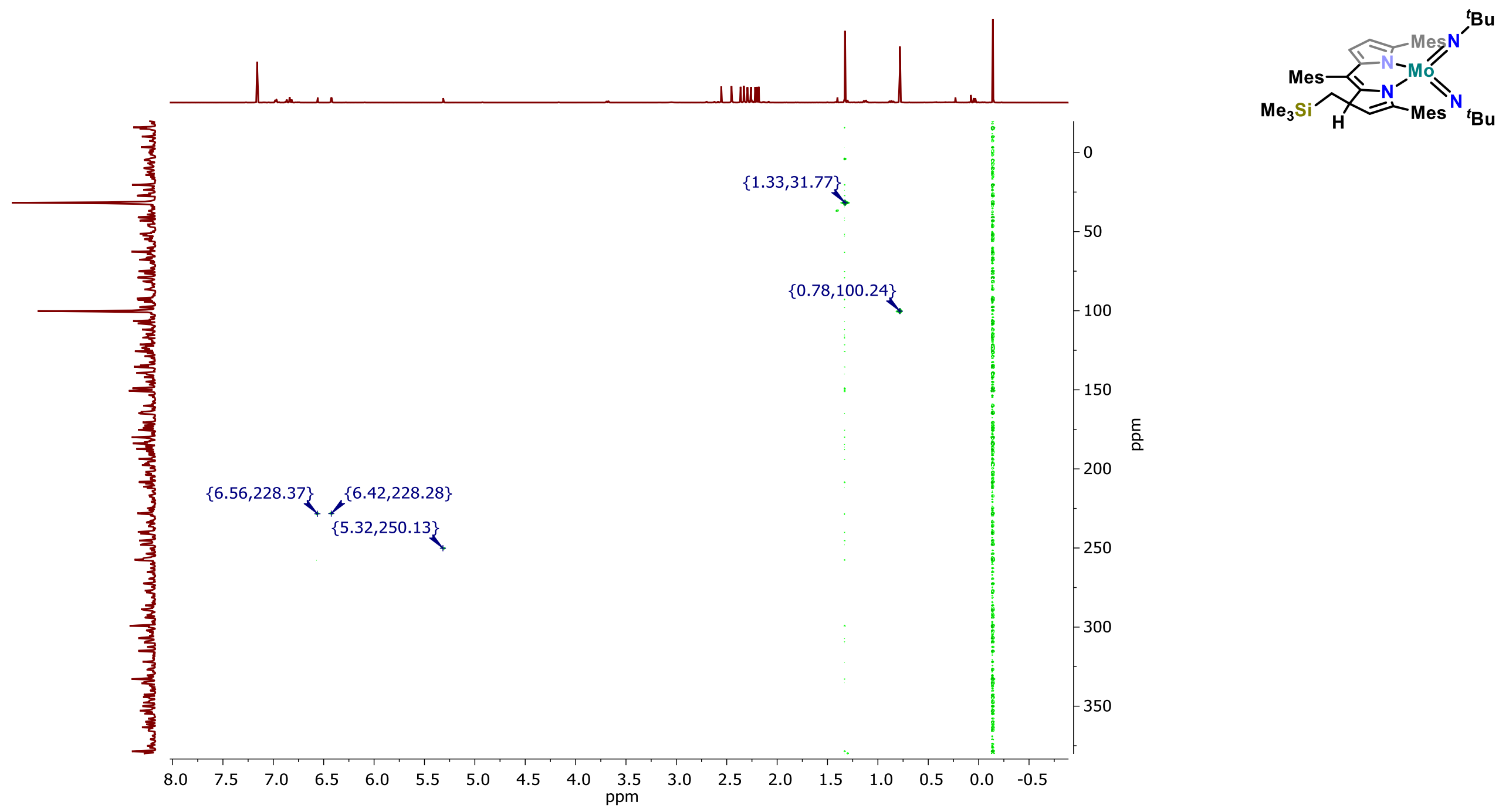

Figure S42: $\quad{ }^{1} \mathrm{H}-{ }^{15} \mathrm{~N}$ HMBC NMR spectrum of 7 in $\mathrm{C}_{6} \mathrm{D}_{6}$ at $303 \mathrm{~K}$. 
3. UV-Vis

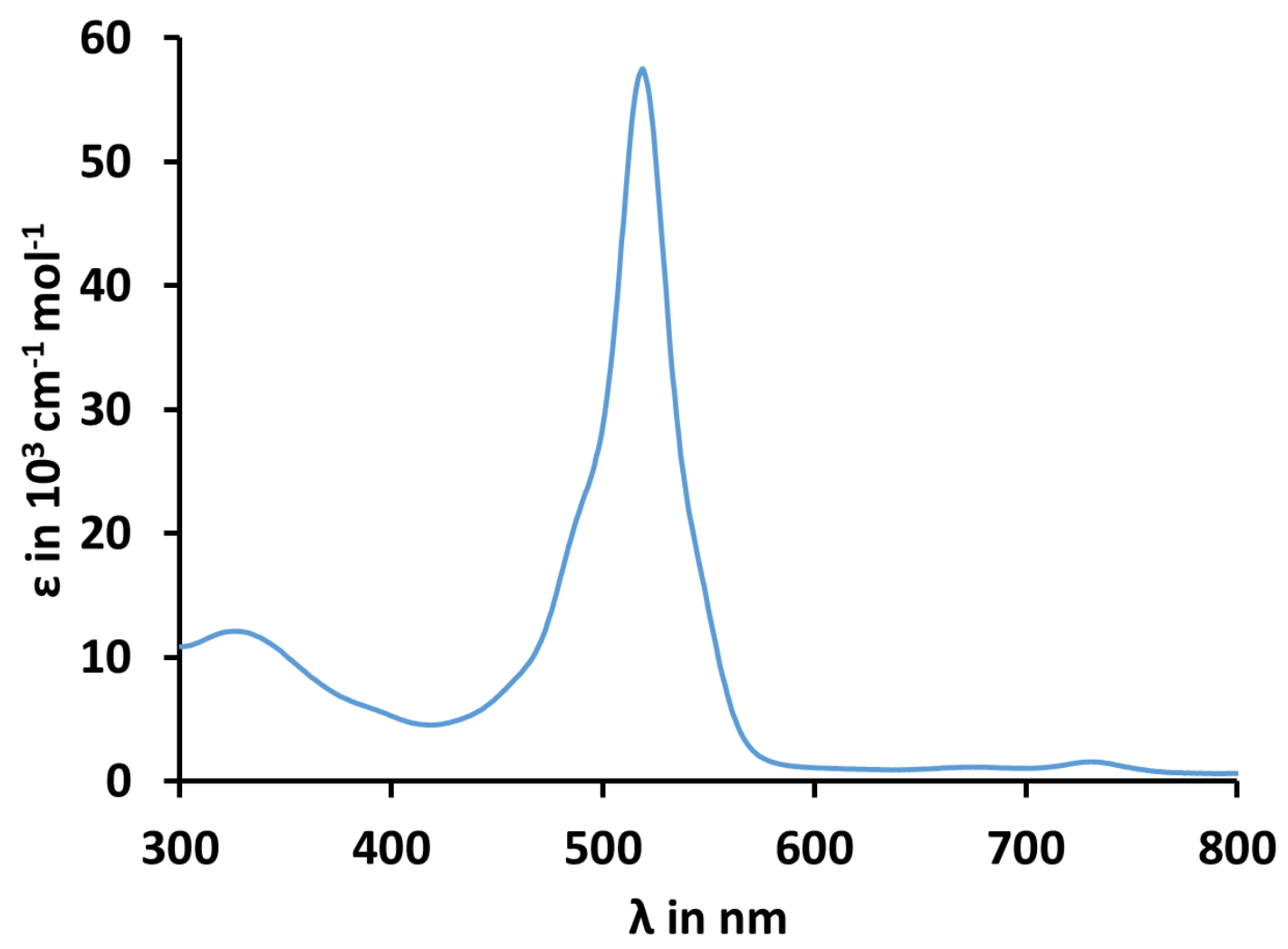

Figure S43: $\quad$ UV-Vis spectrum of complex 5 in DME at $298 \mathrm{~K}$.

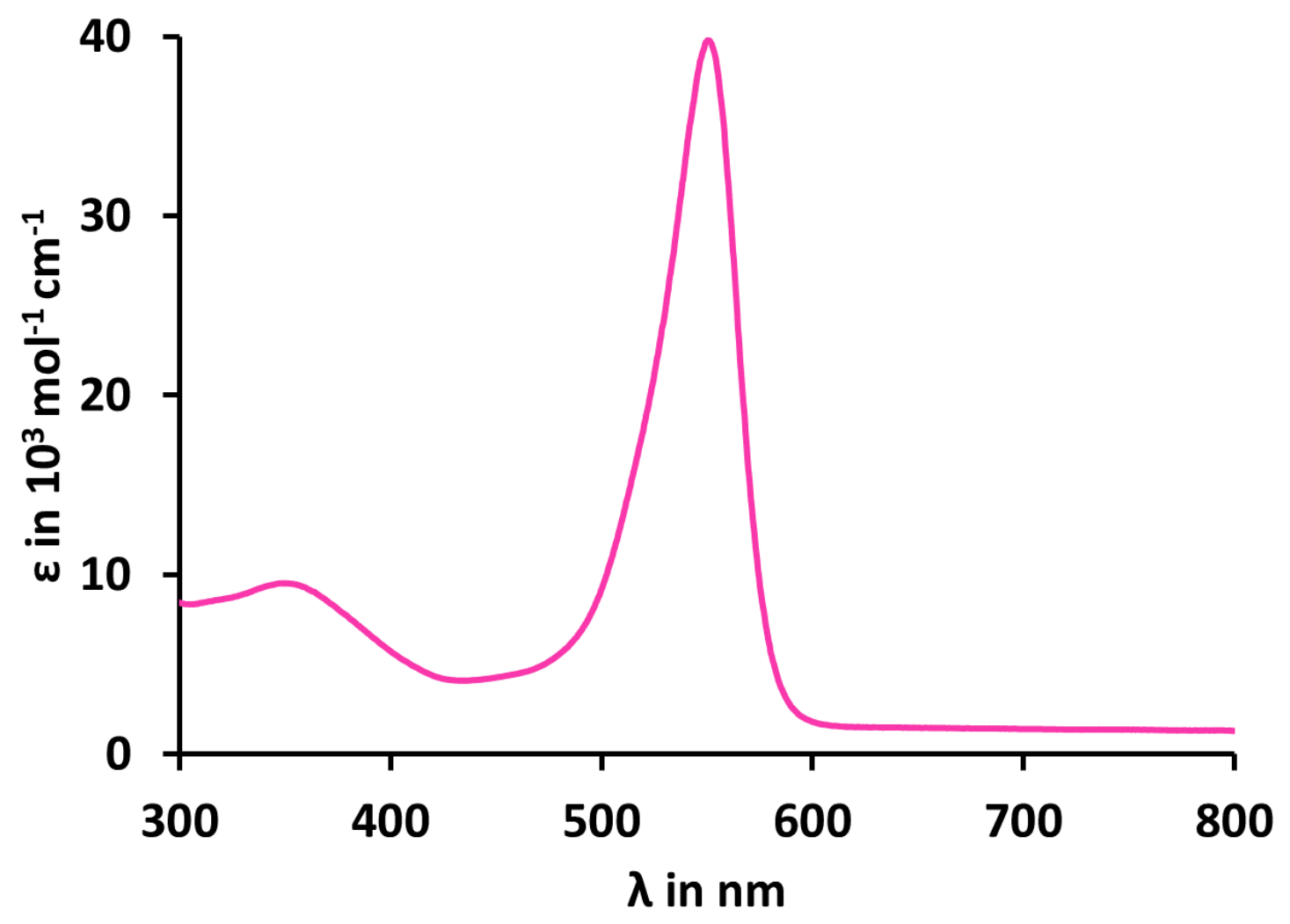

Figure S44: $\quad$ UV-Vis spectrum of complex 6 in DME at $298 \mathrm{~K}$. 


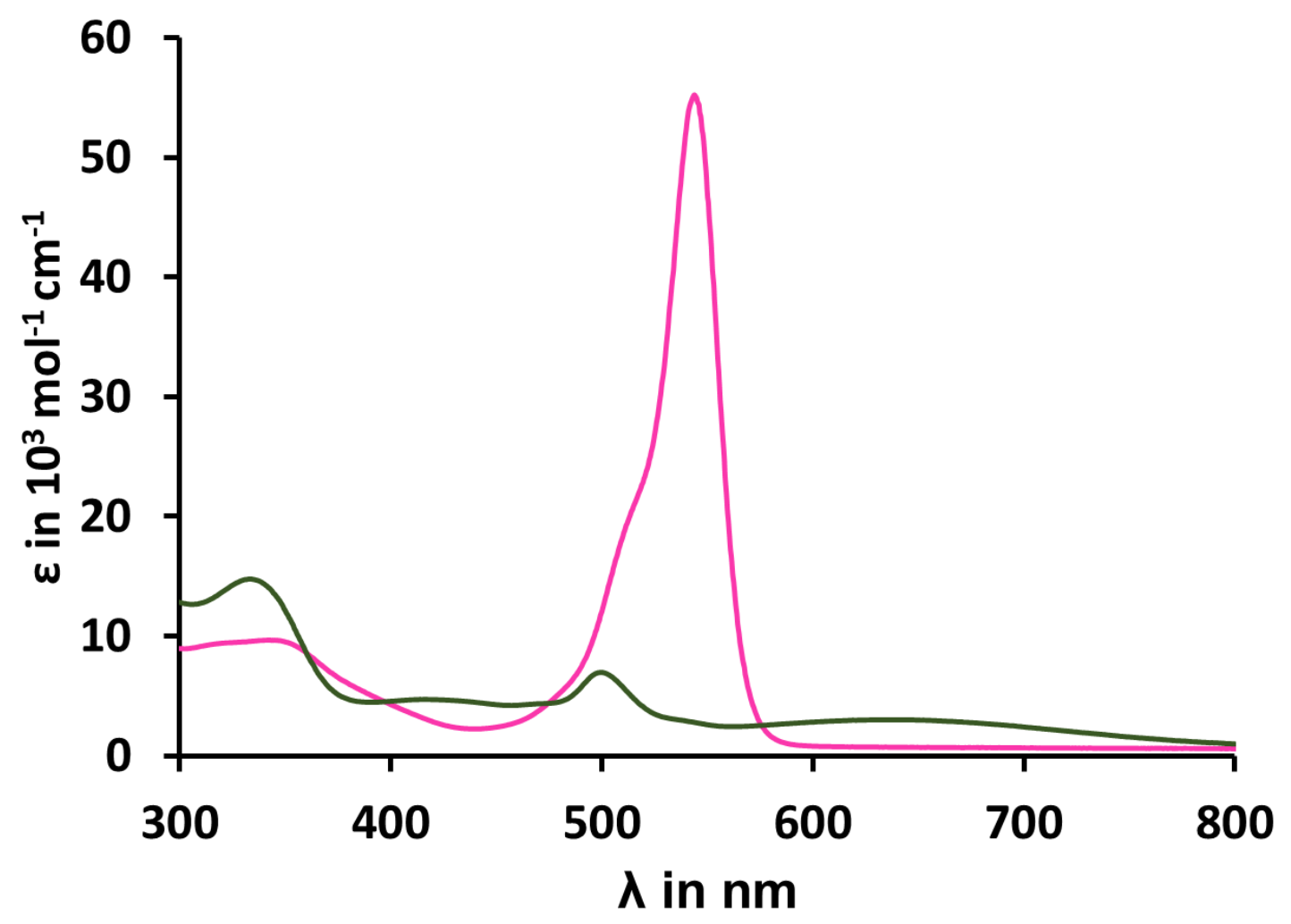

Figure S45: $\quad$ UV-Vis spectra of 1 (pink) and 7 (green) in DME at $298 \mathrm{~K}$.

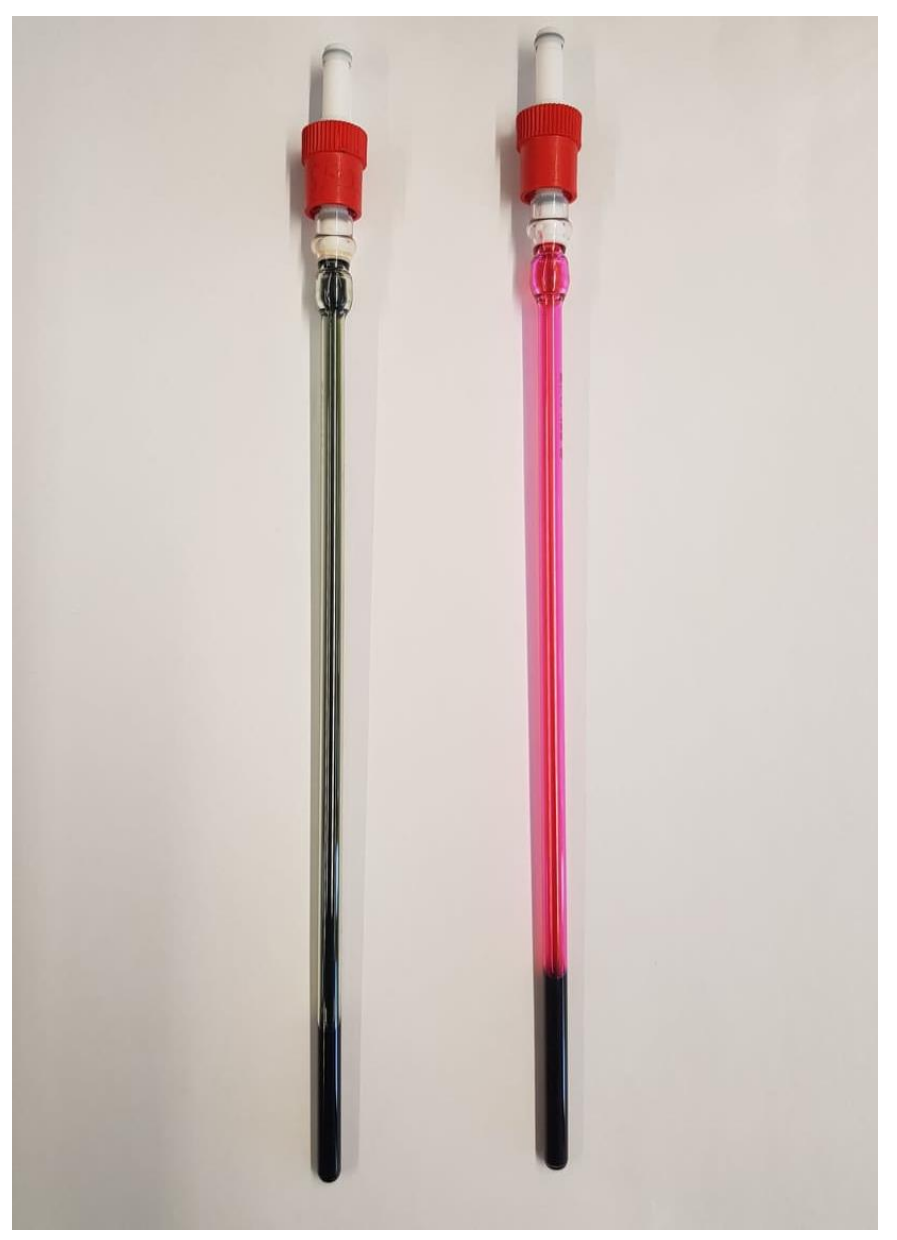

Figure S46: Comparison of colors between complex $\mathbf{7}$ (left ) and $\mathbf{1}$ (right) at room temperature. 


\section{Cyclic voltammetry}
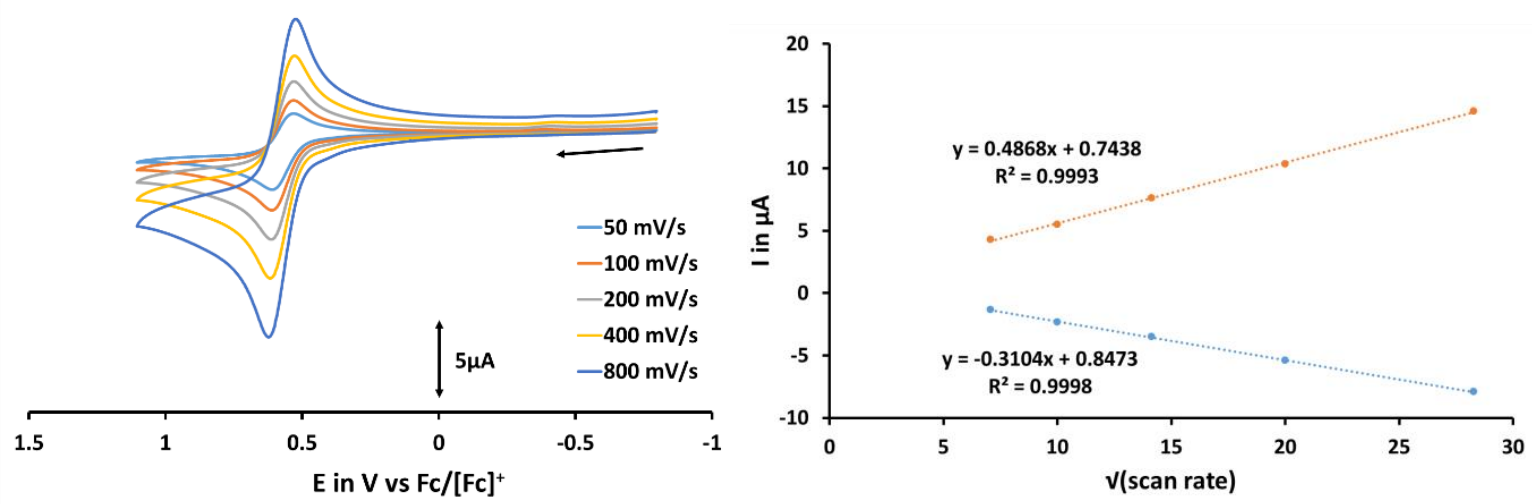

Figure S47: $\quad$ First oxidation of complex 1 measured at different scan rates (left) and reversibility plot (peak currencies vs (scan rate) ${ }^{0.5}$, right) at $298 \mathrm{~K}$. Electrolyte: $0.1 \mathrm{M} \mathrm{NBu}_{4} \mathrm{PF}_{6}$ in DCM; Complex concentration: $0.001 \mathrm{M}$.
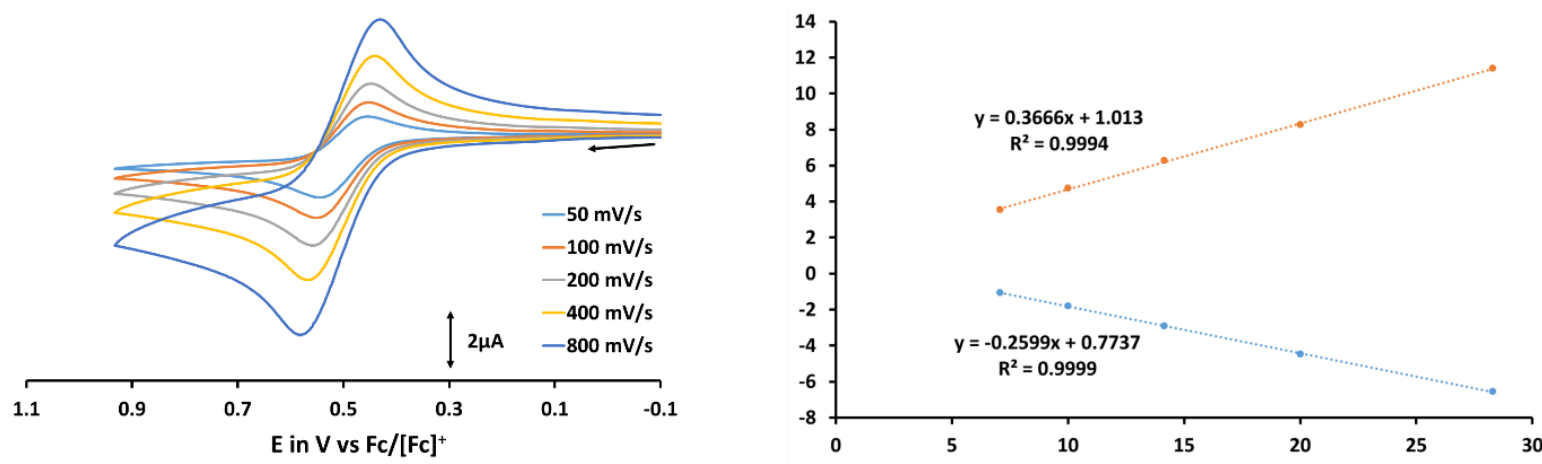

Figure S48: First oxidation of complex 3 measured at different scan rates (left) and reversibility plot (peak currencies vs (scan rate) ${ }^{0.5}$, right) at $298 \mathrm{~K}$. Electrolyte: $0.1 \mathrm{M} \mathrm{NBu}_{4} \mathrm{PF}_{6}$ in DCM; Complex concentration: $0.001 \mathrm{M}$. 


\section{Crystallographic details}

Table S1: Crystallographic details of complexes $\mathbf{1} \mathbf{- 7}$.

\begin{tabular}{|c|c|c|c|c|c|c|c|c|}
\hline & 1 & 2.pent & 2 & 3 & 4 & 5 & 6 & 7 \\
\hline Chemical formula & $\mathrm{C}_{44} \mathrm{H}_{55} \mathrm{~N}_{4} \mathrm{Cl}_{1} \mathrm{Mo}_{1}$ & $\begin{array}{l}\mathrm{C}_{54} \mathrm{H}_{59} \mathrm{~N}_{4} \mathrm{Cl}_{1} \mathrm{Mo}_{1} \\
\cdot \mathrm{C}_{5} \mathrm{H}_{12}\end{array}$ & $\mathrm{C}_{54} \mathrm{H}_{59} \mathrm{~N}_{4} \mathrm{Cl}_{1} \mathrm{Mo}_{1}$ & $\mathrm{C}_{34} \mathrm{H}_{51} \mathrm{~N}_{4} \mathrm{Cl}_{1} \mathrm{Mo}_{1}$ & $\mathrm{C}_{44} \mathrm{H}_{55} \mathrm{~N}_{4} \mathrm{Cl}_{1} \mathrm{Mo}_{1}$ & $\mathrm{C}_{44} \mathrm{H}_{55} \mathrm{~N}_{4} \mathrm{Mo}_{1}$ & $\begin{array}{l}\mathrm{C}_{44} \mathrm{H}_{55} \mathrm{~N}_{4} \mathrm{Mo}_{1} \\
\mathrm{O}_{3} \mathrm{~S}_{1} \mathrm{C}_{1} \mathrm{~F}_{3}\end{array}$ & $\mathrm{C}_{48} \mathrm{H}_{66} \mathrm{~N}_{4} \mathrm{Si}_{1} \mathrm{Mo}_{1}$ \\
\hline$M\left(\mathrm{~g} \cdot \mathrm{mol}^{-1}\right)$ & 771.31 & 967.58 & 985.44 & 647.17 & 771.31 & 735.86 & 884.93 & 823.7 \\
\hline Crystal system & Triclinic & Monoclinic & Monoclinic & Monoclinic & Triclinic & Triclinic & Monoclinic & Monoclinic \\
\hline Space group & $P-1$ & $P 2_{1} / c$ & $P 2_{1} / c$ & $P 2_{1} / n$ & $P-1$ & $P-1$ & $P 2_{1} / c$ & $P 2_{1} / c$ \\
\hline$a(\AA ̊)$ & $10.955(1)$ & $18.208(1)$ & $16.0501(5)$ & $14.190(2)$ & $11.2080(5)$ & $16.851(2)$ & $21.394(1)$ & $13.163(2)$ \\
\hline b (Å) & $12.798(1)$ & $14.925(1)$ & $14.4470(4)$ & $16.129(2)$ & $12.1211(6)$ & $16.913(2)$ & $13.177(1)$ & $28.389(5)$ \\
\hline$c(\AA ̊)$ & $18.567(1)$ & $19.224(1)$ & $20.6119(6)$ & $15.610(2)$ & $16.5441(8)$ & $29.047(4)$ & $17.820(1)$ & $13.357(2)$ \\
\hline$\left.\alpha 0^{\circ}\right)$ & $74.175(2)$ & 90 & 90 & 90 & $79.463(2)$ & $92.246(2)$ & 90 & 90 \\
\hline$\beta\left(^{\circ}\right)$ & $86.874(2)$ & $98.600(2)$ & $104.749(2)$ & $106.896(3)$ & $76.426(2)$ & $92.573(2)$ & $113.406(2)$ & $110.639(4)$ \\
\hline$y\left(^{\circ}\right)$ & $83.105(2)$ & 90 & 90 & 90 & $65.658(2)$ & $95.184(2)$ & 90 & 90 \\
\hline $\mathrm{V}\left(\AA^{3}\right)$ & $2485.7(4)$ & $5165.4(5)$ & $4621.9(2)$ & $3418.6(9)$ & 1981.1(2) & $8229(1)$ & $4610.4(7)$ & $4671(1)$ \\
\hline z & 2 & 4 & 4 & 4 & 2 & 8 & 4 & 4 \\
\hline Densitiy $\left(\mathrm{g} \mathrm{cm}^{-3}\right)$ & 1.031 & 1.244 & 1.287 & 1.257 & 1.293 & 1.188 & 1.275 & 1.170 \\
\hline$F(000)$ & 812 & 2048 & 1880 & 1368 & 812 & 3112 & 1848 & 1752 \\
\hline Radiation Type & $\mathrm{MoK}_{\alpha}$ & $\mathrm{MoK}_{\alpha}$ & $\mathrm{MoK}_{\alpha}$ & $\mathrm{MoK}_{\alpha}$ & $\mathrm{MoK}_{\alpha}$ & $\mathrm{MoK}_{\alpha}$ & $\mathrm{MoK}_{\alpha}$ & $\mathrm{MoK}_{\alpha}$ \\
\hline$\mu\left(\mathrm{mm}^{-1}\right)$ & 0.346 & 0.347 & 0.382 & 0.489 & 0.434 & 0.352 & 0.383 & 0.341 \\
\hline \multirow[t]{3}{*}{ Crystal size } & 0.25 & 0.45 & 0.25 & 0.15 & 0.12 & 0.25 & 0.20 & 0.15 \\
\hline & 0.22 & 0.42 & 0.24 & 0.14 & 0.10 & 0.22 & 0.18 & 0.12 \\
\hline & 0.17 & 0.38 & 0.21 & 0.11 & 0.07 & 0.20 & 0.16 & 0.11 \\
\hline Meas. Refl. & 27975 & 76745 & 105648 & 22396 & 45357 & 91527 & 45635 & 30896 \\
\hline Indep. Refl. & 9144 & 9078 & 8119 & 6310 & 7515 & 30446 & 8538 & 8562 \\
\hline Obsvd. $[I>2 \sigma(I)]$ & 7440 & 6279 & 6759 & 4842 & 5991 & 21463 & 4712 & 5696 \\
\hline $\mathrm{R}_{\text {int }}$ & 0.0496 & 0.1736 & 0.0832 & 0.0641 & 0.1233 & 0.0833 & 0.1675 & 0.1048 \\
\hline $\mathrm{R}\left[\mathrm{F}^{2}>2 \sigma\left(\mathrm{F}^{2}\right)\right]$ & 0.0368 & 0.0675 & 0.0348 & 0.0385 & 0.0655 & 0.1070 & 0.0438 & 0.0611 \\
\hline $\mathrm{WR}\left(\mathrm{F}^{2}\right)$ & 0.0932 & 0.1445 & 0.1101 & 0.0936 & 0.1294 & 0.2740 & 0.1257 & 0.1499 \\
\hline $\mathrm{s}$ & 1.022 & 1.157 & 1.197 & 1.010 & 1.156 & 1.090 & 0.823 & 1.017 \\
\hline$\Delta \rho_{\max }$ & 0.643 & 0.634 & 0.464 & 0.361 & 0.576 & 2.328 & 0.623 & 1.296 \\
\hline$\Delta \rho_{\min }$ & -0.585 & -0.465 & -0.855 & -0.359 & -0.772 & -1.558 & -0.772 & -0.36 \\
\hline CCDC & 1962501 & 1962505 & 1962506 & 1962504 & 1962502 & 1962503 & 1962508 & 1962507 \\
\hline
\end{tabular}


Table S2: Selected Bond lengths (in $\AA$ ) and angles (in ${ }^{\circ}$ ) of complexes 1 - 7.

\begin{tabular}{|c|c|c|c|c|c|c|c|c|}
\hline & 1 & 2 pent & 2 & 3 & 4 & 5 & 6 & 7 \\
\hline Mo1 - Cl1 / O100 & $2.408(1)$ & $2.388(1)$ & $2.380(1)$ & $2.385(1)$ & $2.410(1)$ & - & $2.120(3)$ & - \\
\hline Mo1-N1 & $2.191(2)$ & $2.240(4)$ & $2.142(2)$ & $2.164(2)$ & $2.267(4)$ & $2.16(1)$ & $2.149(3)$ & $1.978(4)$ \\
\hline Mo1 - N2 & $2.155(2)$ & $2.149(4)$ & $2.265(2)$ & $2.274(2)$ & $2.186(4)$ & $2.15(1)$ & $2.192(3)$ & $2.037(4)$ \\
\hline Mo1 - N40 & $1.742(2)$ & $1.764(4)$ & $1.753(2)$ & $1.728(2)$ & $1.745(4)$ & $1.73(1)$ & $1.723(3)$ & $1.736(4)$ \\
\hline Mo1 - N50 & $1.728(2)$ & $1.761(4)$ & $1.756(2)$ & $1.731(2)$ & $1.765(4)$ & $1.73(1)$ & $1.731(3)$ & $1.738(4)$ \\
\hline $\mathrm{C} 1-\mathrm{C} 2$ & $1.390(3)$ & $1.381(7)$ & $1.402(4)$ & $1.410(4)$ & $1.393(6)$ & $1.39(1)$ & $1.395(6)$ & $1.447(6)$ \\
\hline $\mathrm{C} 1-\mathrm{C} 3$ & $1.408(3)$ & $1.419(7)$ & $1.381(4)$ & $1.387(4)$ & $1.418(6)$ & $1.39(1)$ & $1.390(6)$ & $1.373(6)$ \\
\hline $\mathrm{C} 2-\mathrm{C} 4$ & $1.423(3)$ & $1.432(7)$ & $1.402(4)$ & $1.397(4)$ & $1.419(7)$ & $1.41(7)$ & $1.401(6)$ & $1.375(6)$ \\
\hline $\mathrm{C} 3-\mathrm{C} 5$ & $1.405(3)$ & $1.389(7)$ & $1.418(4)$ & $1.415(4)$ & $1.401(6)$ & $1.41(1)$ & $1.409(6)$ & $1.523(6)$ \\
\hline $\mathrm{C} 4-\mathrm{C} 6$ & $1.370(3)$ & $1.362(7)$ & $1.376(4)$ & $1.376(4)$ & $1.360(7)$ & $1.37(1)$ & $1.368(6)$ & $1.400(7)$ \\
\hline $\mathrm{C} 5-\mathrm{C} 7$ & $1.374(4)$ & $1-380(7)$ & $1.357(4)$ & $1.355(4)$ & $1.378(7)$ & $1.36(1)$ & $1.361(6)$ & $1.494(6)$ \\
\hline $\mathrm{C} 6-\mathrm{C} 8$ & $1.419(3)$ & $1.423(7)$ & $1.396(4)$ & $1.405(4)$ & $1.416(7)$ & $1.37(1)$ & $1.402(5)$ & $1.385(6)$ \\
\hline $\mathrm{C} 7-\mathrm{CP}$ & $1.404(3)$ & $1.405(7)$ & $1.426(4)$ & $1.420(4)$ & $1.400(6)$ & $1.41(1)$ & $1.407(6)$ & $1.329(6)$ \\
\hline $\mathrm{C} 8-\mathrm{N} 1$ & $1.349(3)$ & $1.346(6)$ & $1.376(3)$ & $1.361(3)$ & $1.337(6)$ & $1.34(1)$ & $1.354(5)$ & $1.377(6)$ \\
\hline $\mathrm{C} 9-\mathrm{N} 2$ & $1.353(3)$ & $1.365(6)$ & $1.347(3)$ & $1.341(3)$ & $1.372(6)$ & $1.34(1)$ & $1.366(5)$ & $1.426(6)$ \\
\hline $\mathrm{N} 1-\mathrm{C} 2$ & $1.402(3)$ & $1.397(6)$ & $1.403(3)$ & $1.405(4)$ & $1.416(6)$ & $1.41(1)$ & $1.415(5)$ & $1.418(6)$ \\
\hline $\mathrm{N} 2-\mathrm{C} 3$ & $1.404(3)$ & $1.415(6)$ & $1.415(4)$ & $1.411(3)$ & $1.409(6)$ & $1.41(1)$ & $1.402(5)$ & $1.426(6)$ \\
\hline N1 - Mo1 - N2 & 78.1(1) & $80.5(1)$ & $80.7(8)$ & 77..5(1) & 76.3(1) & $84.5(3)$ & 79.9(1) & $93.8(1)$ \\
\hline N40-Mo1-N50 & 111.5(1) & $105.6(2)$ & 107.9(1) & 107.3(1) & $108.7(2)$ & $112.4(4)$ & $110.7(2)$ & $114.0(2)$ \\
\hline Mo1 - N40-C40 & $169.8(2)$ & $169.8(4)$ & $148.5(2)$ & $171.6(2)$ & 168.9(3) & $172.1(8)$ & $176.7(3)$ & $174.4(4)$ \\
\hline Mo1 - N50 - C50 & $154.7(2)$ & $155.2(3)$ & $170.9(2)$ & $150.2(2)$ & $165.6(3)$ & $156.5(7)$ & $157.2(3)$ & 155.1(3) \\
\hline$\tau_{5}$ & 0.244 & 0.094 & - & 0.043 & 0.386 & - & 0.328 & - \\
\hline$\tau_{4}^{\prime}$ & - & - & - & - & - & 0.897 & - & 0.935 \\
\hline
\end{tabular}




\section{Computational Details}
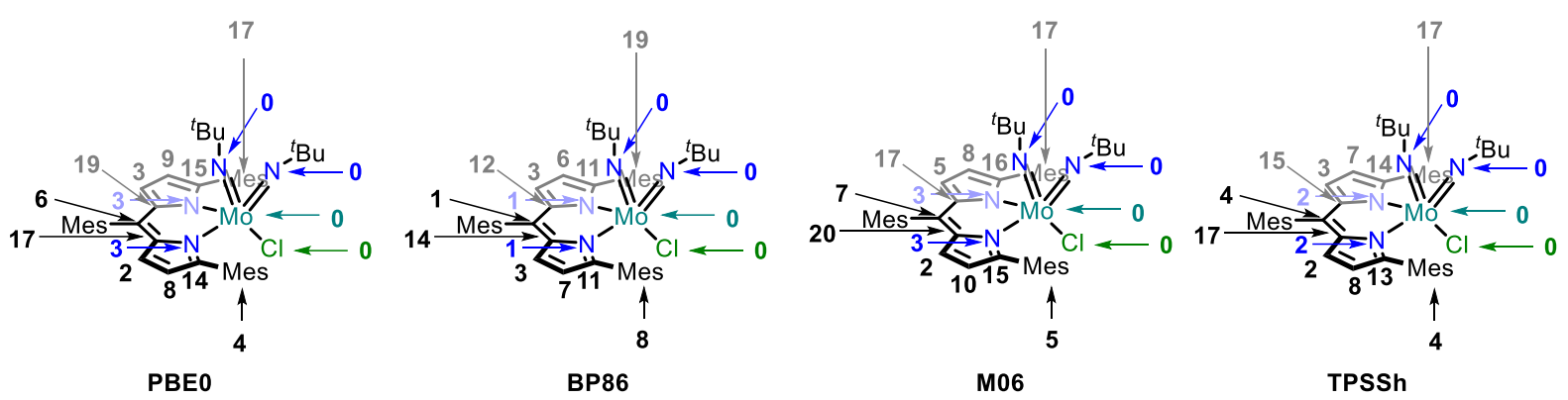

Figure S49: Löwdin spin densities (values given in \% a.u.) of $[\mathbf{1}]^{+}$as obtained by single-point calculations using various functionals (i.e., PBE0-D3BJ/def2-TZVPP//PBE0-D3BJ/def2SVP; BP86-D3BJ/def2-TZVPP//PBE0-D3BJ/def2-SVP; M06-D3ZERO/def2-TZVPP//PBE0D3BJ/def2-SVP; TPSSh-D3BJ/def2-TZVPP//PBE0-D3BJ/def2-SVP; M06-D3ZERO/def2TZVPP//PBE0-D3BJ/def2-SVP).

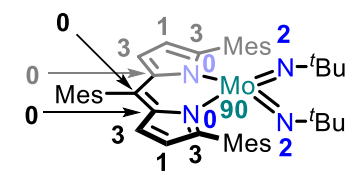

PBEO

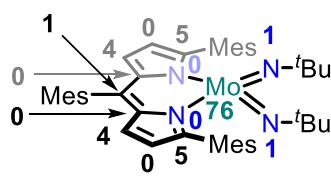

BP86

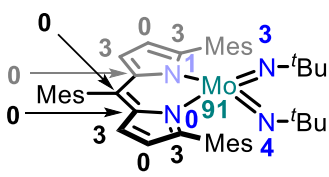

M06

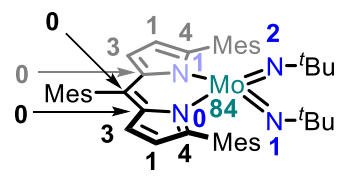

TPSSh

Figure 550: Löwdin spin densities (values given in \% a.u.) of $\mathbf{5}$ as obtained with various functionals (i.e., PBE0-D3BJ/def2-TZVPP//PBE0-D3BJ/def2-SVP; BP86-D3BJ/def2-TZVPP//PBE0D3BJ/def2-SVP; M06-D3ZERO/def2-TZVPP//PBE0-D3BJ/def2-SVP; TPSSh-D3BJ/def2TZVPP//PBE0-D3BJ/def2-SVP; M06-D3ZERO/def2-TZVPP//PBEO-D3BJ/def2-SVP).

Table S3: $\quad$ The SOMO - SOMO-1 energy difference $\Delta E$ is very small for [1 $]^{+}$and the DFT calculations show moderate spin contamination $\left(\left\langle S^{2}\right\rangle\right)$ in reference to the ideal value for a doublet $[S \cdot(S+1)=0.75]$.

\begin{tabular}{|l|l|l|}
\hline Functional & $\Delta E$ & $\left\langle\mathbf{S}^{2}\right\rangle$ \\
\hline PBE0//PBE0 & $0.19 \mathrm{eV}$ & 0.80 \\
\hline M06//PBEO & $0.27 \mathrm{eV}$ & 0.80 \\
\hline TPSSh//PBEO & $0.09 \mathrm{eV}$ & 0.78 \\
\hline BP//PBEO & $0.08 \mathrm{eV}$ & 0.76 \\
\hline
\end{tabular}



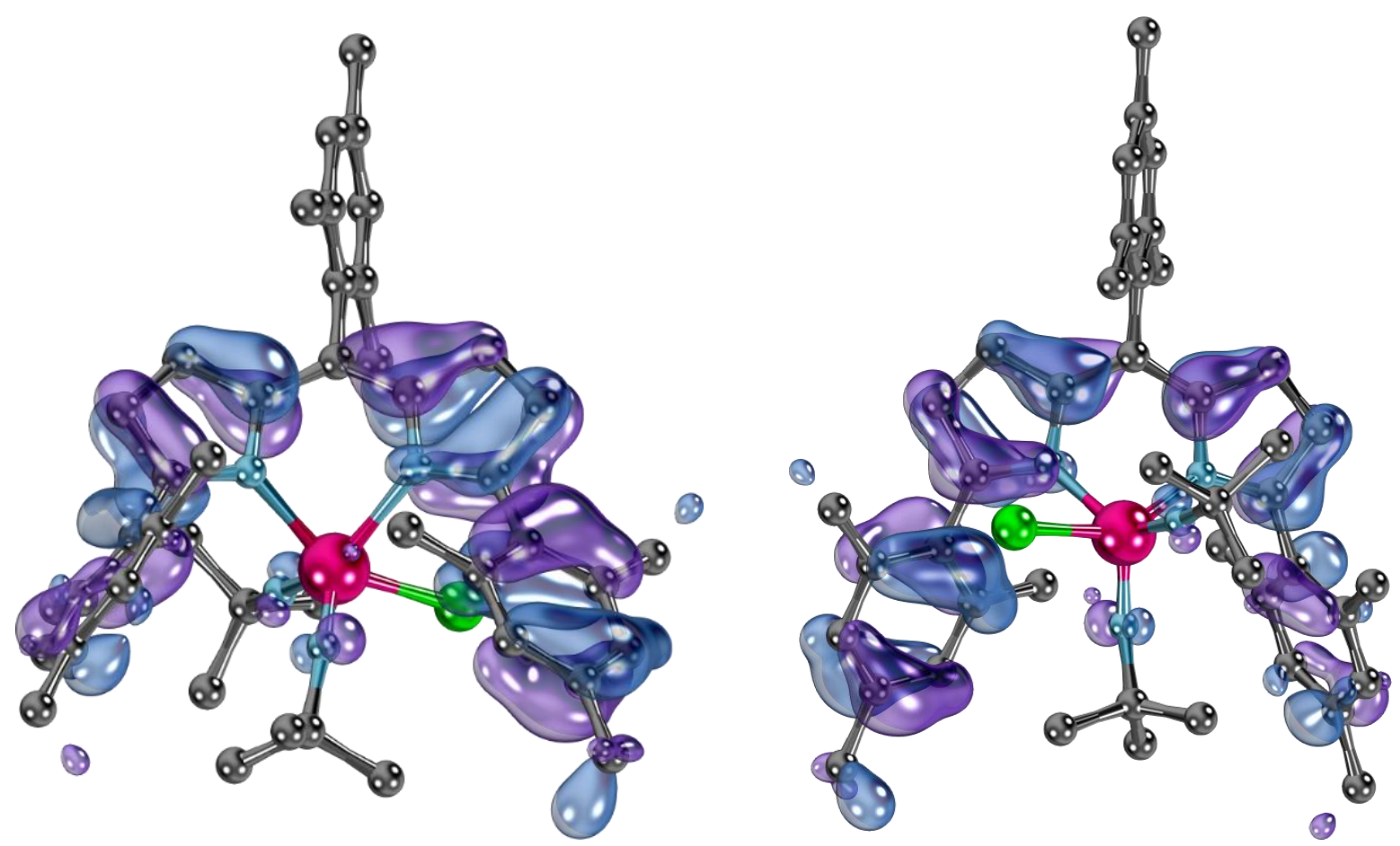

Figure S51: Singly occupied molecular orbital (SOMO) of [1] $]^{+}$as obtained from the PBEOD3BJ/def2-TZVPP//PBEO-D3BJ/def2-SVP level of theory.
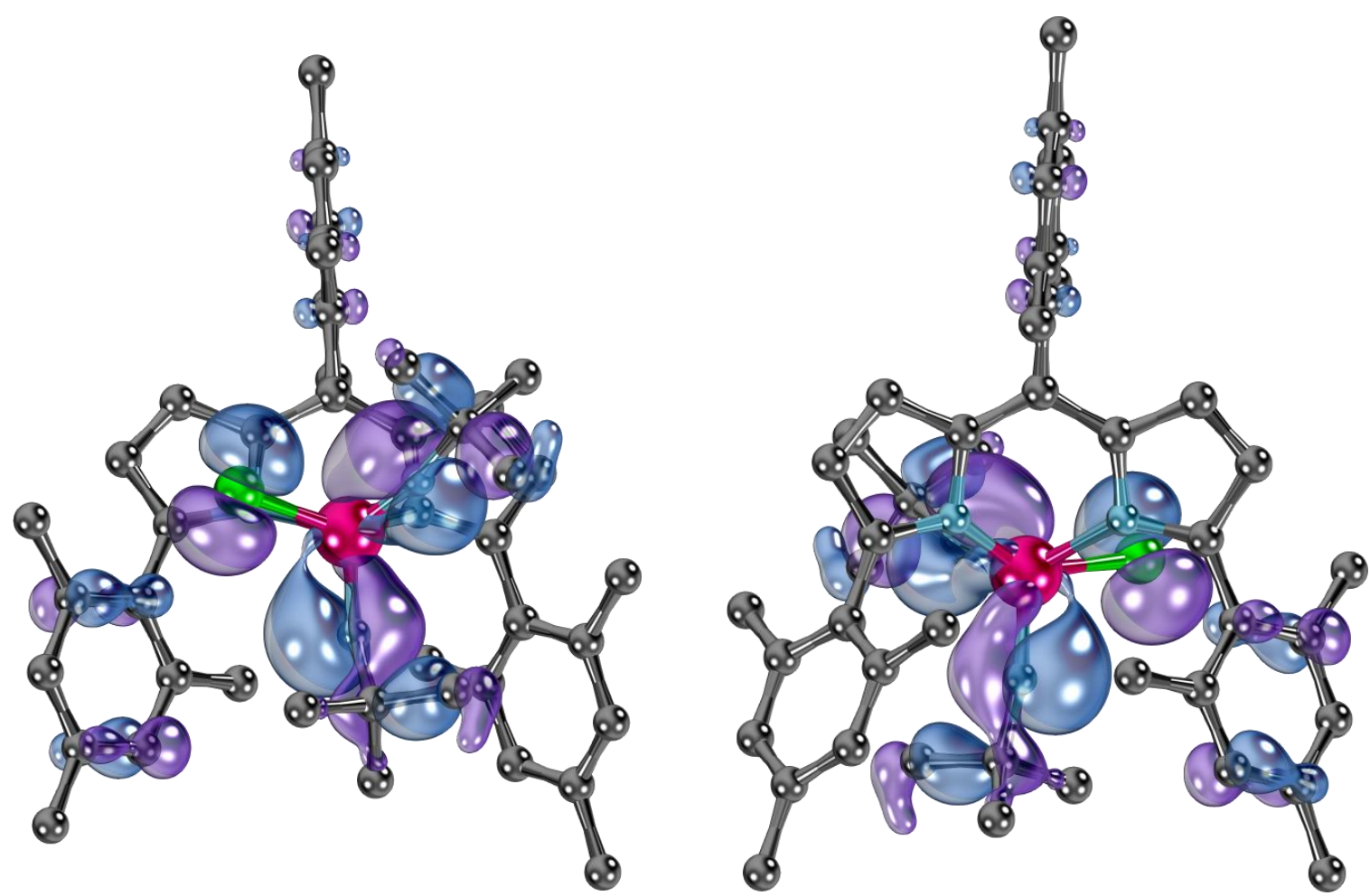

Figure S52: The highest doubly-occupied molecular orbital (i.e., SOMO-1) of [1] $]^{+}$is centered at the molybdenum-imido moieties and low in energy (PBEO-D3BJ/def2-TZVPP//PBEOD3BJ/def2-SVP: $\triangle E($ SOMO - SOMO-1) $=0.19 \mathrm{eV}$; vide supra $)$. 


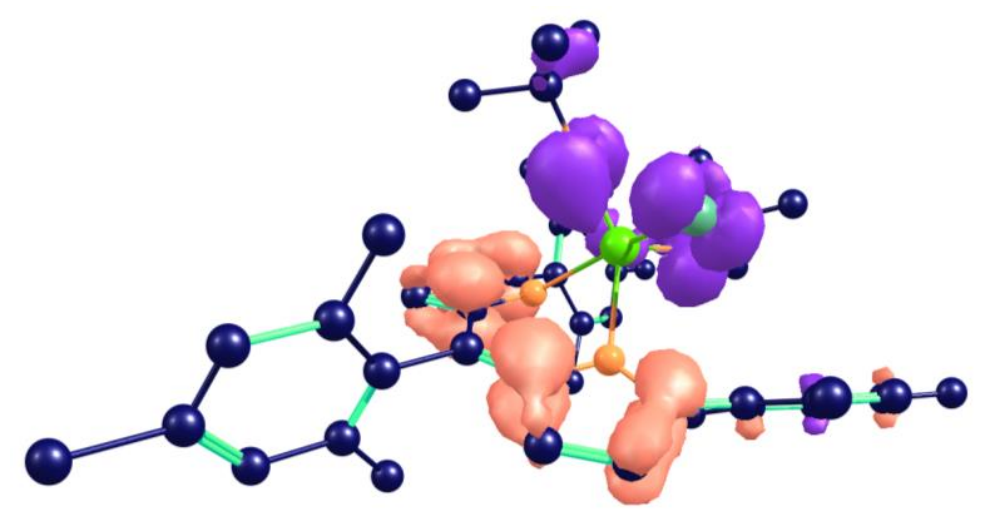

Figure S53: $\quad$ The $D_{0} \rightarrow D_{1}$ of $[1]^{+}$relates to the imido $\rightarrow$ DPM transition as is indicated by the difference density from the TDDFT calculations. Purple relates to the depletion, orange to gain of electron density.

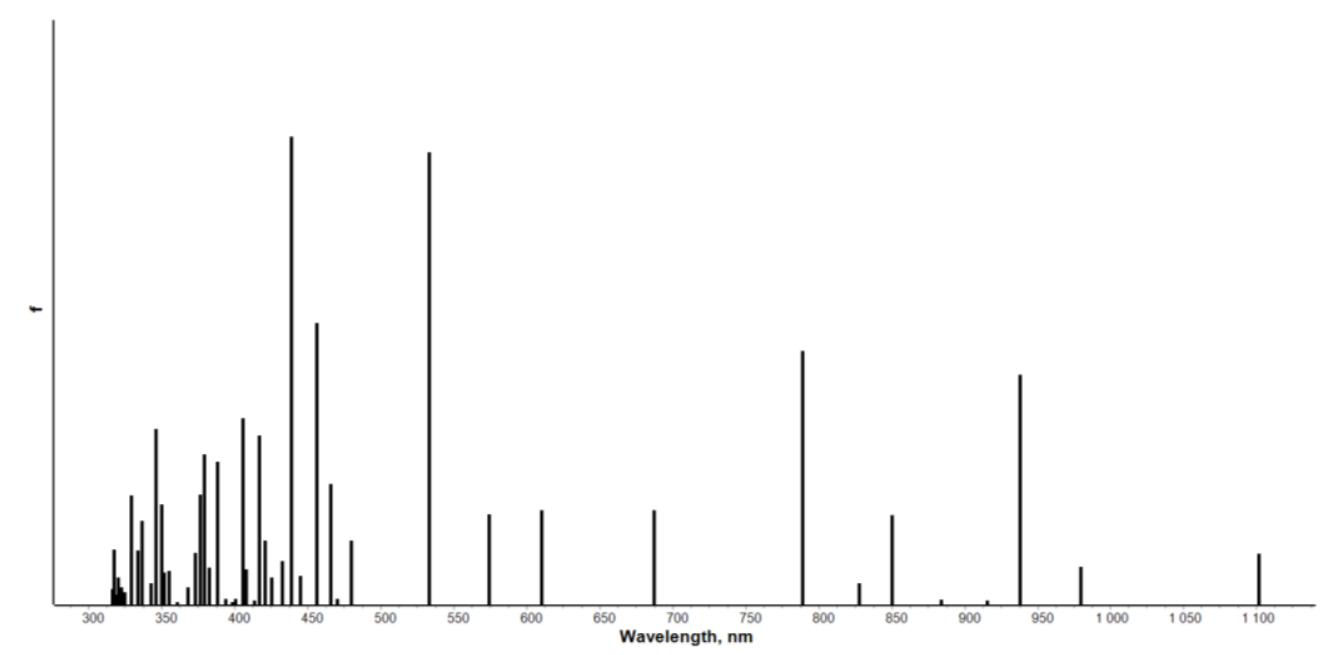

Figure S54: $\quad$ The TDDFT calculated UV-Vis spectrum of [1] ${ }^{+}$suggests an imido $\rightarrow$ DPM transition at $\lambda 1101 \mathrm{~nm}$ and further mesityl $\rightarrow$ DPM transitions between $\lambda=500$ and $1000 \mathrm{~nm}$.

ROOT O: $E=-2453.577991 \mathrm{Eh}$

0.82445 [ 0$]: 22222100000000$

$0.02479\left[\begin{array}{c}88]: 22220100002000 \\ 0.01498[\end{array}\right.$

0.01498 [ 848]: 22202100200000

0.01408 [ 16875]: 21212100110000

0.01306 [ 7235]: 22022100000020

0.00972 [ 44579]: 20222100020000

0.00874 [ 92758]: 12122100000110

0.00665 [ 8]: 22222000000001

0.00585 [217684] : 02222100000200

0.00438 [ 4]: 22222000010000

0.00337 [ 44581]: 20222100010100

0.00299 [ 87803]: 12222110000000

0.00299 [ 14]: 22221100001000

0.00259 [ 92733 ]: 12122110000010
ROOT 1: $E=-2453.524230 \mathrm{Eh}$

0.73220 [ 9]: 22221200000000

0.05564 [ 58]: 22220200001000

0.02900 [ 18]: 22221020000000

0.01347 [ 996]: 22201200200000

0.01266 [ 17023]: 21211200110000

0.01219 [ 210]: 22212200000000

0.01177 [ 7383]: 22021200000020

0.01030 [ 2277]: 22122200000000

0.00881 [ 44727]: 20221200020000

0.00839 [ 92906 ]: 12121200000110

0.00554 [217832]: 02221200000200

0.00356 [ 10]: 22221110000000

0.00303 [ 44729]: 20221200010100

0.00290 [ 23]: 22221010000100

0.00284 [ 87802 ]: 12222200000000

Figure S55: Configurations and weight of the $D_{0}$ ground state (left) and $D_{1}$ first excited state (right) of [1] $]^{+}$; energies relate to the NEVPT2 values. 


\section{Energies}

Table S4: $\quad$ Calculated energies of $[\mathbf{1}]^{+}$and $\mathbf{5}$.

\begin{tabular}{|c|c|c|c|c|c|c|c|}
\hline Name & NImag & E PBE0/SVP & G PBEO/SVP & $\begin{array}{l}\text { E PBE0/TZVPP// } \\
\text { PBEO/SVP }\end{array}$ & $\begin{array}{l}\text { E BP86/TZVPP// } \\
\text { PBE0/SVP }\end{array}$ & $\begin{array}{l}\text { E M06/TZVPP// } \\
\text { PBE0/SVP }\end{array}$ & $\begin{array}{l}\text { E M06/TZVPP// } \\
\text { PBE0/SVP }\end{array}$ \\
\hline & & {$[\mathrm{H}]$} & {$[\mathrm{H}]$} & {$[H]$} & {$[\mathrm{H}]$} & {$[H]$} & {$[\mathrm{H}]$} \\
\hline$[1]^{+}$ & 0 & -2453.404147 & -2452.59536 & -2455.57564 & -2458.33216 & -2456.42649 & -2458.26659 \\
\hline 5 & 0 & -1993.677032 & -1992.86897 & -1995.69378 & -1998.24217 & -1996.40949 & -1998.20897 \\
\hline
\end{tabular}

\section{XYZ Coordinates}

\section{Complex $[1]^{+}$}

$\begin{array}{lccc}\text { Mo } & 3.68469 & 8.93650 & 3.86657 \\ \mathrm{Cl} & 2.42178 & 9.22931 & 1.87846 \\ \mathrm{~N} & 3.04722 & 10.39279 & 4.52511 \\ \mathrm{~N} & 5.59065 & 9.16719 & 4.89100 \\ \mathrm{~N} & 2.88297 & 7.58802 & 4.56443 \\ \mathrm{~N} & 5.21014 & 8.27795 & 2.33909 \\ \mathrm{C} & 1.31382 & 11.58603 & 5.77273 \\ \mathrm{C} & 3.88130 & 5.19764 & 9.90528 \\ \mathrm{C} & 3.63917 & 12.51996 & 5.47164 \\ \mathrm{C} & 2.51647 & 11.69262 & 4.83352 \\ \mathrm{C} & 2.07952 & 12.33452 & 3.50940 \\ \mathrm{C} & 1.21786 & 7.83521 & 6.33838 \\ \mathrm{C} & 6.87277 & 6.48287 & 6.08305 \\ \mathrm{C} & 3.90679 & 9.95672 & 8.29061 \\ \mathrm{C} & 1.35408 & 3.06379 & 1.16112 \\ \mathrm{C} & 10.48030 & 14.00271 & 0.81490 \\ \mathrm{C} & 2.28079 & 5.61896 & 5.79873 \\ \mathrm{C} & 9.58754 & 9.55795 & 3.01012 \\ \mathrm{C} & 5.73698 & 12.60444 & 1.79228 \\ \mathrm{C} & 4.40992 & 6.24423 & 8.97339 \\ \mathrm{C} & 0.71888 & 6.69756 & 4.14533 \\ \mathrm{C} & 6.80980 & 10.20392 & 6.51970 \\ \mathrm{C} & 3.97867 & 7.57145 & 9.06664 \\ \mathrm{C} & 4.41855 & 8.55324 & 8.18119 \\ \mathrm{C} & 8.13198 & 13.24069 & 1.32865 \\ \mathrm{C} & 5.19900 & 5.42597 & 3.30816 \\ \mathrm{C} & 5.32827 & 5.92008 & 7.97120 \\ \mathrm{C} & 3.29098 & 4.32330 & 2.16356 \\ \mathrm{C} & 7.21099 & 12.32558 & 1.84188 \\ \mathrm{C} & 9.50765 & 12.99751 & 1.35453 \\ \mathrm{C} & 3.07789 & 7.23315 & -0.99809 \\ \mathrm{C} & 4.16967 & 5.39884 & 2.22017 \\ \mathrm{C} & 6.71979 & 10.13893 & 2.92728 \\ \mathrm{C} & 9.07478 & 10.85145 & 2.44274 \\ \mathrm{C} & 3.18074 & 6.24176 & 0.12251\end{array}$




\begin{tabular}{|c|c|c|c|}
\hline C & 7.19528 & 10.76486 & 5.33616 \\
\hline C & 7.69566 & 11.13214 & 2.40601 \\
\hline$C$ & 2.32965 & 4.19672 & 1.15388 \\
\hline C & 5.78935 & 6.86279 & 7.05136 \\
\hline C & 2.30107 & 5.16605 & 0.14487 \\
\hline C & 5.30157 & 8.18597 & 7.14535 \\
\hline C & 5.82770 & 9.18513 & 6.20717 \\
\hline C & 9.95569 & 11.79597 & 1.91294 \\
\hline C & 4.98793 & 7.53272 & 1.23685 \\
\hline C & 4.10385 & 6.38705 & 1.19792 \\
\hline C & 1.77419 & 6.94691 & 5.22996 \\
\hline C & 5.82142 & 8.01399 & 0.14707 \\
\hline C & 6.46340 & 10.07878 & 4.30031 \\
\hline C & 6.16892 & 9.20883 & 2.01654 \\
\hline C & 6.56376 & 9.04580 & 0.63760 \\
\hline $\mathrm{H}$ & 1.58101 & 11.10348 & 6.72238 \\
\hline $\mathrm{H}$ & 0.92401 & 12.58861 & 6.00135 \\
\hline $\mathrm{H}$ & 0.50841 & 11.00497 & 5.30117 \\
\hline $\mathrm{H}$ & 3.65887 & 5.61355 & 10.89776 \\
\hline $\mathrm{H}$ & 2.94086 & 4.77653 & 9.51196 \\
\hline $\mathrm{H}$ & 4.58856 & 4.36549 & 10.02582 \\
\hline $\mathrm{H}$ & 3.27923 & 13.53590 & 5.68871 \\
\hline $\mathrm{H}$ & 3.98546 & 12.06791 & 6.40989 \\
\hline $\mathrm{H}$ & 4.49817 & 12.59393 & 4.78947 \\
\hline $\mathrm{H}$ & 1.32130 & 11.71835 & 3.00821 \\
\hline $\mathrm{H}$ & 1.65527 & 13.32998 & 3.70722 \\
\hline $\mathrm{H}$ & 2.93267 & 12.44371 & 2.82627 \\
\hline $\mathrm{H}$ & 0.34175 & 7.35695 & 6.80105 \\
\hline $\mathrm{H}$ & 1.97525 & 8.00014 & 7.11688 \\
\hline $\mathrm{H}$ & 0.90885 & 8.80696 & 5.93335 \\
\hline $\mathrm{H}$ & 7.85808 & 6.76572 & 6.49046 \\
\hline $\mathrm{H}$ & 6.88932 & 5.39823 & 5.91460 \\
\hline $\mathrm{H}$ & 6.76685 & 6.98145 & 5.11279 \\
\hline $\mathrm{H}$ & 3.29563 & 10.09095 & 9.19282 \\
\hline $\mathrm{H}$ & 4.72476 & 10.69286 & 8.32387 \\
\hline $\mathrm{H}$ & 3.28478 & 10.20389 & 7.41877 \\
\hline $\mathrm{H}$ & 1.80575 & 2.14348 & 1.55761 \\
\hline $\mathrm{H}$ & 0.49390 & 3.30652 & 1.80808 \\
\hline $\mathrm{H}$ & 0.96030 & 2.85747 & 0.15679 \\
\hline $\mathrm{H}$ & 10.89853 & 14.61523 & 1.63047 \\
\hline $\mathrm{H}$ & 11.32702 & 13.51492 & 0.31102 \\
\hline $\mathrm{H}$ & 10.00077 & 14.68785 & 0.10194 \\
\hline $\mathrm{H}$ & 3.09461 & 5.78885 & 6.51712 \\
\hline $\mathrm{H}$ & 1.45844 & 5.09844 & 6.31246 \\
\hline $\mathrm{H}$ & 2.64939 & 4.97100 & 4.99109 \\
\hline $\mathrm{H}$ & 9.32284 & 9.44150 & 4.07345 \\
\hline $\mathrm{H}$ & 9.16112 & 8.68993 & 2.48183 \\
\hline $\mathrm{H}$ & 10.68108 & 9.49630 & 2.92987 \\
\hline $\mathrm{H}$ & 5.19578 & 11.85184 & 1.19610 \\
\hline
\end{tabular}




$\begin{array}{cccc}\mathrm{H} & 5.29404 & 12.58363 & 2.79900 \\ \mathrm{H} & 5.53129 & 13.59006 & 1.35483 \\ \mathrm{H} & -0.13778 & 6.16036 & 4.57823 \\ \mathrm{H} & 0.36603 & 7.64898 & 3.72299 \\ \mathrm{H} & 1.14192 & 6.09660 & 3.32737 \\ \mathrm{H} & 7.19610 & 10.40657 & 7.51675 \\ \mathrm{H} & 3.27231 & 7.85124 & 9.85340 \\ \mathrm{H} & 7.76038 & 14.17046 & 0.88942 \\ \mathrm{H} & 4.84736 & 6.03404 & 4.15320 \\ \mathrm{H} & 5.38390 & 4.41028 & 3.68262 \\ \mathrm{H} & 6.15524 & 5.84810 & 2.97294 \\ \mathrm{H} & 5.71071 & 4.89776 & 7.90794 \\ \mathrm{H} & 3.36866 & 3.54172 & 2.92490 \\ \mathrm{H} & 3.18045 & 8.26787 & -0.64585 \\ \mathrm{H} & 3.84678 & 7.04987 & -1.76676 \\ \mathrm{H} & 2.10440 & 7.14007 & -1.49778 \\ \mathrm{H} & 7.95510 & 11.52365 & 5.15607 \\ \mathrm{H} & 1.55941 & 5.07862 & -0.65381 \\ \mathrm{H} & 11.02841 & 11.58208 & 1.92947 \\ \mathrm{H} & 5.88100 & 7.56466 & -0.84066 \\ \mathrm{H} & 7.33582 & 9.62647 & 0.13690\end{array}$

\section{Complex 5}

$\begin{array}{llll}\text { Mo } & -2.14262 & 4.04279 & 3.88923 \\ \text { N } & -4.03024 & 4.96794 & 3.56535 \\ \text { N } & -2.06639 & 2.35636 & 3.45349 \\ \text { N } & -1.43489 & 5.41002 & 2.41061 \\ \text { C } & -4.16222 & 6.33243 & 3.32023 \\ \text { C } & -1.91908 & 6.70952 & 2.28991 \\ \text { C } & -6.16736 & 5.57721 & 4.01523 \\ \text { H } & -7.21432 & 5.47878 & 4.29496 \\ \text { C } & -5.50932 & 3.09799 & 4.28878 \\ \text { C } & -5.23001 & 4.51402 & 3.97280 \\ \text { C } & -5.49582 & 6.72165 & 3.62032 \\ \text { H } & -5.88905 & 7.73144 & 3.51744 \\ \text { C } & -4.44929 & 10.39860 & 1.31059 \\ \text { H } & -4.96193 & 10.75281 & 0.41122 \\ \text { C } & 0.52017 & 4.10835 & 1.60105 \\ \text { C } & -5.69047 & 2.17364 & 3.24403 \\ \text { C } & -3.16971 & 7.14383 & 2.75251 \\ \text { N } & -1.54164 & 4.40906 & 5.48074 \\ \text { C } & -3.49598 & 8.58863 & 2.58306 \\ \text { C } & -0.27435 & 5.34700 & 1.73187 \\ \text { C } & 0.09090 & 3.09205 & 0.72537 \\ \text { C } & -0.99366 & 7.47059 & 1.52472 \\ \text { H } & -1.11380 & 8.52038 & 1.26380 \\ \text { C } & -5.66979 & 2.70121 & 5.62965 \\ \text { C } & -4.15166 & 9.03888 & 1.42325\end{array}$




\begin{tabular}{|c|c|c|c|}
\hline C & -2.46191 & 8.98996 & 4.85005 \\
\hline $\mathrm{H}$ & -3.08680 & 8.25741 & 5.38490 \\
\hline $\mathrm{H}$ & -2.23421 & 9.81711 & 5.53548 \\
\hline $\mathrm{H}$ & -1.52002 & 8.47504 & 4.60473 \\
\hline C & -3.14894 & 9.48305 & 3.60925 \\
\hline C & -4.11358 & 11.31384 & 2.31240 \\
\hline C & -6.06989 & 0.86739 & 3.56316 \\
\hline $\mathrm{H}$ & -6.22563 & 0.15064 & 2.75105 \\
\hline C & -5.46287 & 2.57313 & 1.81629 \\
\hline $\mathrm{H}$ & -5.95010 & 3.53071 & 1.57873 \\
\hline $\mathrm{H}$ & -5.84063 & 1.80872 & 1.12364 \\
\hline $\mathrm{H}$ & -4.38502 & 2.70514 & 1.62831 \\
\hline C & -6.03810 & 1.38397 & 5.90393 \\
\hline $\mathrm{H}$ & -6.15616 & 1.07483 & 6.94687 \\
\hline C & 0.89132 & 1.95864 & 0.57072 \\
\hline $\mathrm{H}$ & 0.56441 & 1.17102 & -0.11517 \\
\hline C & 0.02968 & 6.61028 & 1.16429 \\
\hline $\mathrm{H}$ & 0.90264 & 6.82788 & 0.55171 \\
\hline C & -1.75618 & 0.96611 & 3.66235 \\
\hline C & -6.25432 & 0.45328 & 4.88415 \\
\hline C & -3.46630 & 10.83484 & 3.45316 \\
\hline $\mathrm{H}$ & -3.20026 & 11.53412 & 4.25149 \\
\hline C & -4.52459 & 8.07661 & 0.33317 \\
\hline $\mathrm{H}$ & -5.18794 & 7.28289 & 0.71111 \\
\hline $\mathrm{H}$ & -3.63269 & 7.57448 & -0.07286 \\
\hline $\mathrm{H}$ & -5.03556 & 8.58858 & -0.49360 \\
\hline C & 1.72753 & 3.97604 & 2.31057 \\
\hline C & -5.41754 & 3.67740 & 6.74147 \\
\hline $\mathrm{H}$ & -4.42160 & 4.13361 & 6.63102 \\
\hline $\mathrm{H}$ & -5.46662 & 3.18438 & 7.72230 \\
\hline $\mathrm{H}$ & -6.14575 & 4.50319 & 6.73450 \\
\hline C & -4.42021 & 12.77471 & 2.15311 \\
\hline $\mathrm{H}$ & -3.68065 & 13.26515 & 1.49805 \\
\hline $\mathrm{H}$ & -4.40371 & 13.29752 & 3.11982 \\
\hline $\mathrm{H}$ & -5.40923 & 12.93072 & 1.69659 \\
\hline C & 2.08889 & 1.79734 & 1.27305 \\
\hline C & 2.48858 & 2.81766 & 2.13812 \\
\hline $\mathrm{H}$ & 3.41966 & 2.70936 & 2.70171 \\
\hline C & -1.03958 & 4.53212 & 6.81753 \\
\hline C & -6.62222 & -0.96518 & 5.20818 \\
\hline $\mathrm{H}$ & -7.06769 & -1.47588 & 4.34248 \\
\hline $\mathrm{H}$ & -7.33622 & -1.01612 & 6.04408 \\
\hline $\mathrm{H}$ & -5.72932 & -1.53997 & 5.50598 \\
\hline C & -1.21110 & 3.20768 & -0.01005 \\
\hline $\mathrm{H}$ & -2.04999 & 3.01293 & 0.67812 \\
\hline $\mathrm{H}$ & -1.26965 & 2.48023 & -0.83132 \\
\hline $\mathrm{H}$ & -1.35772 & 4.21652 & -0.42294 \\
\hline C & -2.57569 & 0.44178 & 4.84388 \\
\hline $\mathrm{H}$ & -2.32748 & 1.00397 & 5.75565 \\
\hline
\end{tabular}




$\begin{array}{lrrl}\text { H } & -2.36333 & -0.62526 & 5.01884 \\ \text { H } & -3.64967 & 0.56146 & 4.65153 \\ \text { C } & -2.12043 & 0.19739 & 2.38734 \\ \text { H } & -3.18933 & 0.32615 & 2.15981 \\ \text { H } & -1.91731 & -0.87846 & 2.50607 \\ \text { H } & -1.53437 & 0.57163 & 1.53523 \\ \text { C } & -0.26023 & 0.83397 & 3.95892 \\ \text { H } & 0.33320 & 1.27848 & 3.14921 \\ \text { H } & 0.02308 & -0.22453 & 4.07138 \\ \text { H } & -0.01344 & 1.36479 & 4.89086 \\ \text { C } & 2.18124 & 5.05588 & 3.24915 \\ \text { H } & 2.50177 & 5.95737 & 2.70304 \\ \text { H } & 3.02511 & 4.71760 & 3.86630 \\ \text { H } & 1.36541 & 5.36231 & 3.91940 \\ \text { C } & 2.88877 & 0.53470 & 1.14395 \\ \text { H } & 2.49324 & -0.24296 & 1.81932 \\ \text { H } & 3.94445 & 0.69300 & 1.40747 \\ \text { H } & 2.84430 & 0.13017 & 0.12207 \\ \text { C } & -1.35976 & 3.25432 & 7.59954 \\ \text { H } & -2.44375 & 3.06752 & 7.60004 \\ \text { H } & -1.01505 & 3.33325 & 8.64226 \\ \text { H } & -0.86695 & 2.38814 & 7.13335 \\ \text { C } & 0.47670 & 4.73827 & 6.75537 \\ \text { H } & 0.95510 & 3.89757 & 6.23139 \\ \text { H } & 0.90382 & 4.81010 & 7.76776 \\ \text { H } & 0.71747 & 5.66340 & 6.21066 \\ \mathrm{C} & -1.70634 & 5.74137 & 7.48355 \\ \text { H } & -1.48929 & 6.65560 & 6.91179 \\ \text { H } & -1.34137 & 5.87652 & 8.51391 \\ \text { H } & -2.79801 & 5.61094 & 7.51402\end{array}$




\section{Literature}

A. B. Scharf and T. A. Betley, Inorg. Chem., 2011, 50, 6837-6845.

E. R. King and T. A. Betley, Inorg. Chem., 2009, 48, 2361-2363.

E. R. King, E. T. Hennessy and T. A. Betley, J. Am. Chem. Soc., 2011, 133, 4917-4923.

K. A. Rufanov, D. N. Zarubin, N. A. Ustynyuk, D. N. Gourevitch, J. Sundermeyer, A. V. Churakov and J. A.K. Howard, Polyhedron, 2001, 20, 379-385.

a) H. H. Fox, K. B. Yap, J. Robbins, S. Cai and R. R. Schrock, Inorg. Chem., 1992, 31, 2287-2289;

b) P. W. Dyer, V. C. Gibson, J. A. K. Howard, B. Whittle and C. Wilson, J. Chem. Soc., Chem. Commun., 1992, 1666. c) U. Radius, J. Sundermeyer, H. Pritzkow, Chem. Ber. 1994, 127, 1827-1835.

a) D. E. Bergbreiter and J. M. Killough, J. Am. Chem. Soc., 1978, 100, 2126-2134; b) J.-M. Lalancette, G. Rollin and P. Dumas, Can. J. Chem., 1972, 50, 3058-3062.

H. J. Shine, B.-J. Zhao, J. N. Marx, T. Ould-Ely and K. H. Whitmire, J. Org. Chem., 2004, 69, 9255-9261.

I. Krossing, Chem. Eur. J., 2001, 7, 490-502.

a) F. Neese, WIREs Comput Mol Sci, 2012, 2, 73-78; b) F. Neese, WIREs Comput Mol Sci, 2018, 8, 33.

a) S. Grimme, J. Antony, S. Ehrlich and H. Krieg, J. Chem. Phys., 2010, 132, 154104; b) S. Grimme, S. Ehrlich and L. Goerigk, J. Comp. Chem., 2011, 32, 1456-1465; c) Perdew, Burke and Ernzerhof, Phys. Rev. Lett., 1996, 77, 3865-3868; d) F. Weigend and R. Ahlrichs, Phys. Chem. Chem. Phys., 2005, 7, 3297-3305; e) C. Adamo and V. Barone, J. Chem. Phys., 1999, 110, 6158-6170.

11 D. Andrae, U. Huermann, M. Dolg, H. Stoll and H. Preu, Theoret. Chim. Acta, 1990, 77, 123141.

a) F. Neese, F. Wennmohs, A. Hansen and U. Becker, Chemical Physics, 2009, 356, 98-109;

b) F. Weigend, Phys. Chem. Chem. Phys., 2006, 8, 1057-1065.

G. Knizia, J. Chem. Theory. Comp., 2013, 9, 4834-4843.

Sheldrick, G. M. Crystal structure refinement with SHELXL. Acta cryst. C 2015, 71, 3-8.

Dolomanov, O. V.; Bourhis, L. J.; Gildea, R. J.; Howard, J. A. K.; Puschmann, H. OLEX2 : a complete structure solution, refinement and analysis program. J Appl Crystallogr 2009, 42, 339-341.

van der Sluis, P.; Spek, A. L. BYPASS: an effective method for the refinement of crystal structures containing disordered solvent regions. Acta Cryst. A 1990, 46, 194-201. 